Universidad NACIONAL dE LA PLATA

FACULTAD DE HumANIDADES Y CIENCIAS DE LA EDUCACIÓN

SECRETARÍA DE Posgrado

\title{
Eficacia de la Actividad Física en la reducción del riesgo de enfermedades cardiovasculares, Un Meta-análisis
}

Christian Martín García

Tesis para optar por el grado de Magister en Deporte Director Dr. Sebastian Varela, UNLP

La Plata, 12 de Septiembre de 2014. 


\section{AGRADECIMIENTOS}

En primer lugar, es muy importante para mi mencionar que este trabajo no hubiera sido posible sin el apoyo de muchas personas. Quisiera agradecerles brevemente:

A Verónica, mi compañera de la vida, por todo el apoyo que me brindó, el enseñarme a no bajar los brazos y a superar las frustraciones y obstáculos, tan comunes en el camino del investigador.

A mi padre, por sus sabias enseñanzas y por haberme dado siempre todo lo mejor de sí.

A mi director, Sebastian Varela, por su apoyo incondicional, sus aportes y su invalorable predisposición en todo momento, acompañándome a lo largo de este camino.

A Adrian Casas, por todos sus aportes y recomendaciones profesionales en la temática.

A Marcelo Giles, por todo el apoyo institucional que he recibido a lo largo de esta hermosa carrera.

Con especial dedicación a la memoria de mi madre... 
"Si buscas resultados distintos, no hagas siempre lo mismo"

Albert Einstein 
ÍNDICE

LISTA DE FIGURAS

vi

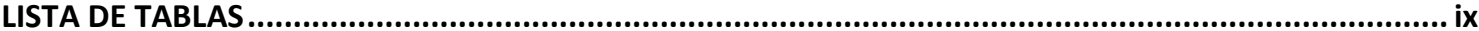

RESUMEN

xiii

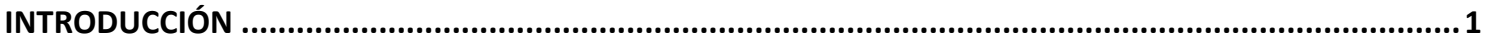

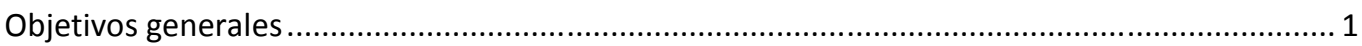

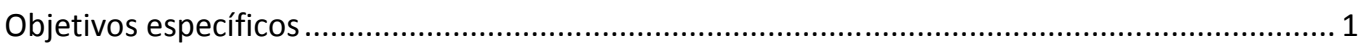

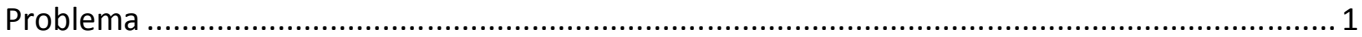

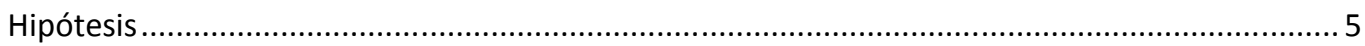

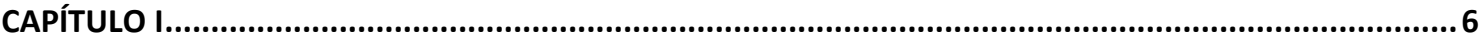

1. ADAPTACIONES FISIOLÓGICAS CARDIOVASCULARES POR EL EJERCICIO .......................................... 6

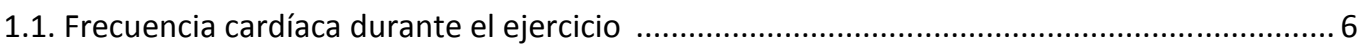

1.2. Volumen sistólico durante el ejercicio ................................................................. 7

1.3. Determinación del gasto cardíaco y del VO2max ........................................................ 8

2. ASPECTOS EPIDEMIOLÓGICOS DE LA ACTIVIDAD FÍSICA Y DE LAS ENFERMEDADES

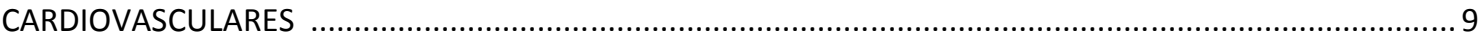

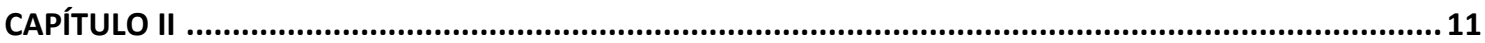

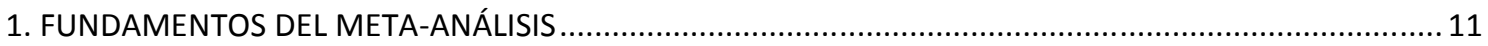

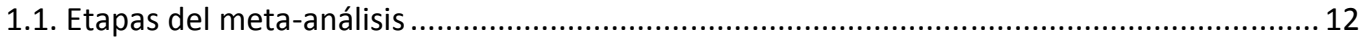

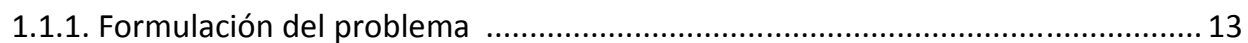

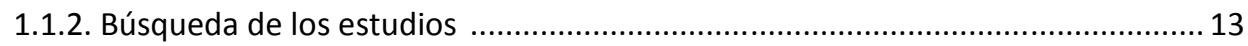

1.1.3. Codificación de los estudios …................................................................. 15

1.1.4. Cálculo del tamaño del efecto ..................................................................... 16

1.1.5. Análisis estadístico e interpretación ............................................................... 19 
1.1. Problema y objetivos 20

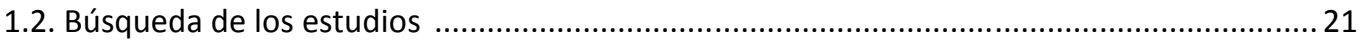

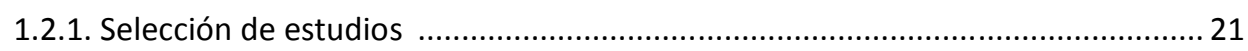

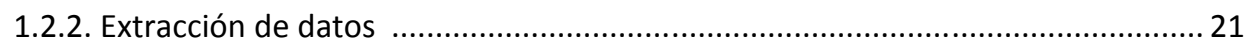

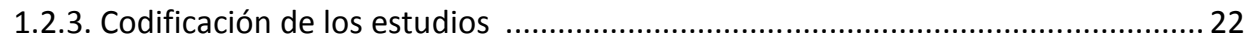

1.2.3.1. Variables moderadoras ........................................................... 23

1.2.4. Cálculo del tamaño del efecto y análisis estadístico .......................................... 24

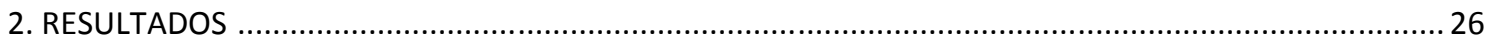

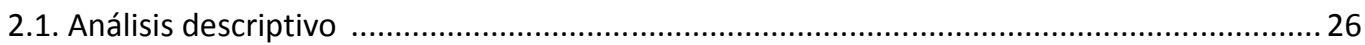

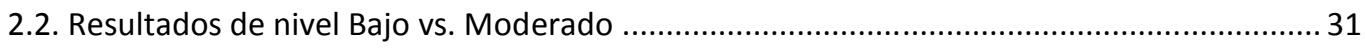

2.2.1. Evaluación de sesgo de publicación ............................................................ 33

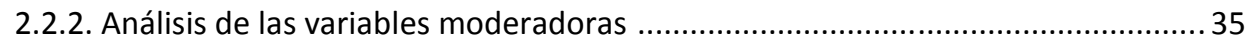

2.2.2.1. Variables sustantivas .............................................................. 35

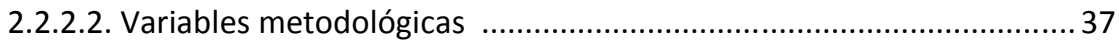

2.2.2.3. Variables extrínsecas ............................................................... 38

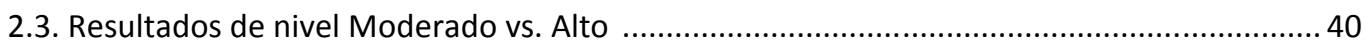

2.3.1. Evaluación de sesgo de publicación ............................................................ 42

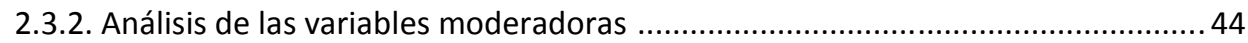

2.3.2.1. Variables sustantivas ................................................................ 44

2.3.2.2. Variables metodológicas .......................................................... 46

2.3.2.3. Variables extrínsecas ............................................................... 47

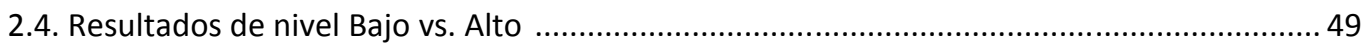

2.4.1. Evaluación de sesgo de publicación ................................................................. 51

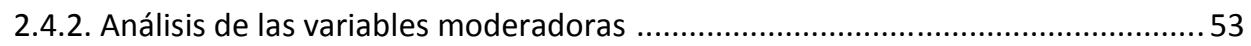

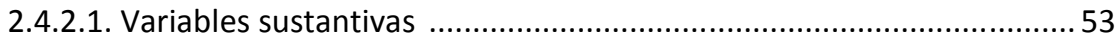


CAPÍTULO IV

1. DISCUSIÓN

2. CONCLUSIÓN 61

BIBLIOGRAFÍA 62

ANEXO I 70

ANEXO II 76 ANEXO III 84 


\section{Lista de Figuras}

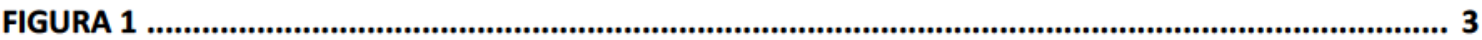

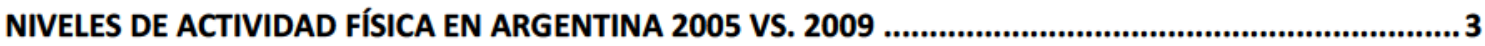

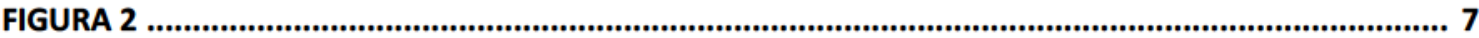

RELACIÓN INTENSIDAD DEL EJERCICIO Y FRECUENCIA CARDÍACA .....................................................

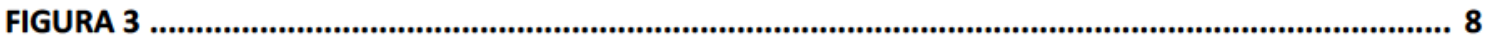

RESPUESTA DEL VOLUMEN SISTÓLICO AL INCREMENTO DE LA INTENSIDAD DEL EJERCICIO ..................... 8

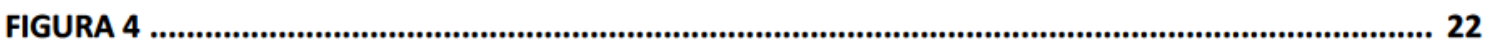

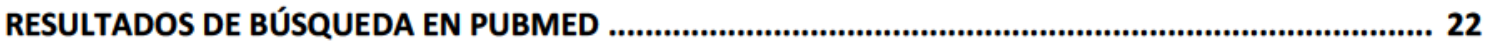

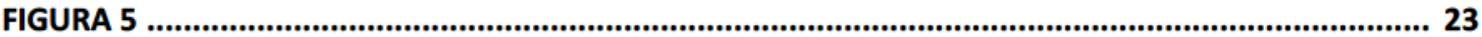

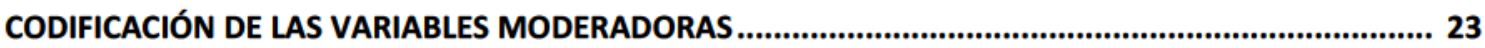

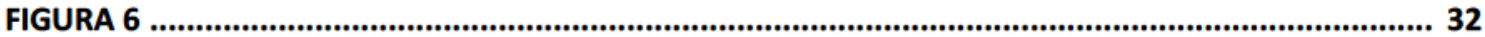

FOREST PLOT DE RIESGO RELATIVO BAJO VS. MODERADO ............................................................ 32

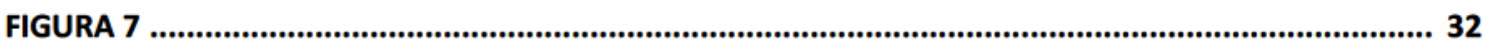

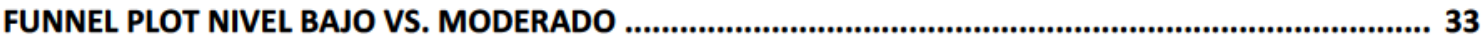

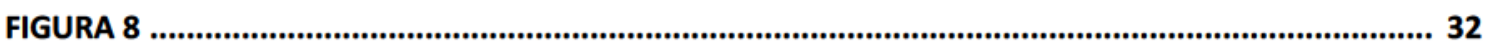

TEST EGGER SOBRE SESGO DE PUBLICACIÓN ...................................................................................... 34

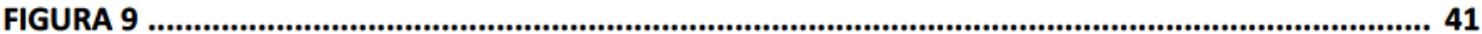

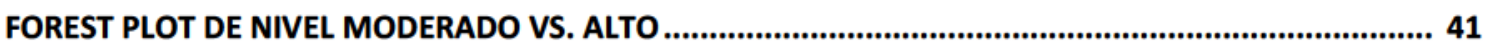

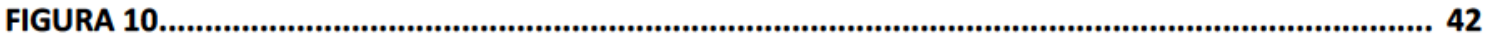

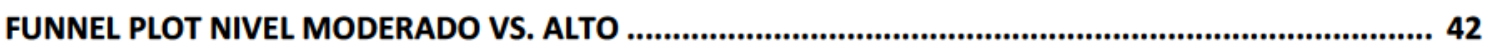

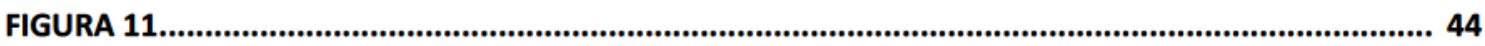

GRÁFICO DE EGGER PARA SESGO DE PUBLICACIÓN ....................................................................... 44 
FIGURA 12

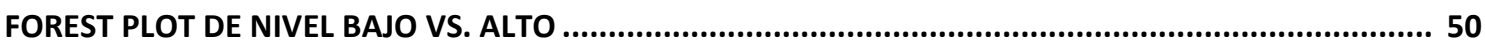

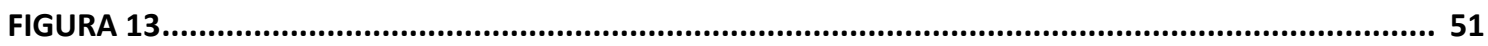

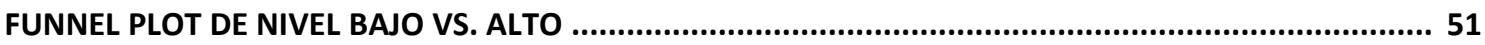

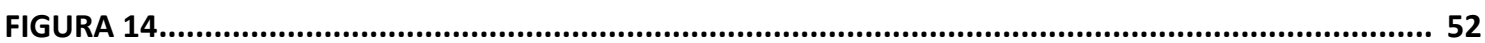

GRÁFICO DE EGGER PARA SESGO DE PUBLICACIÓN ...................................................................... 52

FIGURA 15

FOREST PLOT DE ODDS RATIO BAJO VS. MODERADO ….................................................................. 70

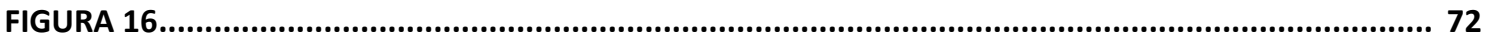

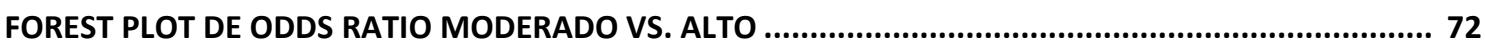

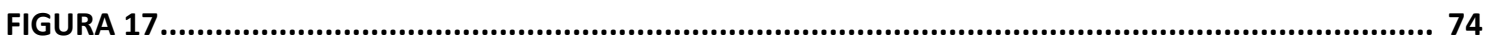

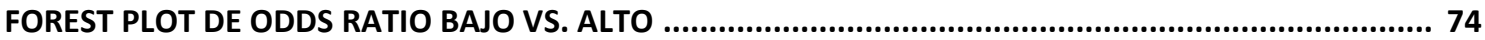

FIGURA 18

META-REGRESIÓN SOBRE LA VARIABLE PORCENTAJE DE VARONES................................................... 75

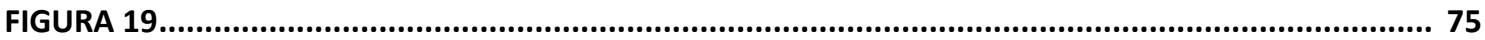

META-REGRESIÓN SOBRE LA VARIABLE EDAD DE LOS PARTICIPANTES .............................................. 75

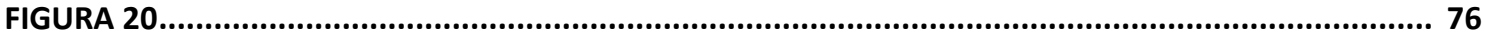

META-REGRESIÓN DE LA VARIABLE TAMAÑO MUESTRAL .............................................................. 76

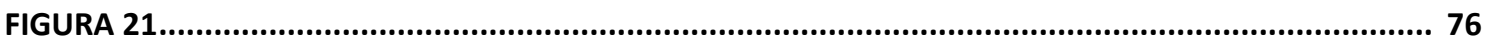

META-REGRESIÓN DE LA VARIABLE AÑOS DE SEGUIMIENTO_......................................................... 76

FIGURA 22

META-REGRESIÓN DE LA VARIABLE AÑO DE PUBLICACIÓN............................................................... 77

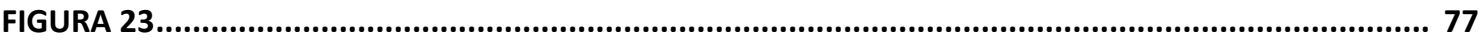


FIGURA 24

META-REGRESIÓN DE LA VARIABLE EDAD MEDIA

FIGURA 25 78

META-REGRESIÓN DE LA VARIABLE TAMAÑO MUESTRAL 78

FIGURA 26 79

META-REGRESIÓN DE LA VARIABLE AÑOS DE SEGUIMIENTO. 79

FIGURA 27 79

META-REGRESIÓN DE LA VARIABLE AÑO DE PUBLICACIÓN. 79

FIGURA 28 80

META-REGRESIÓN DE LA VARIABLE PORCENTAJE DE VARONES 80

FIGURA 29 80

META-REGRESIÓN DE LA VARIABLE EDAD MEDIA 80

FIGURA 30 81

META-REGRESIÓN SOBRE LA VARIABLE TAMAÑO MUESTRAL 81

FIGURA 31 81

META-REGRESIÓN DE LA VARIABLE AÑOS DE SEGUIMIENTO. 81 FIGURA 32 82

META-REGRESIÓN DE LA VARIABLE AÑO DE PUBLICACIÓN. 82 


\section{Lista de Tablas}

TABLA 1

TABLA 2

NIVELES DE EVIDENCIA

TABLA 3

DIAGRAMA DE FLUJO DE LA INFORMACIÓN

TABLA 4

TABLA DE CONTINGENCIA.

TABLA 5

DESCRIPCIÓN DE LA EDAD MEDIA DE LOS PARTICIPANTES POR ESTUDIO

TABLA 6 26

FRECUENCIA DE LA EDAD DE LOS PARTICIPANTES POR ESTUDIO 26

TABLA 7 27

DESCRIPCIÓN DEL TAMAÑO MUESTRAL 27

TABLA 8

TABLA 9

DESCRIPCIÓN DE LOS AÑOS DE PUBLICACIÓN DE LOS ESTUDIOS

FRECUENCIA DE LOS AÑOS DE PUBLICACIÓN DE LOS ESTUDIOS

TABLA 11 28 
TABLA 13

DESCRIPCIÓN DE LA REGIÓN DE ESTUDIO ......................................................................... 29

TABLA 14

TABLA 15

TABLA 17 
META-REGRESIÓN DE LA VARIABLE PORCENTAJE DE VARONES ........................................................ 53

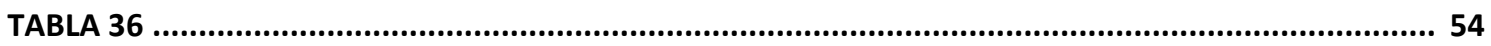

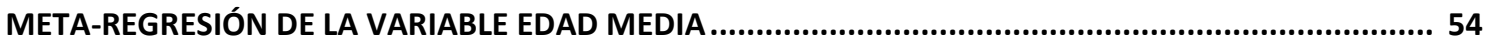

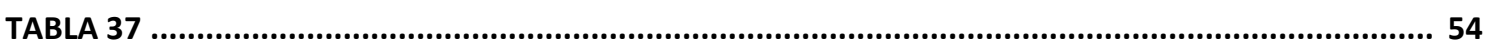

ANÁLISIS DE VARIANZA SOBRE LA VARIABLE PATOLOGÍA CARDÍACA .............................................. 54

TABLA 38

META-REGRESIÓN DE LA VARIABLE TAMAÑO MUESTRAL ............................................................. 55

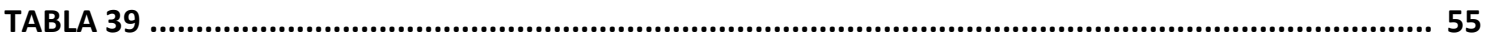

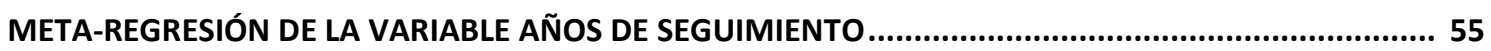

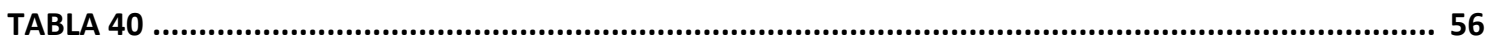

ANÁLISIS DE VARIANZA DE LA VARIABLE REGIÓN DE ESTUDIO ....................................................... 56

TABLA 41

META-REGRESIÓN DE LA VARIABLE AÑO DE PUBLICACIÓN............................................................... 56

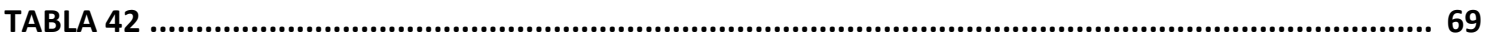

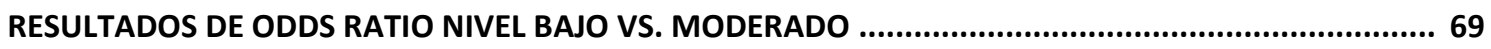

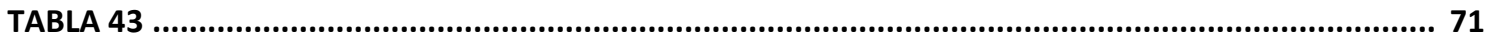

RESULTADOS DE ODDS RATIO NIVEL MODERADO VS. ALTO .......................................................... 71

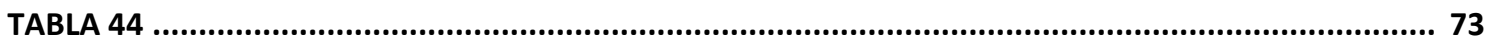

RESULTADO DE ODDS RATIO NIVEL BAJO VS. ALTO ........................................................................ 73

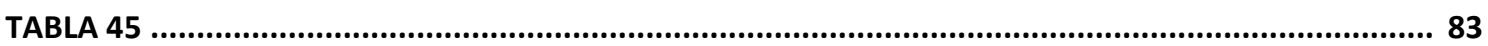

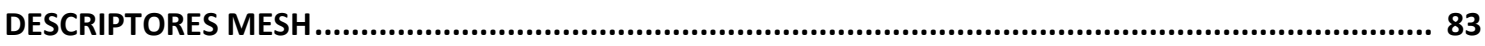




\section{RESUMEN}

\section{Objetivos:}

a. Determinar la eficacia de la actividad física en la reducción del riesgo de padecer enfermedades cardiovasculares.

b. Analizar los cambios relativos en los niveles de riesgo cardiovascular de acuerdo a distintas intensidades de actividad física (Bajo vs. Moderado; Bajo vs. Alto; Moderado vs. Alto).

\section{Metodología:}

La investigación es de tipo cuantitativa, por lo cual, se realizó una revisión sistemática mediante el uso de una técnica estadística denominada meta-análisis. Los meta-análisis se dividieron en tres niveles de actividad física con el objetivo de comparar en cada uno el riesgo relativo, en relación con las enfermedades cardiovasculares (Bajo vs. Moderado; Bajo vs. Alto; Moderado vs. Alto). En primer lugar, se formuló un problema de investigación, con el cual se determinó la ecuación de búsqueda a utilizar y los criterios de inclusión y exclusión de los estudios que fueron identificados. En segundo lugar, se procedió con la búsqueda de estudios aplicando la ecuación de búsqueda en la base de datos PUBMED. Seguido a esto, se codificaron los estudios para proceder a realizar el análisis estadístico. Luego se calculó el tamaño del efecto medio de los estudios incluidos en el meta-análisis y se aplicaron una serie de test a fin de descartar algún posible sesgo de publicación. En los casos que se detectó heterogeneidad, se procedió a realizar un análisis de las variables moderadoras, para las variables continuas se aplicó una meta-regresión, y para las variables categóricas un análisis de varianza. 


\section{Resultados:}

En la primer etapa, se analizaron los niveles de actividad física Bajo vs. Moderado según los METs consumidos en cada estudio siguiendo los criterios de la $\mathrm{OMS}^{1}$, utilizando el modelo de efectos aleatorios. De acuerdo a este análisis, se obtuvo un tamaño del efecto medio de $0.78(0.70-0.86$; $95 \%$ IC), este valor expresa que las personas que realizan actividad física en forma moderada reducen en un $22 \%$ el riesgo de padecer enfermedades cardiovasculares. Luego para evaluar y descartar la posibilidad de un sesgo de publicación, se realizó el test de Egger el cual arrojó un pvalor de 0.67 , en consecuencia, se confirmó la ausencia de sesgo. Por último, debido a la presencia de heterogeneidad, se procedió a realizar un análisis de las variables moderadoras, con el cual se determinó que los años de seguimiento $(\mathrm{p}=0.000 ; 95 \%$ IC) y la región de estudio ( $\mathrm{p}=0.0096 ; 95 \%$ IC) explicaban en parte la heterogeneidad detectada.

En la segunda etapa, se analizaron los niveles de actividad física Moderado vs. Alto utilizando el modelo de efectos aleatorios. En este análisis se observó un tamaño del efecto medio $0.81(0.72-0.91 ; 95 \%$ IC), en consecuencia, este valor expresa que las personas que realizan actividad física en forma vigorosa reducen en un $19 \%$ el riesgo de padecer enfermedades cardiovasculares en relación con las personas que la realizan en forma moderada. Posteriormente, a fin de evaluar y descartar la posibilidad de un sesgo de publicación se realizó el test de Egger con el que se observó un p-valor de 0.394, confirmando así la ausencia de sesgo. Finalmente y debido a la presencia de heterogeneidad, se procedió a realizar un análisis de las variables moderadoras, mediante el cual no se obtuvo significatividad estadística en ninguna de las variables y por ende no se pudo explicar la heterogeneidad detectada.

En la tercer etapa, se analizaron los niveles de actividad física Bajo vs. Alto utilizando el modelo de efectos aleatorios. Se obtuvo un tamaño del efecto medio de 0.61 (0.51$0.74 ; 95 \%$ IC), en función de este resultado, las personas que realizan actividad física en

\footnotetext{
${ }^{1}$ Para la comparación de los niveles de actividad física se utilizó la siguiente clasificación en METs:

1. Bajo: 0 a 3 METs

2. Moderado: 3 a 6 METs

3. Alto: $>6$ METs
} 
forma vigorosa reducen en un 39\% el riesgo de padecer enfermedades cardiovasculares en relación con las personas que realizan actividad física en un nivel bajo. Para descartar un sesgo de publicación, se procedió a realizar el test de Egger obteniéndose un p-valor de 0.304, lo que indica ausencia de sesgo. Al haberse encontrado heterogeneidad en los resultados, se realizó un análisis de las variables moderadoras. De este análisis se desprendió que, las variables años de seguimiento ( $\mathrm{p}=0.009 ; 0.95 \%$ IC) y región de estudio ( $\mathrm{p}=0.0113 ; 95 \%$ IC) explican en parte la heterogeneidad detectada.

\section{Conclusión:}

De acuerdo al tamaño del efecto medio obtenido en el estudio meta-analítico sobre los niveles de actividad física Bajo vs. Moderado, se puede decir que la práctica de actividad física en forma moderada ofrece un efecto protector sobre los individuos que la realizan disminuyendo el riesgo de estos a padecer enfermedades cardiovasculares.

En el caso del análisis de los niveles de actividad física Moderado vs. Alto, de acuerdo al tamaño del efecto medio obtenido, se puede concluir que la práctica de actividad física en niveles altos ofrece un efecto protector sobre los individuos que la realizan disminuyendo el riesgo de estos a padecer enfermedades cardiovasculares en relación con niveles de actividad física moderada.

Por último, evaluados los niveles de actividad física Bajo vs. Alto, se puede concluir que las intensidades altas de actividad física proveen un mayor efecto protector que intensidades moderadas, en relación con las intensidades bajas, y en función de esto disminuyen en un mayor porcentaje el riesgo cardiovascular. 


\section{INTRODUCCIÓN}

\section{Objetivos Generales:}

- Analizar los efectos de la práctica de actividad física sobre la prevención de enfermedades cardiovascular utilizando como herramienta un Meta-análisis.

- Determinar la reducción del riesgo a padecer enfermedades cardiovasculares en sujetos aparentemente sanos.

\section{Objetivos Específicos:}

- Determinar la eficacia de la actividad física en la reducción del riesgo de enfermedades cardiovasculares.

- Analizar los cambios relativos en los niveles de riesgo de padecer enfermedades cardiovasculares de acuerdo a distintas intensidades de actividad física (Baja vs. Moderada; Baja vs. Alta; Moderada vs. Alta).

\section{Problema:}

En la actualidad, el sedentarismo es un problema que va en constante aumento y genera una gran carga en los sistemas de salud pública. La epidemiologia por su parte, ha estudiado la asociación entre diferentes tipos de enfermedades y la inactividad física. Estos estudios han evidenciado que existe una relación muy fuerte entre los estilos de vida sedentarios y diferentes enfermedades (cardiovasculares, metabólicas, musculoesqueléticas) (Lee, Hennekens, Berger, Buring \& Manson, 1999; Rist, Lee, Kase, Gaziano \& Kurth, 2011), de manera tal que la promoción de la actividad física y el deporte toman un lugar importante en el desarrollo de políticas de salud pública.

En el hábitat natural de nuestros antepasados, la actividad física no se consideraba una intervención preventiva, sino una cuestión de la supervivencia. En ese ambiente hostil y con recursos escasos, las vías metabólicas aeróbicas, las funciones cardíacas y las funciones vasculares fueron desafiadas constantemente por episodios intermitentes de alta intensidad de actividad física y fueron adaptándose para satisfacer la demanda 
metabólica del trabajo físico en virtud de esas condiciones (Gielen, Schuler \& Adams, 2010).

Los niveles de actividad física en las sociedades occidentales están por debajo de los niveles recomendados por el Colegio Americano de Medicina del Deporte y por la Asociación Americana del Corazón (Kettel Khan et. al, 2009). En el caso de actividad aeróbica, se recomienda que para promover y mantener la salud, todo los adultos sanos de 18 a 65 años realicen actividad física aeróbica de intensidad moderada durante al menos 30 minutos, en cinco días a la semana o de actividad aeróbica de intensidad vigorosa durante un mínimo de 20 minutos, en tres días a la semana. También se recomienda el entrenamiento de fuerza muscular para promover y mantener una buena salud e independencia física, en consecuencia, explican que los adultos se beneficiarán de la realización de actividades que mantengan o aumenten su resistencia y fuerza muscular durante un mínimo de dos días a la semana. De esta manera, se recomienda la realización de 8 a 10 ejercicios en dos o más días no consecutivos por semana incluyendo los principales grupos musculares, y con una resistencia que permita realizar de 8 a 12 repeticiones en cada ejercicio (Blair et. al, 2007).

A nivel global, la inactividad física es responsable de 3,2 millones de muertes anualmente y aumenta el riesgo de cardiopatía isquémica y enfermedad cerebrovascular, cáncer de mama, cáncer colorectal y diabetes. Se estima a nivel mundial una prevalencia de sedentarismo del $17 \%$, aunque si consideramos también a la actividad física insuficiente, esta cifra asciende al 41\% (ENFR, 2009). 
Figura 1. Niveles de Inactividad Física en Argentina 2005 vs. 2009

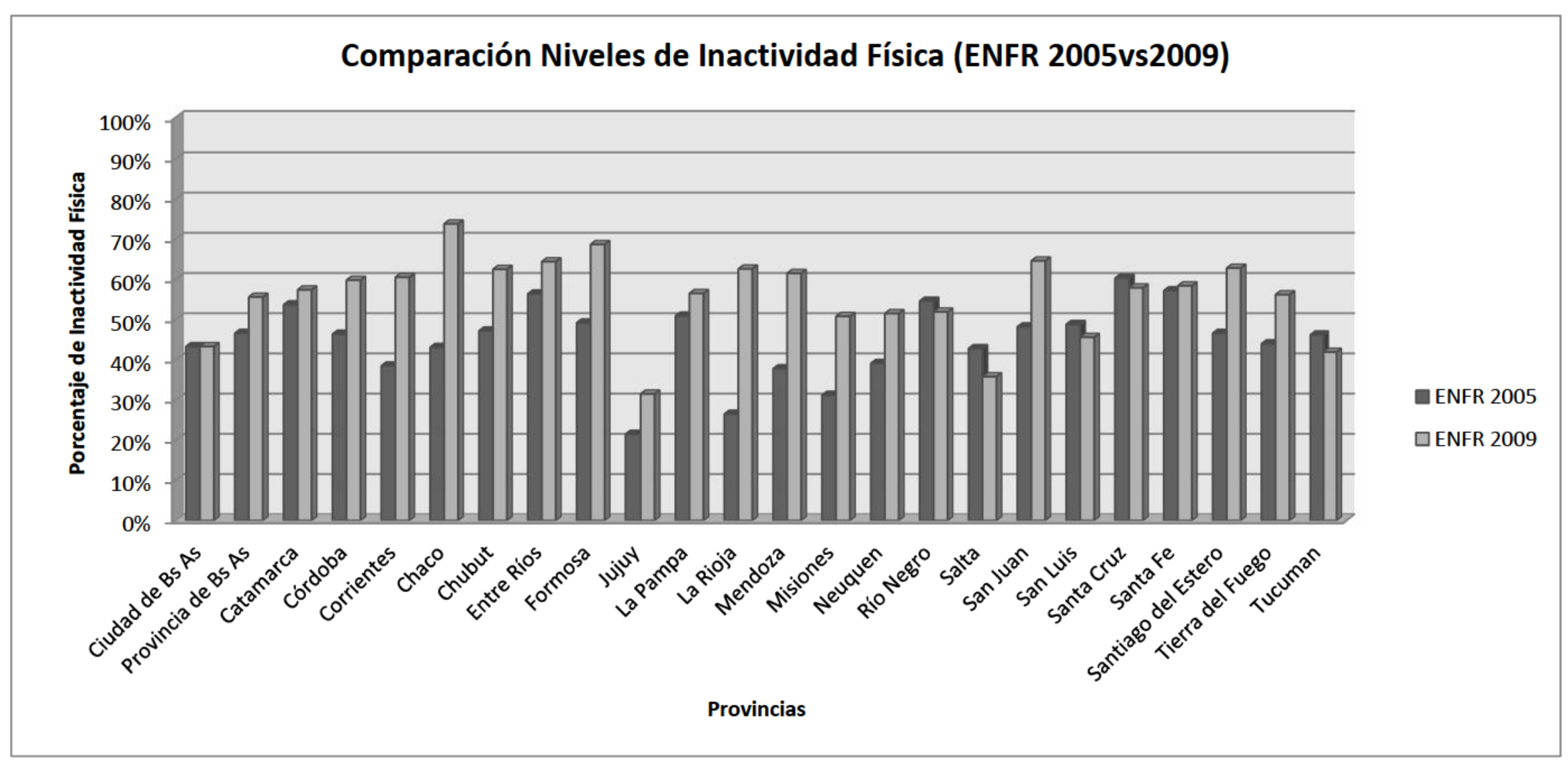

Fuente: Elaboración propia en base a ENFR 2005, 2009. 
En el caso de Argentina, según la Encuesta Nacional de Factores de Riesgo (2009), se observó un aumento en la prevalencia de actividad física baja a nivel nacional del $46.2 \%(2005)$ al $54.9 \%$ (2009).

Un estilo de vida sedentario es considerado un importante factor de riesgo modificable para las enfermedades cardiovasculares en la población general. Los investigadores coinciden en que la actividad física proporciona beneficios cardiovasculares. Una revisión de estudios prospectivos publicados entre 1990 y 2000 llegó a la conclusión de que la reducción en el riesgo de enfermedad coronaria asociado con un estilo de vida físicamente activa, en comparación con un estilo de vida sedentaria, es del $35 \%$ al 55 \%. En consecuencia, la actividad física puede retrasar el inicio y la progresión de la enfermedad a través de efectos favorables sobre el peso corporal, la presión arterial, la sensibilidad a la insulina, el control glucémico y la función endotelial (Skerrett \& Manson, 2002).

Estudios epidemiológicos de cohortes, muestran consistentemente una notable reducción en la incidencia de eventos cardiovasculares entre los individuos físicamente activos en comparación con sus pares inactivos. Estas investigaciones recientes proporcionan apoyo empírico para la prescripción de 30 minutos al día de actividad física de intensidad moderada en poblaciones sedentarias. En estos estudios, los datos de la exposición proceden de cuestionarios de auto-reporte de actividad física. El total de participantes fue de 73.743 mujeres posmenopáusicas de 50 a 79 años que caminaron a paso ligero durante al menos 2,5 horas a la semana. La realización de esta actividad se asoció con una reducción del $30 \%$ en los eventos cardiovasculares en un total de 3,2 años de seguimiento (Skerrett \& Manson, 2002).

Debido a la elevada prevalencia de inactividad física $\mathrm{y}$ de enfermedades cardiovasculares en la población en general, resulta pertinente investigar la eficacia de la actividad física como medida preventiva para reducir la probabilidad de padecer enfermedades cardiovasculares. Por lo tanto, el objetivo principal de este trabajo de tesis es contribuir a la producción de evidencia sobre los beneficios de la actividad física en la salud, específicamente sobre la prevención de enfermedades cardiovasculares. 


\section{Hipótesis:}

La inactividad física es uno de los principales factores de riesgos asociados a enfermedades cardiovasculares. El aumento de la aptitud física disminuye los factores de riesgo cardiovascular. Sin embargo, niveles vigorosos de actividad física (medidos en términos de METs) producen una disminución más pronunciada del riesgo relativo asociado a enfermedades cardiovasculares que niveles bajos e incluso moderados. 


\section{CAPÍTULO I}

\section{Adaptaciones fisiológicas cardiovasculares por el ejercicio:}

Según Vaquero (2006), cuando realizamos ejercicio, el sistema cardiovascular sufre una serie de adaptaciones que se reflejan en un aumento del gasto cardíaco, producto de esto, la cantidad de sangre oxigenada que expulsa el ventrículo izquierdo aumenta en referencia a la cantidad eyectada del ventrículo izquierdo en reposo. El aumento del gasto cardíaco no se produce en forma lineal, sino que depende de ciertos factores que influyen sobre el mismo, en función de esto se puede mencionar que la masa muscular involucrada, la intensidad del ejercicio y la capacidad que posee el corazón para aumentar su volumen sistólico son factores fundamentales para determinar el aumento del gasto cardíaco ante un determinado ejercicio. Siendo el gasto cardíaco, una variable explicativa del consumo máximo de oxígeno, se debe mencionar que para la determinación del mismo, se debe sumar la diferencia arteriovenosa de oxígeno. Esta variable tiene menor influencia en el VO2max, ya que depende de factores periféricos, en cambio el gasto cardíaco, depende en su mayor parte del corazón, teniendo como componentes más importantes el volumen sistólico y la frecuencia cardíaca (López Chicharro \& Fernández Vaquero, 2006).

\subsection{Frecuencia cardíaca durante el ejercicio}

Como se observa en la figura 2, el aumento de la frecuencia cardíaca durante el ejercicio se produce de manera lineal en relación a la intensidad con la que se realiza, hasta llegar a la frecuencia cardíaca máxima. Al realizar un esfuerzo sub-máximo, la frecuencia cardíaca aumenta rápidamente hasta que se estabiliza en un punto denominado "estado estable de la frecuencia cardíaca", donde el corazón ha alcanzado el ritmo óptimo para satisfacer los requerimientos del sistema circulatorio a una determinada intensidad del ejercicio. Cuando se alcanza la estabilización de la frecuencia cardíaca, y se incrementa la intensidad del ejercicio, se produce un aumento de la frecuencia cardíaca tardando aproximadamente entre 1 a 2 minutos en estabilizarse nuevamente, sin embargo cabe destacar que a mayor intensidad del ejercicio, llegar al punto donde se estabiliza la frecuencia cardíaca demanda mayor tiempo (Wilmore \& Costill, 2004). 
Figura 2. Relación intensidad de ejercicio y frecuencia cardíaca

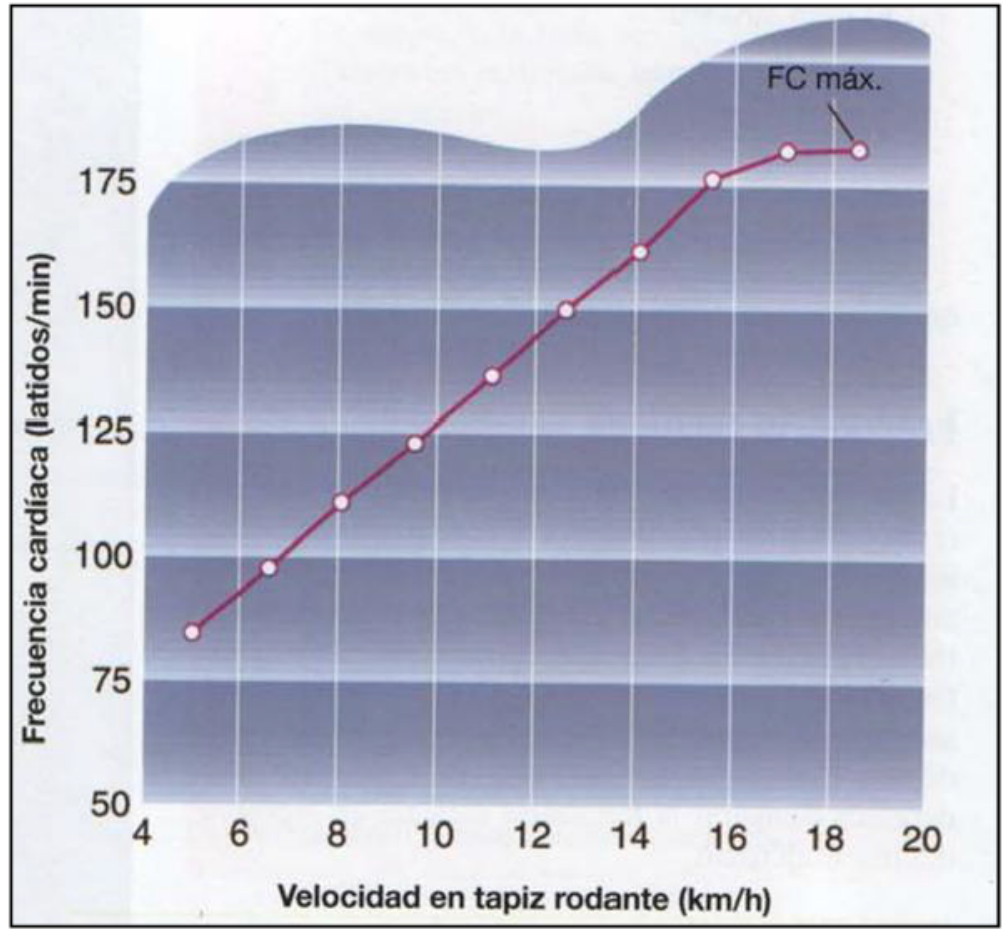

Fuente: Wilmore \& Costill 2004.

\subsection{Volumen sistólico durante el ejercicio}

Otro factor que influye sobre el gasto cardíaco es el volumen sistólico. Cuando se realiza ejercicio, el volumen sistólico se incrementa de manera no lineal en función de la intensidad a la que se realiza el esfuerzo físico. Así mismo, la respuesta del volumen sistólico frente al ejercicio puede depender de muchos factores, incluyendo la edad, condición física, nivel de entrenamiento, el sexo y los factores genéticos. En sujetos sedentarios, el volumen sistólico comienza a incrementarse de acuerdo con la intensidad del ejercicio hasta que llega a un punto de estabilización que se encuentra entre el $50 \%$ y el $60 \%$ del VO2max. (Vella \& Robergs, 2005) 
Figura 3. Respuesta del Volumen Sistólico al incremento de la intensidad del ejercicio

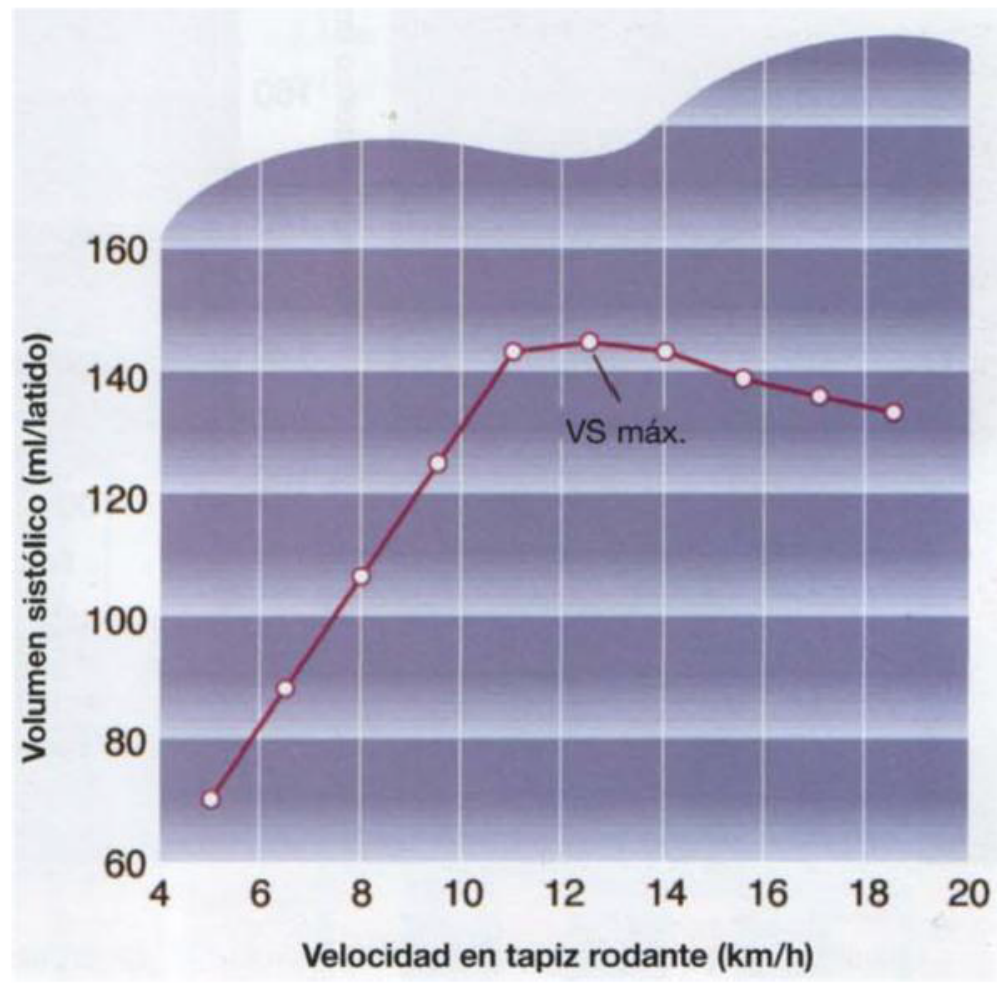

Fuente: López Chicharro 2006.

En un estudio realizado sobre sujetos (hombres y mujeres) sanos y sedentarios, se planteó un entrenamiento aeróbico de 12 semanas y se documentó que, antes del entrenamiento, el volumen sistólico de los sujetos cayó un 9\% cuando la intensidad del ejercicio se incrementó desde el 50\% hasta el 100\% del VO2max. Luego del período de entrenamiento los mismos sujetos mostraron una disminución del volumen sistólico del 2\%. En función de esto, se concluyó que el entrenamiento disminuyó la caída del volumen sistólico frente a intensidades elevadas de ejercicio aeróbico (Spina et. al, 1992).

\subsection{Determinación del gasto cardíaco y del VO2max:}

Como se explicó anteriormente, la producción cardíaca (Gasto cardíaco) está determinada en función del producto entre la frecuencia cardíaca y el volumen sistólico, de manera tal que:

$$
Q=F C x V S
$$


donde $Q$ es la producción cardíaca, $F C$ es la frecuencia cardíaca y $V S=V D F-V S F^{2}$, es la diferencia entre volumen diastólico final y el volumen sistólico final.

Obteniendo la producción cardíaca, se puede calcular el volumen máximo de oxígeno según la ecuación de Fick:

$$
\mathrm{VO}_{2}=\mathrm{GC} \times \mathrm{D}(a-v) \mathrm{O}_{2}
$$

Donde, $G C$ es el gasto cardíaco y $D(a-v) O_{2}$ es la diferencia arteriovenosa de oxígeno $^{3}$. Es importante destacar que existe variada evidencia epidemiológica que establece una relación de asociación positiva entre el aumento del nivel de VO2max y la disminución en los niveles de riesgo de cardiopatías coronarias (Jette, Sidney, Quenneville \& Landry, 1992; McMurray, Ainsworth, Harrell, Griggs \& Williams, 1998).

\section{Aspectos epidemiológicos de la actividad física y las enfermedades cardiovasculares:}

El ejercicio físico regular tiene efectos beneficiosos en la salud y el bienestar de las personas. Un número cada vez mayor de estudios epidemiológicos confirman la relación inversa entre la actividad física y la incidencia de enfermedades coronarias (Morris, Everitt \& Pollard, 1980; Blair, Kohl \& Paffenbarger, 1989; Siscovick, LaPorte \& Newman, 1985; Paffenbarger, Hyde \& Wing, 1986; Paffenbarger, Hyde \& Wing, 1990; Leon et. al, 1987; Sobolski et. al, 1987; Slattery \& Jacobs, 1988; Ekelund et. al, 1988; Powell et. al, 1987). Además, el infarto de miocardio no sólo ocurre con más frecuencia entre las personas sedentarias que entre las personas activas, sino que también es más fatal y a menudo se produce a una edad mucho menor (Council of Europe, 1970; Siscovick et. al, 1982). Hay fuerte consistencia estadística apoyada por resultados experimentales de laboratorio, que sostienen el argumento de que la actividad física

\footnotetext{
${ }^{2}$ Se denomina volumen diastólico final a la sangre que se encuentra en el ventrículo izquierdo al final de la diástole y justo antes de que se produzca la contracción. Luego de la contracción, se ha completado la fase de eyección, y al volumen de sangre restante en el ventrículo se lo denomina como volumen sistólico final (Wilmore \& Costill, 2004).

${ }^{3}$ La diferencia arteriovenosa de oxígeno representa la medida en que es extraído o eliminado el oxígeno de la sangre a medida que pasa por el cuerpo (Wilmore \& Costill, 2004).
} 
regular y vigorosa juega un papel fundamental en la prevención del infarto de miocardio (Siscovick et. al, 1990).

Los efectos del entrenamiento físico incluyen la reducción de los niveles de colesterol total y del colesterol de baja densidad, el aumento de los niveles de colesterol de lipoproteína de alta densidad, el aumento de la densidad de receptores de insulina, el aumento de la depuración de glucosa, y una disminución de la presión sanguínea (Jetté et. al, 1992).

En cuanto a la intensidad del ejercicio, varias líneas de evidencia sugieren mayores adaptaciones aeróbicas y cardiovasculares en sujetos sanos después del ejercicio de alta intensidad que con niveles bajos y moderados (Lee, Sesso, Oguma \& Paffenbarger, 2003). Hay evidencia que apoya la idea de que los aspectos centrales de la adaptación del miocardio por el ejercicio dependen de la intensidad del programa de entrenamiento (Kemi et. al, 2005). El ejercicio de intensidad vigorosa ha demostrado mayor eficacia para el aumento de la aptitud aeróbica que el ejercicio de intensidad moderada, lo que sugiere que el primero puede conferir mayores beneficios cardioprotectores (Swain \& Franklin, 2006). 


\section{CAPÍTULO II}

\section{FUNDAMENTOS DEL META-ANÁLISIS}

Un meta-análisis constituye un tipo de revisión sistemática. Proporciona un conjunto de técnicas estadísticas que combina resultados de todos los estudios que constituyan parte de la revisión con el objetivo de contestar una pregunta científica relacionada con el problema de investigación (Naylor, 1997).

Tabla 1. Revisiones sistemáticas y Meta-análisis

\begin{tabular}{|l|l|}
\hline REVISIÓN & $\begin{array}{l}\text { Término general para todo intento que sintetice los resultados y las } \\
\text { conclusiones de dos o más publicaciones relacionadas con un tema } \\
\text { determinado. }\end{array}$ \\
\hline SISTEMÁTICA & $\begin{array}{l}\text { Cuando una revisión se esfuerza en la identificación exhaustiva, a } \\
\text { través de toda la literatura de un tema dado, valorando su calidad y } \\
\text { sintetizando sus resultados. }\end{array}$ \\
\hline META- & $\begin{array}{l}\text { Revisión sistemática que incorpora una estrategia estadística } \\
\text { específica para reunir los resultados de varios estudios en una sola } \\
\text { estimación. }\end{array}$ \\
\hline
\end{tabular}

Fuente: Adaptado de Sackett 1991.

En un principio, entre las décadas del ' $30 \mathrm{y}$ del ' 50 , este tipo de análisis estadístico empezó a utilizarse en disciplinas de las ciencias sociales. En 1976, el término metaanálisis fue utilizado por primera vez por Glass para indicar el análisis estadístico integrado de resultados de muchos estudios individuales (Zhou, 2002). Más adelante se utilizó en las ciencias de la salud para evaluar la eficacia de tratamientos combinando los resultados de ensayos clínicos, generalizando posteriormente su aplicación a estudios observacionales (Letón Molina, 2001). Según Rodríguez Delgado (2002), el meta-análisis es la sistemática identificación, valoración, síntesis, y la combinación estadística, de todos los estudios sobre un mismo tema, siguiendo un método explícito y determinado.

Sin embargo, no todos los tipos de meta-análisis presentan el mismo grado de evidencia científica. Los ensayos clínicos aleatorizados ofrecen una evidencia de mayor calidad 
que los estudios de cohortes, proporcionando estos a su vez mayor evidencia que los estudios de casos y controles (Schulz, 1998). Los niveles de evidencia científica de revisiones sistemáticas y meta-análisis se pueden clasificar de la siguiente manera:

\section{Tabla 2. Niveles de Evidencia}

\begin{tabular}{|l|l|}
\hline $1++$ & $\begin{array}{l}\text { Meta-análisis, revisiones sistemáticas de ensayos clínicos de alta calidad con muy } \\
\text { poco riesgo de sesgo. }\end{array}$ \\
\hline $1+$ & $\begin{array}{l}\text { Meta-análisis, revisiones sistemáticas de ensayos clínicos o ensayos clínicos bien } \\
\text { realizados con poco riesgo de sesgo. }\end{array}$ \\
\hline $1-$ & $\begin{array}{l}\text { Meta-análisis, revisiones sistemáticas de ensayos clínicos o ensayos clínicos con } \\
\text { alto riesgo de sesgo. }\end{array}$ \\
\hline $2++$ & $\begin{array}{l}\text { Revisiones sistemáticas de estudios de cohortes o de casos y controles o estudios } \\
\text { de pruebas diagnósticas de alta calidad, estudios de cohortes o de casos y } \\
\text { controles de pruebas diagnósticas de alta calidad con riesgo muy bajo de sesgo y } \\
\text { con alta probabilidad de establecer una relación causal. }\end{array}$ \\
\hline $2+$ & $\begin{array}{l}\text { Estudios de cohortes o de casos y controles o estudios de pruebas diagnósticas } \\
\text { bien realizadas con bajo riesgo de sesgo y con una moderada probabilidad de } \\
\text { establecer relación causal. }\end{array}$ \\
\hline $2-$ & Estudios de cohortes o de casos y controles con alto riesgo de sesgo. \\
\hline 3 & Estudios no analíticos, como informes de casos y series de casos. \\
\hline 4 & Opinión de expertos. \\
\hline
\end{tabular}

Fuente: Adaptado de SIGN 50: "A guideline developers hand-book. Publication ${ }^{\circ}$ 50, 2001."

\subsection{Etapas del Meta-análisis}

Las etapas que deben llevarse a cabo para realizar un meta-análisis varían en algunos puntos según el autor que las detalle, en este caso utilizamos las etapas que describe Sánchez Meca (2010), en términos generales son:

1. Formulación del problema.

2. Búsqueda de los estudios.

3. Codificación de los estudios. 
4. Cálculo del tamaño del efecto.

5. Análisis estadístico e interpretación.

\subsubsection{Formulación del problema}

En esta etapa, se debe formular una pregunta clara y precisa acerca del problema a solucionar. Luego de construida la pregunta del problema, se debe proseguir determinando los objetivos a concluir por el meta-análisis, y por último, precisar la hipótesis a verificar. En el ámbito de las ciencias sociales y de la salud, las preguntas de investigación son diversas, pero normalmente la pregunta apunta a verificar la eficacia de algún programa, intervención, o tratamiento (Cooper, 2010).

\subsubsection{Búsqueda de los estudios}

Dentro de esta fase, se deben construir los criterios de inclusión y exclusión de los estudios a seleccionar. Se debe aclarar el idioma en el que estarán escritos los estudios y el rango temporal de la búsqueda. También deben contener información sobre los tipos de estudios que se aceptaran, por ejemplo estudios experimentales (Ensayos clínicos), o estudios observacionales (Estudios de cohortes, casos y controles). Se deben definir las características que debe tener la intervención, el programa o el tratamiento, a fin de ser incluido en el meta-análisis. En el punto siguiente se deben especificar las características de los participantes de los estudios. Y por último, determinar cuáles son los datos estadísticos que deben contener estos estudios para ser incluidos. Luego de construidos los criterios de inclusión y exclusión, se procederá a realizar la búsqueda electrónica en diferentes bases de datos, PubMed, Eric, SportDicus, etc. El diagrama de flujo de la información se construye luego de la búsqueda electrónica, identificación, y evaluación de los estudios respecto de los criterios definidos anteriormente. Este en términos generales debe contener la siguiente información: 
Tabla 3. Diagrama de flujo de la información

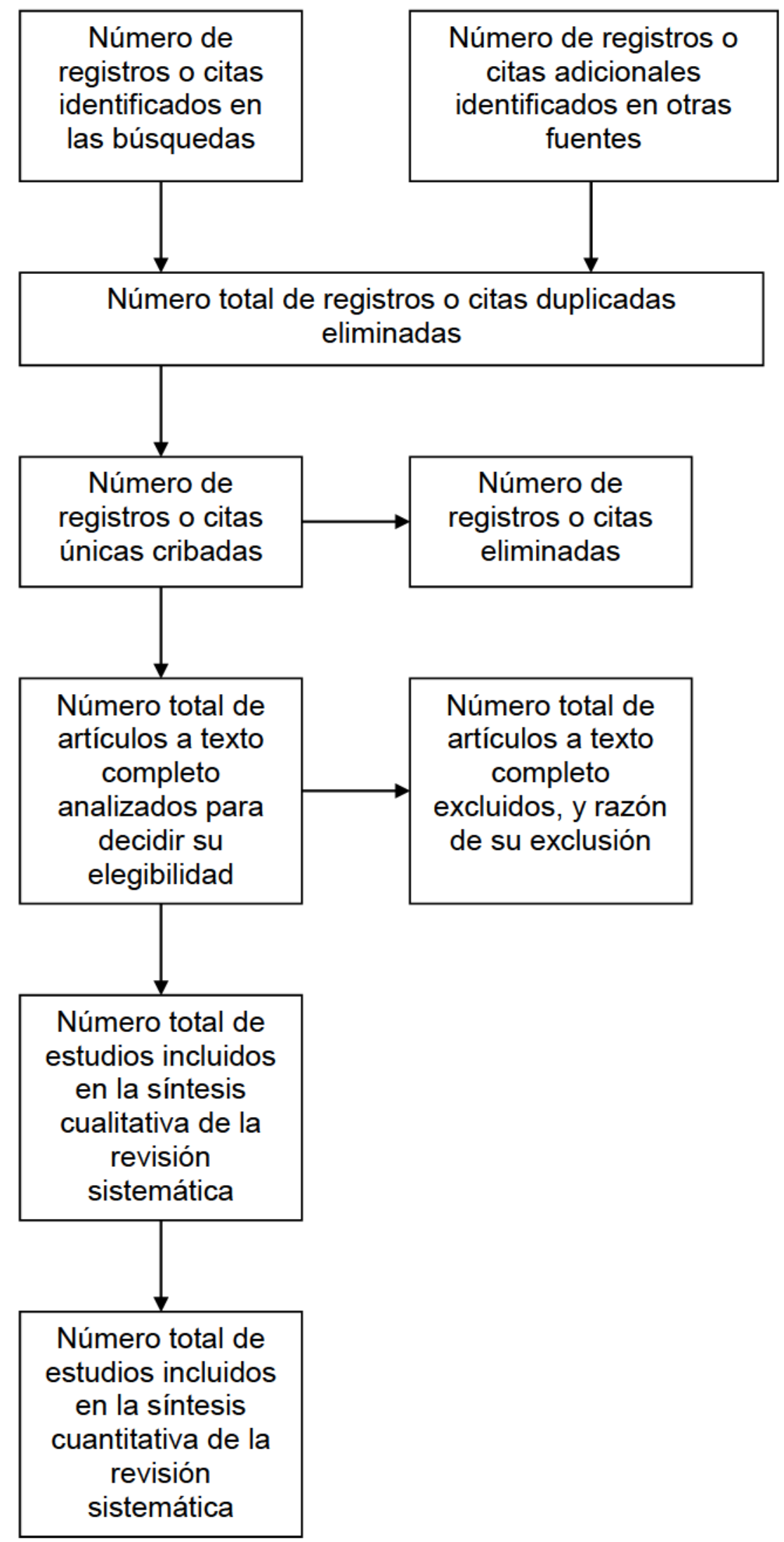

Fuente: Adaptado de la declaración PRISMA 2010 


\subsubsection{Codificación de los estudios}

La codificación de los estudios permite traducir la información en valores adecuados para ser computados y realizar el análisis estadístico, es decir, tiene por objetivo crear variables a partir de los datos en estudio. Para esto, se deben construir algunos interrogantes:

- ¿Qué información existe?

- ¿Qué información es relevante?

- ¿Cómo será analizada? (Schoenbach \& Víctor, 2004)

Luego se procederá a elaborar un manual de codificación donde se explicarán los criterios por medio de los cuales se codificarán las características de los estudios. La razón de estudiar las características consiste en tratar de verificar si están afectando o moderando los resultados del estudio (Lipsey \& Wilson, 2001). A partir del manual de codificación se desarrolla un protocolo de registro de las variables moderadoras. Estas pueden clasificarse según sus características de la siguiente manera:

1. Variables sustantivas.

2. Variables metodológicas.

3. Variables extrínsecas.

Las variables sustantivas son variables moderadoras que están relacionadas con el objeto de estudio del meta-análisis. A su vez, en los meta-análisis que estudian la eficacia de un programa, tratamiento o intervención, estas variables sustantivas se subdividen en características del tratamiento, de los participantes y del contexto. Por su parte, las variables metodológicas se relacionan con el diseño del estudio. Entre estas, se pueden incluir el tipo de diseño, el tamaño muestral, y medidas de pretest y postest. Por último, las variables extrínsecas son las que no necesariamente tendrían que afectar los resultados del estudio, ya que no tienen que ver con el método de investigación, pero en ocasiones, se observa correlación entre estas. Son ejemplo de dichas variables la fuente de publicación y el año del estudio. (Sánchez Meca \& Botella, 2010) 


\subsubsection{Cálculo del tamaño del efecto}

El tamaño del efecto es un índice estadístico que mide el grado en el que difieren los estudios que se están integrando, por lo tanto, se puede definir como cualquier medida estadística que evidencia el grado con el que un evento dado está presente en la muestra (Cohen, 1988). Este estadístico al calcularse debe poder homogeneizar a todos los estudios en la misma unidad de medida de forma tal que los resultados puedan integrarse en una métrica estandarizada.

Se han propuesto varios índices estadísticos para calcular el tamaño del efecto, dependiendo de los objetivos del investigador y del tipo de variable que se esté analizando. Uno de ellos es el índice propuesto por Cohen denominado índice de la familia d, que es utilizado cuando se estima el tamaño del efecto en variables continuas, se calcula de la siguiente manera:

$$
d=c(m) \frac{\overline{y_{t}}-\overline{y_{c}}}{S}
$$

donde $\overline{y_{t}}$ es la media del grupo tratado, $\overline{y_{c}}$ es la media del grupo control, y S es la desviación conjunta de los dos grupos que se calcula:

$$
S=\sqrt{\frac{\left(n_{t}-1\right) S_{t}^{2}+\left(n_{c}-1\right) S_{c}^{2}}{n_{t}+n_{c}-2}}
$$

donde $\mathrm{n}_{\mathrm{t}}$ es el tamaño muestral del grupo tratado, $\mathrm{n}_{\mathrm{c}}$ es el tamaño muestral del grupo control, $\mathrm{S}_{\mathrm{t}}^{2}$ es la desviación estándar al cuadrado del grupo tratado y $\mathrm{S}_{\mathrm{c}}^{2}$ es la desviación estándar al cuadrado del grupo control. Por último, c(m) es un factor de corrección para muestras pequeñas que se calcula:

$$
c(m)=1-\frac{3}{4 N-9}
$$

donde $N=n_{t}+n_{c}$. 
En el caso de que los estudios analizados utilicen medidas de pretest y postest en el grupo control y el grupo tratado, se debe calcular la diferencia de medias de cada grupo, siendo la diferencia de medias del grupo tratado:

$$
\delta_{t}=\frac{\mu_{t, p o s t}-\mu_{t, p r e}}{\sigma}
$$

donde $\mu_{\mathrm{t} \text {,post }}$ es la media de las medidas postest del grupo tratado, $\mu_{\mathrm{t}, \mathrm{pre}}$ es la media de las medidas de pretest del grupo tratado, y $\sigma$ es el desvío estándar pretest del grupo tratado. Y la diferencia de medias del grupo control:

$$
\delta_{c}=\frac{\mu_{c, p o s t}-\mu_{c, p r e}}{\sigma}
$$

donde $\mu_{c, p o s t}$ es la media de las medidas de postest del grupo control, $\mu_{c \text {,pre }}$ es la media de las medidas pretest del grupo control y $\sigma$ es el desvío estándar pretest del grupo control. Para calcular la diferencia de medias conjunta se utiliza el siguiente estadístico (Becker, 1988; Carlson \& Schmidt, 1999; Morris \& DeShon, 2002):

$$
d_{p p c=c_{p}}\left[\frac{\left(M_{\text {post }, t}-M_{\text {pre }, t}\right)-\left(M_{\text {post }, c}-M_{\text {pre }, c}\right)}{S D_{p}}\right]
$$

donde $\mathrm{M}_{\text {post, } \mathrm{t}}$ es la media del grupo tratado postest, $\mathrm{M}_{\text {pre,t }}$ es la media del grupo tratado pretest, $\mathrm{M}_{\text {post,c }}$ es la media del grupo control postest, $\mathrm{M}_{\mathrm{pre}, \mathrm{c}}$ es la media del grupo control pretest, y SD p (Carlson \& Schmidt, 1999; Morris, 2003) es el desvío estándar conjunto de los dos grupos que se calcula:

$$
S D_{p}=\sqrt{\frac{\left(n_{t}-1\right) S D_{p r e, t}^{2}+\left(n_{c}-1\right) S D_{p r e, c}^{2}}{n_{t}+n_{c}-2}}
$$

siendo $\mathrm{n}_{\mathrm{t}}$ el tamaño muestral del grupo tratado, $\mathrm{n}_{\mathrm{c}}$ el tamaño muestral del grupo control, $\mathrm{SD}_{\text {pre,t }}^{2}$ el desvío estándar pretest del grupo tratado elevado al cuadrado y $\mathrm{SD}_{\mathrm{pre}, \mathrm{c}}^{2}$ el desvío estándar pretest del grupo control elevado al cuadrado. 
Por último, se aplica el factor de corrección para muestras pequeñas $c_{p}$ que se calcula (Hedge, 1981):

$$
c_{p}=1-\frac{3}{4\left(n_{t}+n_{c}-2\right)-1}
$$

En el caso que se utilice una variable dicotómica se puede aplicar para calcular el tamaño del efecto el estadístico de riesgo relativo en una tabla de contingencia $2 \times 2$ :

Tabla 4. Tabla de contingencia

\begin{tabular}{|c|c|c|c|}
\hline & EXPERIMENTAL & CONTROL & TOTAL \\
\hline EXPUESTOS & Maris & Feo & $\frac{2}{a}+b$ \\
\hline NO EXPUESTOS & & $\frac{b}{d}$ & $\frac{a}{a+b}$ \\
\hline TOTAL & $\overline{n e}=\bar{a}=0$ & $\bar{n}^{c}=b+d$ & $\begin{array}{c}a+b \\
c+a \\
\sim \sim-a+b+c+d\end{array}$ \\
\hline
\end{tabular}

$$
R R=\frac{\frac{a}{n_{e}}}{\frac{b}{n_{c}}}
$$

donde los valores de $R R=[0 ; \infty)$. Si $R R=1$, se interpreta como ausencia de efecto, si $R R<1$, indica un efecto protector del factor estudiado, y si $R R>1$ indica que los individuos expuestos tienen una mayor probabilidad de enfermedad (Letón Molina, 2001).

Otra medida para calcular el tamaño del efecto es Odds Ratio, Letón Molina la define como:

“... el cociente entre una proporción y su complemento" (Letón Molina, 2001:13)

$$
O R=\frac{\frac{p_{1}}{\left(1-p_{1}\right)}}{\frac{p_{2}}{\left(1-p_{2}\right)}}=\frac{a \cdot d}{b \cdot c}
$$


Si bien esta medida de efecto es similar al riesgo relativo, no es comparable. Si $O R<1$ indica un factor de protección, si $O R>1$ los individuos expuestos tienen mayor probabilidad de enfermedad.

\subsubsection{Análisis estadístico e interpretación}

Luego de codificar las variables moderadoras y haber seleccionado el índice estadístico para realizar el cálculo del tamaño del efecto, se debe crear una base de datos en la que se incluyan los nombres de los estudios, año de publicación, variables moderadoras y tamaño del efecto. Con esta base de datos se realiza el análisis estadístico donde se debe calcular el índice del tamaño del efecto medio de los estudios incluidos, para esto se puede utilizar el modelo de efectos fijos o el modelo de efectos aleatorios, más adelante se hará una descripción de los mismos. En segundo lugar, se debe calcular la heterogeneidad de los tamaños del efecto de cada estudio, y en caso de ser significativa, se deben analizar las variables moderadoras que pueden influir en esta heterogeneidad. (Marín Martínez, Sánchez Meca \& López, 2009) 


\section{CAPÍTULO III}

\section{INTRODUCCIÓN AL META-ANÁLISIS DE ACTIVIDAD FÍSICA Y ENFERMEDADES CARDIOVASCULARES}

\subsection{Problema y objetivos}

Las enfermedades cardiovasculares, incluyendo la enfermedad coronaria y el accidente cerebrovascular, son los mayores contribuyentes de carga de enfermedad en todo el mundo. En la actualidad son la causa más importante de muertes, de producción de discapacidad y disminución de la calidad de vida de las personas sobrevivientes (Yusuf, Reddy, Ounpuu \& Anand, 2001). Existen diferentes factores de riesgo que se asocian a enfermedades cardiovasculares. Se ha observado en amplia literatura científica que el sedentarismo es un factor de riesgo predominante y que la práctica de actividad física puede reducir el riesgo de morbilidad y mortalidad cardiovascular (Sofi, Capalbo, Cesari, Abbate \& Gensini, 2008). Esta evidencia incluye efectos protectores consistentes de la actividad física contra enfermedades coronarias, y menos consistentes contra los accidentes cerebrovasculares (Wendel, et. al, 2004). Por lo dicho anteriormente, se intentará responder a las siguientes preguntas:

¿Es eficaz la práctica de actividad física para reducir el riesgo de padecer enfermedades cardiovasculares?

¿Cuál es el nivel de reducción del riesgo relativo que proporciona la práctica de actividad física en enfermedades cardiovasculares?

¿Existen diferencias en la reducción del riesgo relativo entre los niveles de actividad física Bajo, Moderado y Alto?

, Para responder a estas preguntas se plantean los siguientes objetivos dentro de este meta-análisis:

- Verificar la relación entre la práctica de actividad física y la disminución del riesgo relativo a padecer enfermedades cardiovasculares en estudios de cohortes.

- Diferenciar la modificación del riesgo relativo entre diferentes niveles de actividad física (Bajo, Moderado, Alto). 


\subsection{Búsqueda de los estudios}

\subsubsection{Selección de estudios}

Para seleccionar los estudios a incluir en el meta-análisis se establecieron los siguientes criterios de inclusión:

1. Los estudios científicos deben estar escritos en idioma inglés y español.

2. El diseño de los estudios debe ser de tipo observacional y estar constituido en forma de estudios prospectivos, casos y controles o transversales.

3. Los niveles de actividad física deben estar clasificados en bajo, medio o moderado y alto.

4. Los participantes de los estudios deben ser mayores de 18 años y estar aparentemente sanos.

5. Los estudios deben contener datos estadísticos para poder calcular el tamaño del efecto mediante el estadístico RR.

\subsubsection{Extracción de datos}

En principio, se realizó una búsqueda electrónica en la base de datos PUBMED. El período de búsqueda se llevó a cabo entre el 06/01/2014 y se extendió hasta el 07/04/2014. Para ello, se confeccionó una ecuación de búsqueda mediante la utilización descriptores $\mathrm{MeSH}^{4}$ y de operadores booleanos, la cual estuvo constituida por los siguientes términos:

("Motor Activity" AND "Prospective Studies") AND ("Cardiovascular Diseases" OR "Stroke") AND ("Relative Risk")

Los resultados obtenidos fueron 242 estudios, de los cuales se examinaron los resúmenes para determinar si podían llegar a cumplir con los criterios de selección establecidos anteriormente, y poder recuperarlos para decidir acerca de su inclusión.

\footnotetext{
${ }^{4}$ Medical Subject Headings
} 
Por último, se llevo a cabo una búsqueda manual en las referencias bibliográficas de los artículos incluidos para identificar bibliografia que no se haya encontrado en la búsqueda. Analizados los resultados obtenidos en la búsqueda se determinó la inclusión de 19 estudios.

\section{Figura 4. Resultados de Búsqueda en PUBMED}

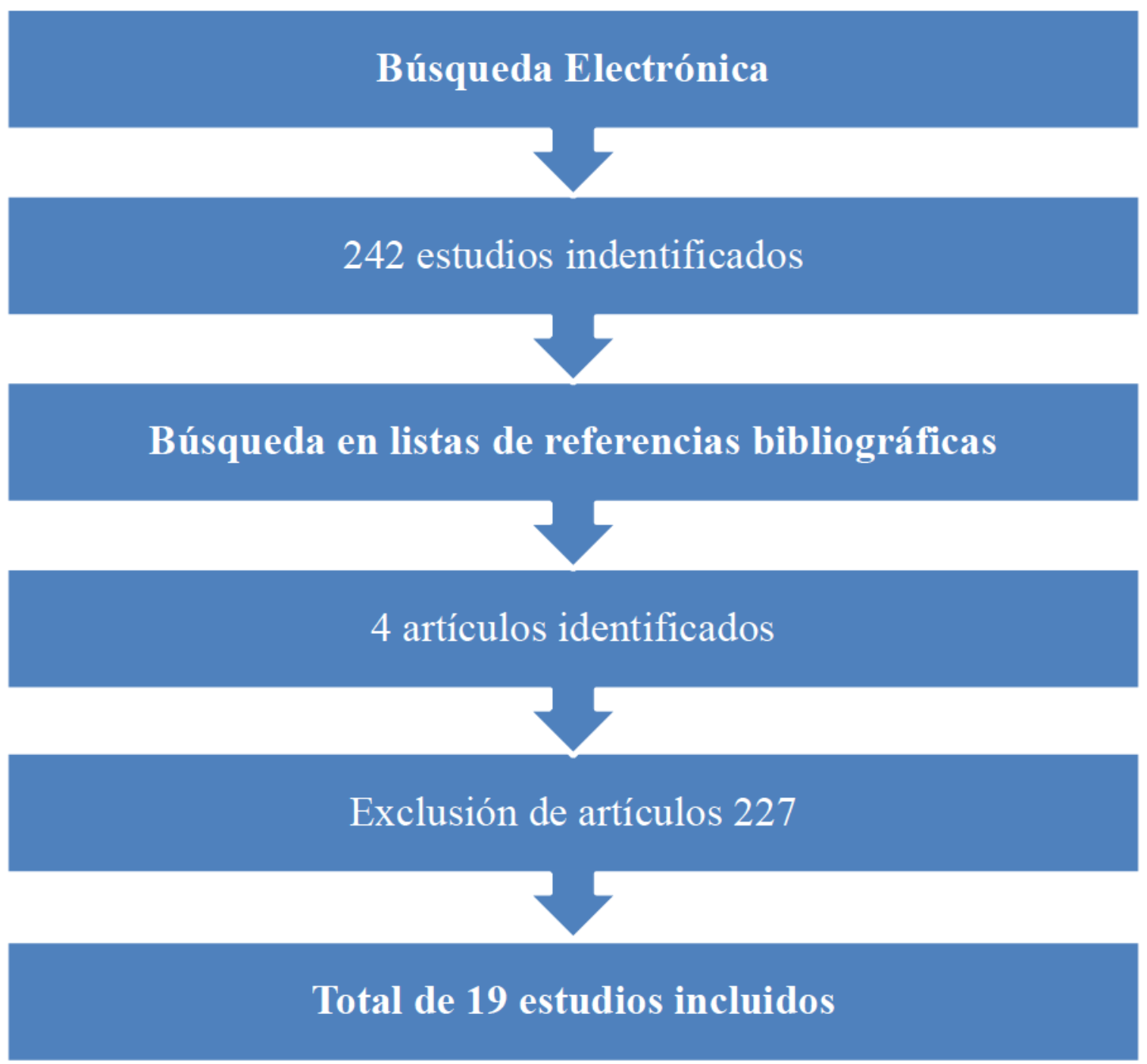

\subsubsection{Codificación de los estudios}

Para realizar la tarea de codificar los estudios incluidos, se dividió a las variables moderadoras de la siguiente forma: 
Figura 5. Codificación de las variables moderadoras

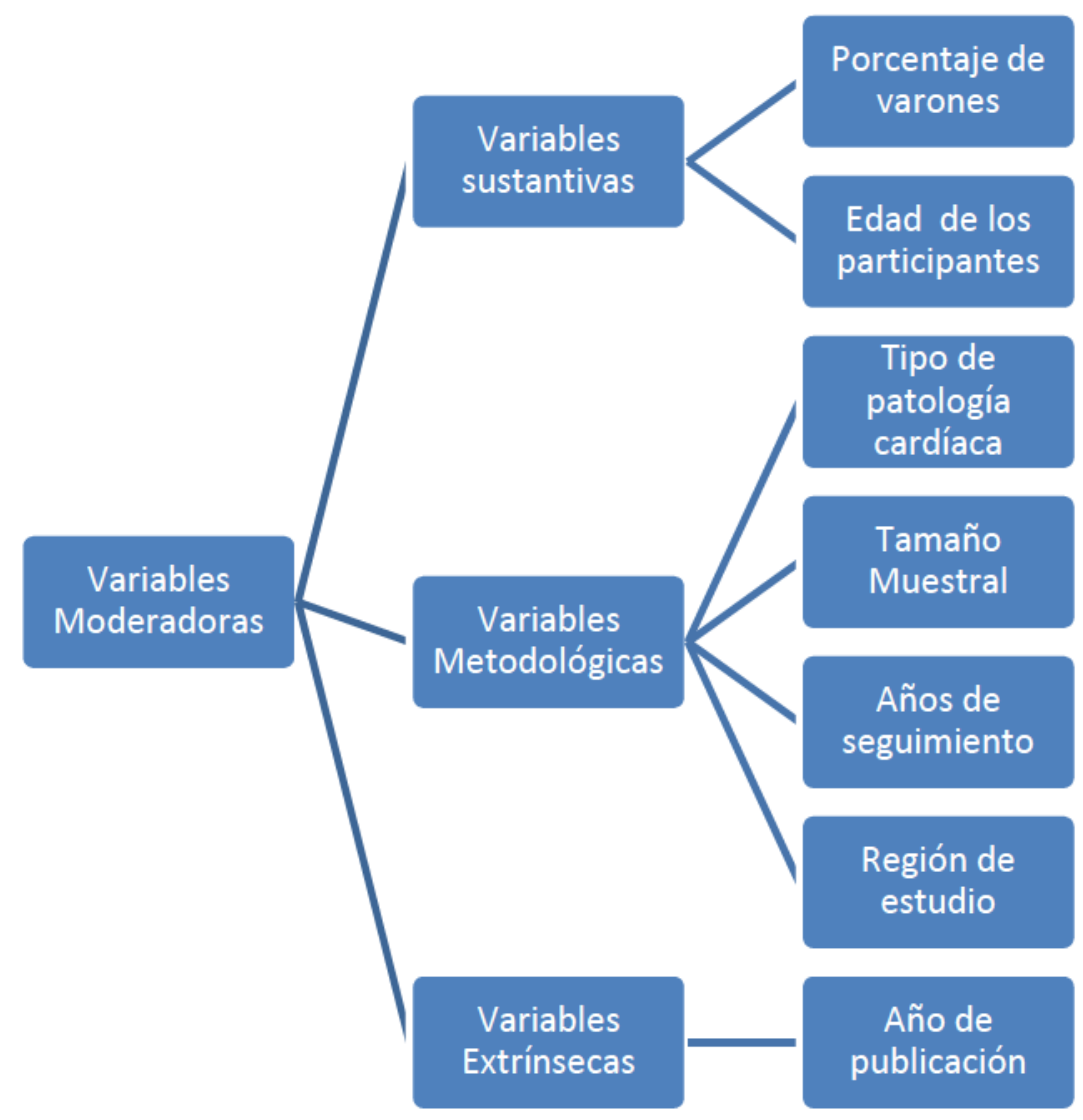

\subsubsection{Variables moderadoras}

1. Variables sustantivas: Las variables sustantivas fueron divididas en:

a. Porcentaje de Varones: se codificó la cantidad de varones de cada estudio en forma porcentual.

b. Edad de los participantes: se codificó la edad media de los participantes de cada estudio.

c. Tipo de patología cardíaca: El tipo de patología cardíaca se codificó de acuerdo a:
i. $1=$ Enfermedad Coronaria
ii. $2=$ Enfermedad Cerebrovascular
iii. $3=$ Enfermedad Coronaria y
Cerebrovascular 


\section{Variables Metodológicas:}

a. Tamaño muestral: El tamaño muestral se codificó de acuerdo al número de participantes que integran el estudio.

b. Años de seguimiento: Se codificó en cantidad de años que fueron seguidos los participantes de cada estudio.

c. Región de estudio: se codificaron las regiones en las que se realizaron los estudios de la siguiente manera:
i. $0=$ Europa
ii. 1=América del Norte
iii. $2=$ Asia

\section{Variables extrínsecas:}

a. Año de publicación: Se codificó el año de publicación de cada estudio medido en años (Ej: 1990).

\subsubsection{Cálculo del tamaño del efecto y análisis estadístico}

Para evaluar la eficacia de la actividad física en la reducción del riesgo cardiovascular se calculó el tamaño del efecto mediante el estadístico de riesgo relativo:

$$
R R=\frac{\frac{a}{n_{e}}}{\frac{b}{n_{c}}}
$$

y el estadístico de Odds Ratio:

$$
O R=\frac{\frac{p_{1}}{\left(1-p_{1}\right)}}{\frac{p_{2}}{\left(1-p_{2}\right)}}=\frac{a \cdot d}{b \cdot c}
$$

Calculado el tamaño del efecto individual, se procedió al cálculo del tamaño del efecto medio mediante la utilización del modelo de efectos aleatorios:

$$
T_{i}=\mu_{\theta}+u_{i}+\varepsilon_{i}
$$


donde se asume $\theta_{i} \sim N\left(\mu_{\theta} ; \tau^{2}\right)$.

Este modelo implica un factor de ponderación para cada estimación del tamaño del efecto por la inversa de la varianza intra-estudio:

$$
w_{i}=\frac{1}{V\left(T_{i}\right)}
$$

y un factor de ponderación por la varianza entre-estudios:

$$
w_{i}^{*}=\frac{1}{\left[V\left(T_{i}\right)+\tau^{2}\right]}
$$

La elección de este modelo se justifica debido a que se asumió que debe contemplarse tanto la variabilidad intra-estudios, como también la variabilidad entre-estudios, pudiendo generalizar los resultados a una población mayor (Sánchez-Meca, MarínMartínez \& Medina, 2006).

Estimado el tamaño del efecto, y en caso de detectar heterogeneidad significativa, se procede a realizar un análisis de variables moderadoras, en el caso de variables moderadoras categóricas, se realiza un análisis de varianza (ANOVA), y en caso de variables moderadoras cuantitativas, una meta-regresión. Con estos análisis se podrá observar cuáles son las variables moderadoras que afectan la homogeneidad del tamaño del efecto. 


\section{RESULTADOS}

\subsection{Análisis descriptivo}

En primer lugar, se presenta un análisis descriptivo de las edades de los participantes tomando como unidad de análisis a cada uno de los estudios incluidos:

Tabla 5. Descripción de la edad media de los participantes por estudio

\begin{tabular}{c|ccccc} 
Variable & Obs & Mean & std. Dev. & Min & Max \\
\hline edadmedia & 19 & 54.96316 & 9.138151 & 43.6 & 77.4
\end{tabular}

En el caso de la edad de los participantes, su media es de $54.96 \pm 9.13$ años, su valor mínimo es de 43.6 años y el máximo de 77.4 años.

Tabla 6. Frecuencia de la edad de los participantes por estudio

\begin{tabular}{r|rrr} 
Elad Media & Freq. & Percent & am. \\
\hline 43.6 & 1 & 5.26 & 5.26 \\
45.5 & 1 & 5.26 & 10.53 \\
46.4 & 1 & 5.26 & 15.79 \\
48.5 & 1 & 5.26 & 21.05 \\
48.7 & 1 & 5.26 & 26.32 \\
49.5 & 2 & 10.53 & 36.84 \\
52.2 & 1 & 5.26 & 42.11 \\
53.2 & 1 & 5.26 & 47.37 \\
53.7 & 1 & 5.26 & 52.63 \\
54.5 & 1 & 5.26 & 57.89 \\
54.6 & 1 & 5.26 & 63.16 \\
55 & 1 & 5.26 & 68.42 \\
56.5 & 1 & 5.26 & 73.68 \\
57.5 & 1 & 5.26 & 78.95 \\
59 & 1 & 5.26 & 84.21 \\
62.2 & 1 & 5.26 & 89.47 \\
76.8 & 1 & 5.26 & 94.74 \\
77.4 & 1 & 5.26 & 100.00 \\
\hline Total & 19 & 100.00 & \\
\hline & & &
\end{tabular}

Como se puede observar en la tabla 6, las edades medias de los estudios son heterogéneas y solo se observa una frecuencia de 2 en la edad media 49.5 años. 
Tabla 7. Descripción del tamaño muestral

\begin{tabular}{c|ccccr} 
Variable & Obs & Mean & std. Dev. & Min & Max \\
\hline tamañomues a & 19 & 22525.63 & 24171.25 & 1564 & 87563
\end{tabular}

La variable moderadora tamaño muestral, presenta una media de $22525.63 \pm 24171.25$ participantes, su valor mínimo es de 1564 y su máximo de 87563 participantes.

\section{Tabla 8. Frecuencia del tamaño muestral}

\begin{tabular}{r|rrr}
$\begin{array}{r}\text { Tamañ } \\
\text { Muestral }\end{array}$ & Freq. & Percent & वm. \\
\hline 1564 & 1 & 5.26 & 5.26 \\
2224 & 1 & 5.26 & 10.53 \\
2512 & 1 & 5.26 & 15.79 \\
5079 & 1 & 5.26 & 21.05 \\
5249 & 1 & 5.26 & 26.32 \\
7735 & 2 & 10.53 & 36.84 \\
9758 & 1 & 5.26 & 42.11 \\
12490 & 2 & 10.53 & 52.63 \\
16327 & 1 & 5.26 & 57.89 \\
21794 & 1 & 5.26 & 63.16 \\
21823 & 1 & 5.26 & 68.42 \\
22191 & 1 & 5.26 & 73.68 \\
32677 & 1 & 5.26 & 78.95 \\
34759 & 1 & 5.26 & 84.21 \\
51529 & 1 & 5.26 & 89.47 \\
72488 & 1 & 5.26 & 94.74 \\
87563 & 1 & 5.26 & 100.00 \\
\hline Total & 19 & 100.00 & \\
& & &
\end{tabular}

La frecuencia de los tamaños muestrales de los estudios incluidos también presenta heterogeneidad entre los mismos.

Tabla 9. Descripción de los años de publicación de los estudios

\begin{tabular}{r|rrrrr} 
Variable & Obs & Mean & Std. Dev. & Min & Max \\
\hline añopublica n & 19 & 2004.421 & 7.049947 & 1989 & 2013
\end{tabular}

En el caso del año de publicación de los estudios, su media es $2004.42 \pm 7.04$ años, su valor mínimo es 1989 y su máximo es 2013. 
Tabla 10. Frecuencia de los años de publicación de los estudios

\begin{tabular}{|c|c|c|c|}
\hline Publicación & Freq. & Percent & dm. \\
\hline 1989 & 1 & 5.26 & 5.26 \\
\hline 1992 & 1 & 5.26 & 10.53 \\
\hline 1998 & 1 & 5.26 & 15.79 \\
\hline 1999 & 2 & 10.53 & 26.32 \\
\hline 2000 & 1 & 5.26 & 31.58 \\
\hline 2002 & 1 & 5.26 & 36.84 \\
\hline 2003 & 2 & 10.53 & 47.37 \\
\hline 2004 & 1 & 5.26 & 52.63 \\
\hline 2006 & 1 & 5.26 & 57.89 \\
\hline 2009 & 1 & 5.26 & 63.16 \\
\hline 2010 & 1 & 5.26 & 68.42 \\
\hline 2011 & 3 & 15.79 & 84.21 \\
\hline 2012 & 2 & 10.53 & 94.74 \\
\hline 2013 & 1 & 5.26 & 100.00 \\
\hline Total & 19 & 100.00 & \\
\hline
\end{tabular}

Respecto de la frecuencia de los años de publicación, se puede observar que la mayor frecuencia se presenta en el año 2011 con 3 estudios, seguida por los años 1999, 2003 y 2012 con una frecuencia de 2 estudios en cada año.

Tabla 11. Descripción de la variable patología cardíaca

Tipo de patologia cardiaca

\begin{tabular}{rrrlr}
\hline & Percentiles & Smallest & & \\
$1 \%$ & 1 & 1 & & \\
$5 \%$ & 1 & 1 & & \\
$10 \%$ & 1 & 2 & Obs & 19 \\
$25 \%$ & 2 & 2 & Sum of Wgt. & \\
& & & & 2.105263 \\
$50 \%$ & 2 & Largest & Mean & .5671309 \\
& & 3 & & .3216374 \\
$75 \%$ & 3 & 3 & Variance & .0468063 \\
$90 \%$ & 3 & 3 & Skewness & 3.14595 \\
$95 \%$ & 3 & 3 & Kurtosis &
\end{tabular}

En el caso del tipo de patología cardíaca, dentro de sus medidas de tendencia central se encuentra, la mediana con un valor de 2 , su media con un valor de $2.10 \pm 0.56$ (Enfermedad Coronaria=1; Enfermedad Cerebrovascular=2; Enfermedad Coronaria y Cerebrovascular=3), con un valor mínimo de 1 y un valor máximo de 3 . 
Tabla 12. Frecuencia de la variable patología cardíaca

\begin{tabular}{|c|c|c|c|c|}
\hline & Tipo de Enfermedad & Freq. & Percent & Cum. \\
\hline \multicolumn{2}{|r|}{ Enfermedad Coronaria } & 2 & 10.53 & 10.53 \\
\hline \multirow{3}{*}{ Enfermedad } & Enfermedad Cerebrovascular & 13 & 68.42 & 78.95 \\
\hline & Coronaria y Cerebrovascular & 4 & 21.05 & 100.00 \\
\hline & Total & 19 & 100.00 & \\
\hline
\end{tabular}

Las enfermedades coronarias muestran una frecuencia de 2, las enfermedades cerebrovasculares una frecuencia de 13, y las enfermedades coronarias y cerebrovasculares una frecuencia de 4 .

Tabla 13. Descripción de la Región de estudio

Región de estudio

\begin{tabular}{rrrrr}
\hline & Percentiles & Smallest & & \\
$1 \%$ & 0 & 0 & & \\
$5 \%$ & 0 & 0 & & 19 \\
$10 \%$ & 0 & 0 & Obs & 19 \\
$25 \%$ & 0 & 0 & Sum of Wgt. & \\
& & & & .6842105 \\
$50 \%$ & 1 & Largest & Mean & .7492686 \\
& 1 & 1 & & .5614035 \\
$75 \%$ & 2 & 2 & Variance & .5660739 \\
$90 \%$ & 2 & 2 & Skewness & 2.05306 \\
$95 \%$ & 2 & & Kurtosis &
\end{tabular}

En el caso del tipo de la región del estudio, dentro de sus medidas de tendencia central se encuentra, la mediana con un valor de 1 , su media con un valor de $0.68 \pm 0.74$ (Europa $=0$; América del Norte=1; Asia=2), con un valor mínimo de 0 y un valor máximo de 2 .

Tabla 14. Frecuencia de la Región del estudio

\begin{tabular}{r|ccr} 
Región de estudio & Freq. & Percent & Cum. \\
\hline Europa & 9 & 47.37 & 47.37 \\
América del Norte & 7 & 36.84 & 84.21 \\
Asia & 3 & 15.79 & 100.00 \\
\hline Total & 19 & 100.00 &
\end{tabular}


En el caso de la región del estudio se puede observar que el $47.36 \%$ de los estudios se realizan en Europa, el 36.84\% en América del Norte, y el 15.78\% en Asia.

Los resultados del meta-análisis se dividieron en los siguientes niveles de actividad física para evaluar los cambios en los tamaños del efecto en diferentes escenarios:

- Bajo vs. Moderado

- Moderado vs. Alto

- Bajo vs. Alto 


\subsection{Resultados de nivel Bajo vs. Moderado}

En este apartado, se evaluaron los cambios en el riesgo relativo de personas sedentarias aparentemente sanas, frente a personas que realizan actividad física con una intensidad moderada ${ }^{5}$. Los resultados obtenidos son los siguientes:

\section{Tabla 15. Resultado de Riesgo Relativo nivel Bajo vs. Moderado}

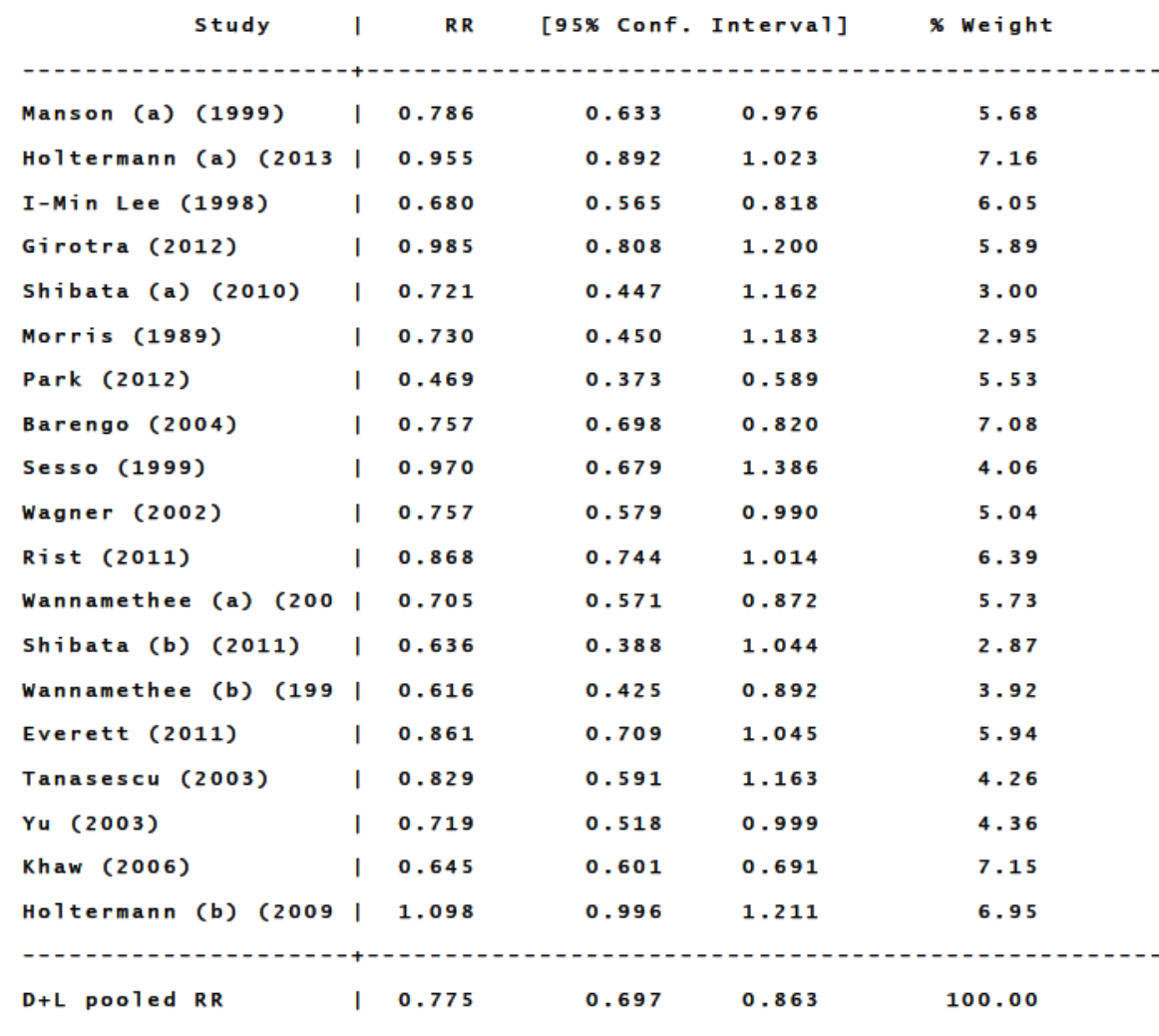

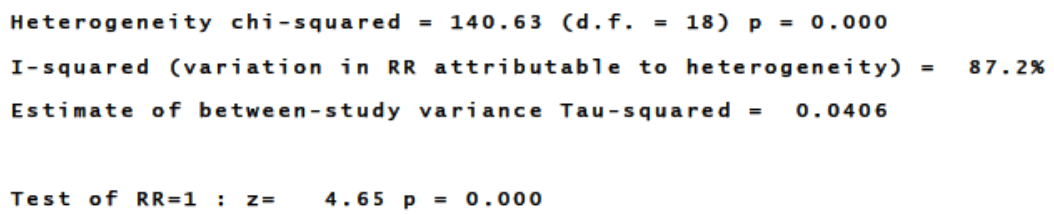

\footnotetext{
${ }^{5}$ Para la comparación de los niveles de actividad física se utilizó la siguiente clasificación en METs:

1. Bajo: 0 a 3 METs

2. Moderado: 3 a 6 METs

3. Alto: $>6$ METs
} 


\section{Figura 6. Forest Plot de riesgo relativo Bajo vs. Moderado}

\section{Bajo vs. Moderado}

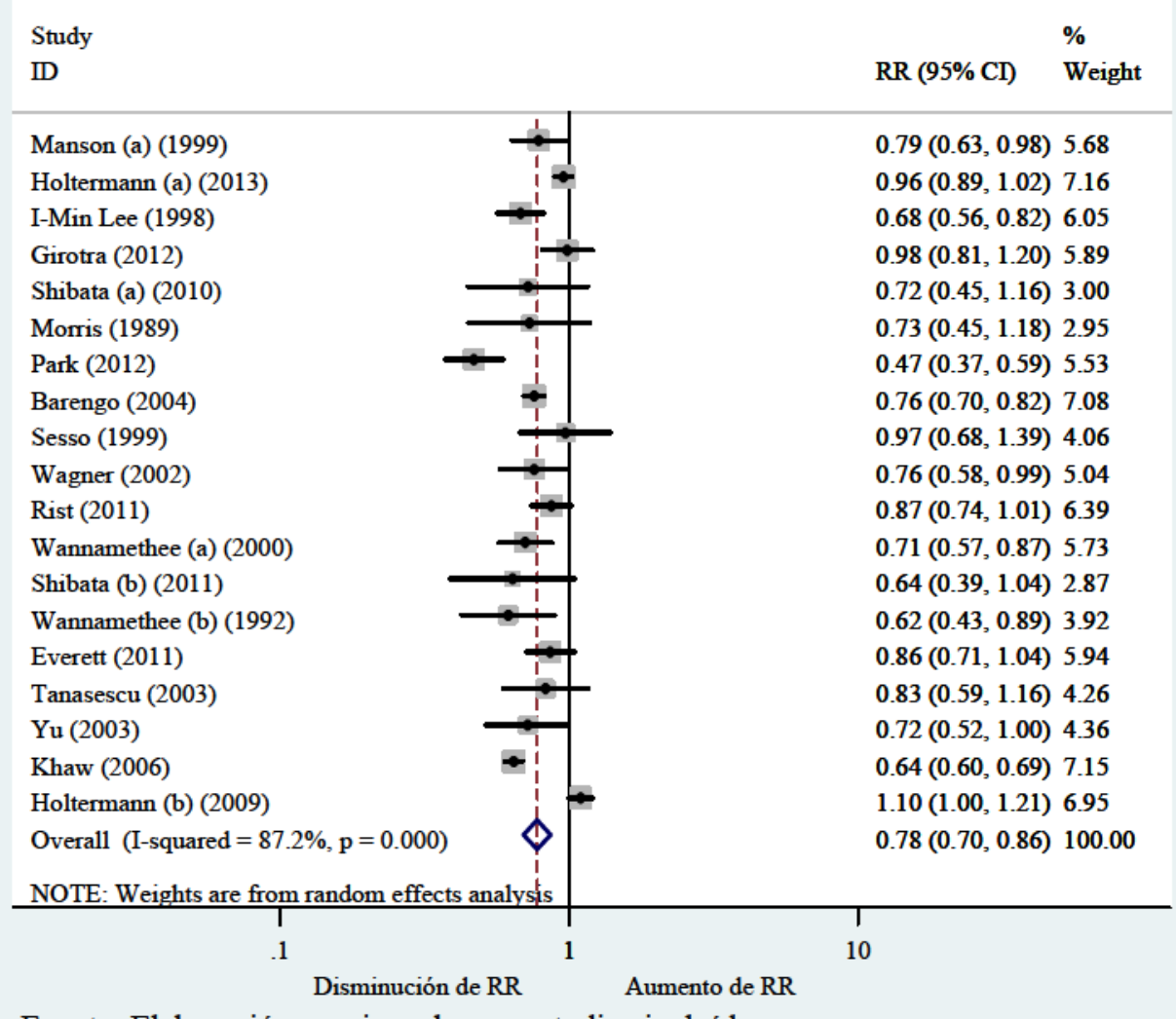

Fuente: Elaboración propia en base a estudios incluídos

En la figura 6 puede observarse una reducción del riesgo relativo $(\mathrm{R} R<1)$ en la mayoría de los estudios con la excepción de uno (Holtermann, 2009). El pooled de los estudios da como resultado $0.78(0.70-0.86)$ con un $p$-valor $=0.000$, el que indica la significatividad de este resultado. Esta estimación indica que las personas que realizan actividad física en niveles moderados en relación con las que realizan actividad física en niveles bajos, disminuyen en un $22 \%$ el riesgo de sufrir enfermedades cardiovasculares.

Para las estimaciones se utilizó el modelo de efectos aleatorios:

$$
T_{i}=\mu_{\theta}+u_{i}+\varepsilon_{i}
$$

donde se asume $\theta_{i} \sim N\left(\mu_{\theta} ; \tau^{2}\right)$. 
Se seleccionó este modelo para contemplarse tanto la variabilidad intra-estudios, así como la variabilidad entre-estudios, pudiendo generalizar los resultados a una población mayor. (Sánchez-Meca, Marín-Martínez \& Medina, 2006)

El estadístico $\mathrm{I}^{2}$ describe el porcentaje de la variación total de los estudios debido a su heterogeneidad (Higgins et. al, 2003). Un valor de $0 \%$ indica que no hay heterogeneidad observada, valores del $25 \%$ al $50 \%$ indican baja heterogeneidad, del $50 \%$ al $75 \%$ heterogeneidad moderada y $>75 \%$ alta heterogeneidad (Higgins \& Thompson, 2004). Valores elevados como los obtenidos en el $\mathrm{RR}\left(\mathrm{I}^{2}=87.2 \%, \mathrm{p}=0.000\right)$ indican que haber utilizado el modelo de efectos fijos sería incorrecto (Harris et. al, 2008). Debido a la heterogeneidad detectada (estadístico $\mathrm{Q}, \mathrm{p}=0.000$ ) posteriormente se realizará un análisis de las variables moderadoras a fin de explicar dicha heterogeneidad.

\subsubsection{Evaluación de sesgo de publicación}

Para evaluar la existencia de un posible sesgo de publicación se realizó un gráfico de embudo (Funnel Plot) el cual analiza el tamaño muestral de cada estudio frente al tamaño del efecto detectado.

\section{Figura 7. Funnel Plot nivel Bajo vs. Moderado}

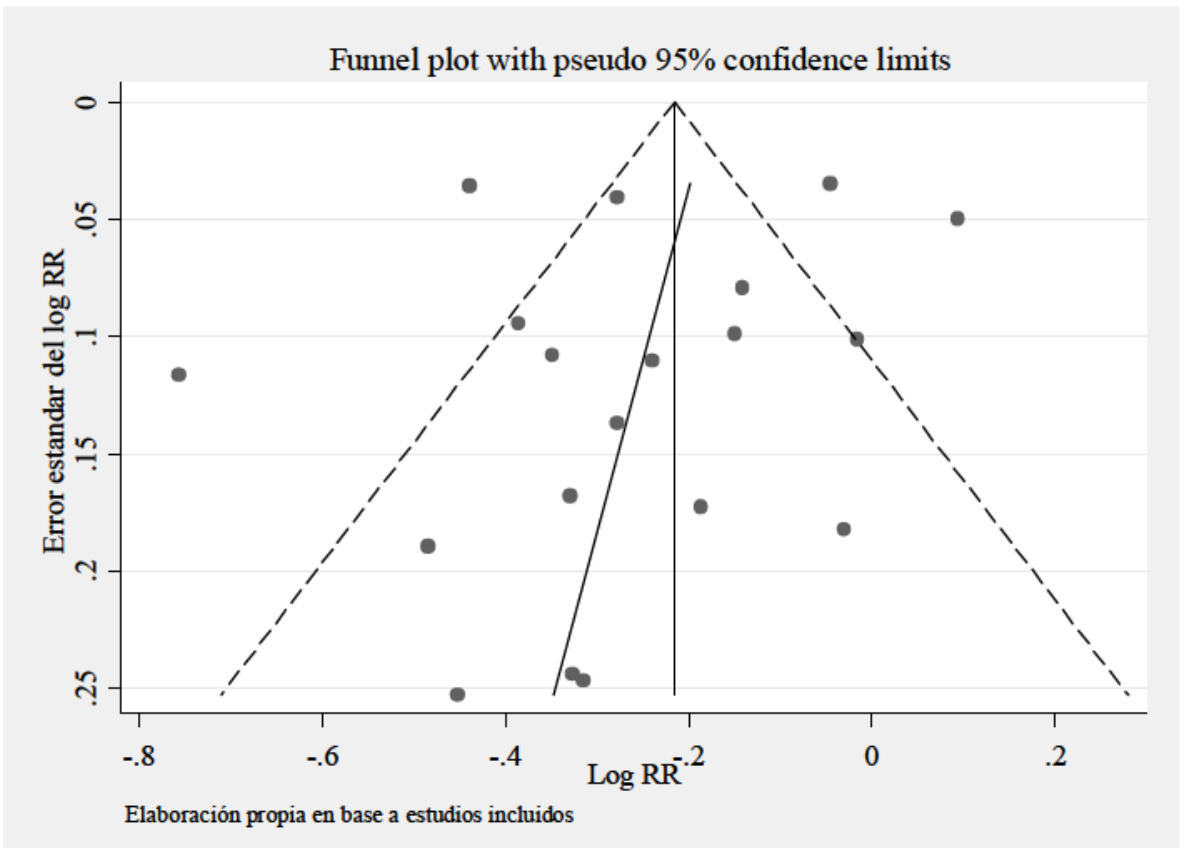


La mayoría de los estudios se encuentran en la V invertida, esto indica cierta asimetría con una leve tendencia hacia la izquierda, no obstante, se identifican 4 estudios en forma asimétrica, esto hace suponer que puede existir algún tipo de sesgo de publicación. Debido a esto, se realizó la prueba de Egger, ya que es más específica y de mayor potencia, sobre todo cuando se cuenta con una cantidad pequeña de estudios. Este test arrojo los siguientes resultados:

\section{Tabla 16. Test Begg sobre sesgo de publicación}

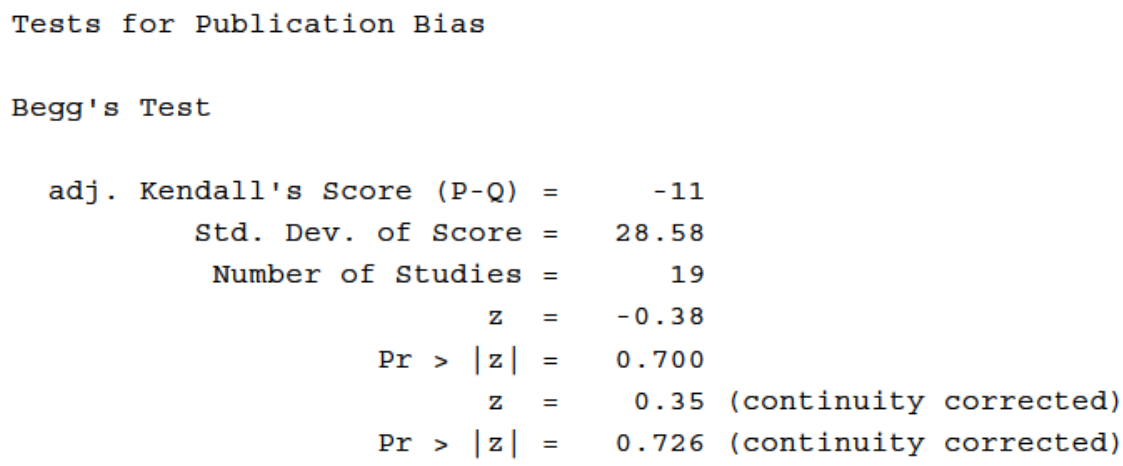

\begin{tabular}{r|rrrrrr}
\hline Std_Eff & Coef. & Std. Err. & $\mathrm{t}$ & $\mathrm{P}>|\mathrm{t}|$ & [95\% Conf. Interval] \\
\hline slope & -.2070179 & .0988602 & -2.09 & 0.052 & -.4155947 & .0015589 \\
bias & -.5223099 & 1.202759 & -0.43 & 0.670 & -3.059909 & 2.01529 \\
\hline
\end{tabular}

\section{Figura 8. Test Egger sobre sesgo de publicación}

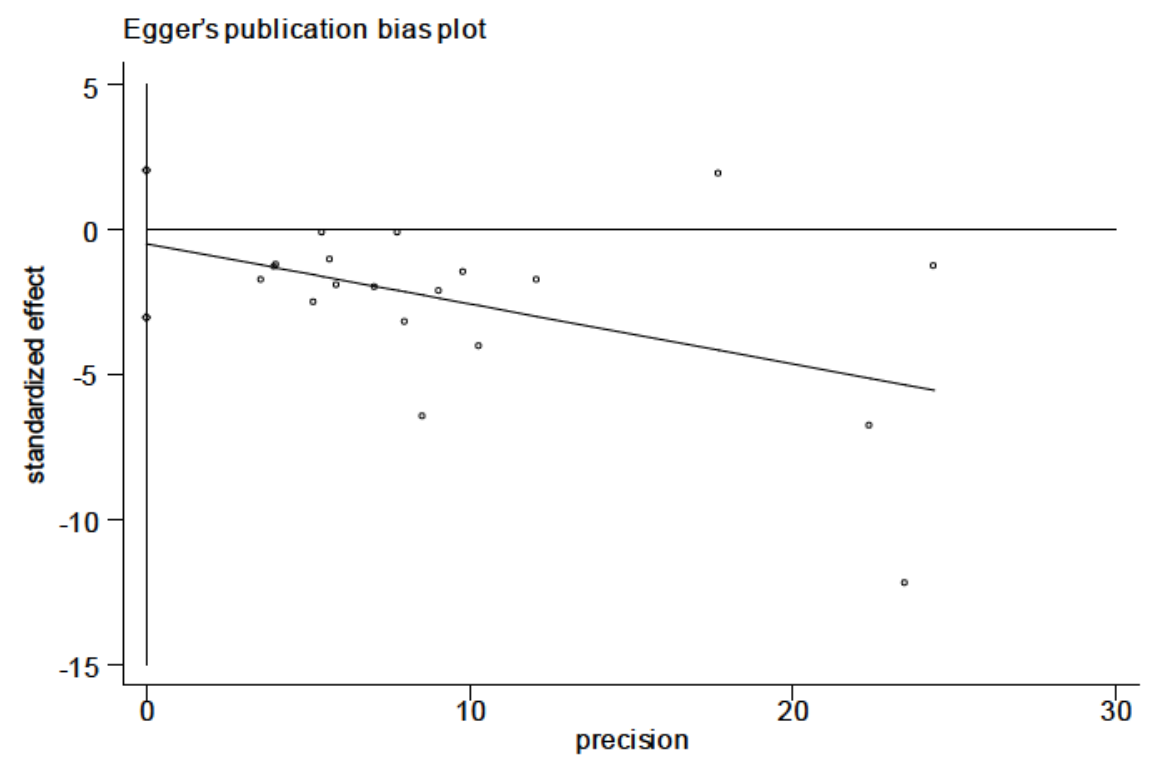


Se puede observar que la ordenada al origen se encuentra cercana a 0 , lo que indica en este test la ausencia de sesgo. Esto se puede confirmar al evaluar el p-valor que arrojo este test, $\mathrm{p}=0.67$ (I.C. 0.95 ), de manera tal que se rechaza la hipótesis nula confirmando la ausencia de sesgo de publicación.

\subsubsection{Análisis de las variables moderadoras}

En este apartado, se analiza cada una de las variables moderadoras con el objetivo de analizar su incidencia en la heterogeneidad del tamaño del efecto. En el caso de variables moderadoras categóricas se realizó un ANOVA, y para las variables moderadoras continuas se realizó una meta-regresión en la que se incluyeron como variable dependiente al logaritmo del tamaño del efecto de cada estudio.

\subsubsection{Variables sustantivas}

La primer variable moderadora a analizar es el porcentaje de varones incluidos en cada uno de los estudios.

Tabla 17. Meta-regresión sobre la variable porcentaje de varones

\begin{tabular}{|c|c|c|c|c|c|c|}
\hline \multicolumn{4}{|c|}{$\begin{array}{l}\text { Meta-regression } \\
\text { REML estimate of between-study variance } \\
\% \text { residua1 variation due to heterogeneity } \\
\text { Proportion of between-study variance explained } \\
\text { With Knapp-Hartung modification }\end{array}$} & \multicolumn{2}{|r|}{$\begin{array}{l}\text { Number of obs } \\
\text { tau2 } \\
\text { I-squared_res } \\
\text { Adj R-squared }\end{array}$} & $\begin{array}{lr}= & 19 \\
= & .03491 \\
= & 86.82 \% \\
= & -5.40 \%\end{array}$ \\
\hline loges & coef. & std. Err. & $t$ & $P>|t|$ & [95\% conf. & Interva1] \\
\hline $\begin{array}{r}\text { orcentaje s } \\
\text { _cons }\end{array}$ & $\begin{array}{r}.0003248 \\
-.2729155\end{array}$ & $\begin{array}{l}.0012897 \\
.0948905\end{array}$ & $\begin{array}{r}0.25 \\
-2.88\end{array}$ & $\begin{array}{l}0.804 \\
0.010\end{array}$ & $\begin{array}{r}-.0023963 \\
-.473117\end{array}$ & $\begin{array}{r}.003045 \\
-.072714\end{array}$ \\
\hline
\end{tabular}

Realizada la meta-regresión se observa un p-valor de 0.804 en un IC del 0.95 , esto indica que esta variable moderadora no presenta significación estadística para explicar la heterogeneidad del tamaño del efecto.

En segundo lugar, se realizó una meta-regresión con la variable moderadora edad media de los participantes, obteniendo los siguientes resultados. 
Tabla 18. Meta-regresión de la variable edad media

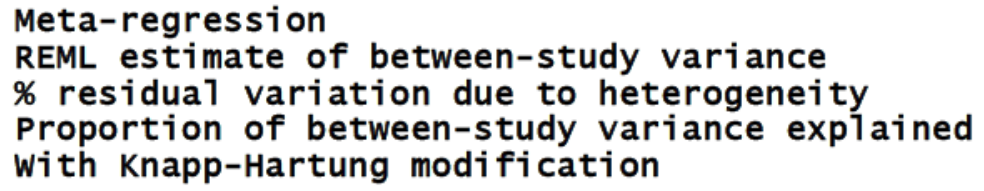

\begin{tabular}{r|rrrrrr}
\hline logES & Coef. & std. Err. & $\mathrm{t}$ & $\mathrm{P}>|\mathrm{t}|$ & [95\% Conf. Interval] \\
\hline edadmedia & -.0075023 & .0053191 & -1.41 & 0.176 & -.0187246 & .0037201 \\
_cons & .1582045 & .2946904 & 0.54 & 0.598 & -.4635379 & .7799469 \\
\hline
\end{tabular}

En este caso, la variable moderadora edad media presenta un p-valor de 0.176 tomando un IC del 0.95 , y un $R^{2}$ ajustado de $7.02 \%$. Estos resultados no indican significación estadística que permita establecer algún tipo de dependencia entre el tamaño del efecto y la edad media de los participantes.

Por último, se realizó un ANOVA sobre la variable moderadora tipo de patología cardíaca con el objetivo de evaluar su influencia en el tamaño del efecto, obteniendo los siguientes resultados:

Tabla 19. Análisis de varianza sobre la variable patología cardíaca

\begin{tabular}{|c|c|c|c|c|c|}
\hline \multirow[b]{2}{*}{ Source } & $\begin{array}{l}\text { Number of obs } \\
\text { Root MSE }\end{array}$ & \multicolumn{2}{|c|}{$\begin{array}{rr}= & 19 \\
= & .220092\end{array}$} & $\begin{array}{l}\text { R-squared } \\
\text { Adj R-squared }\end{array}$ & $\begin{array}{r}=0.0244 \\
=-0.0976\end{array}$ \\
\hline & Partial ss & $\mathrm{df}$ & MS & $\mathrm{F}$ & Prob > F \\
\hline Model & .019355078 & 2 & .00967753 & 0.20 & 0.8209 \\
\hline patologia a & .019355078 & 2 & .00967753 & 0.20 & 0.8209 \\
\hline Residual & .775047069 & 16 & .04844044 & & \\
\hline Total & .794402147 & 18 & .04413345 & & \\
\hline
\end{tabular}

El ANOVA realizado presenta un p-valor de 0.8209 a un 0.95 de IC. Estos resultados no indican significación estadística entre el tipo de patología cardíaca estudiada y el tamaño del efecto. 


\subsubsection{Variables Metodológicas}

En primer lugar, se realizó una meta-regresión de la variable moderadora tamaño muestral, los resultados fueron los siguientes:

Tabla 20. Meta-regresión de la variable tamaño muestral

\begin{tabular}{|c|c|c|c|c|c|c|}
\hline \multicolumn{4}{|c|}{$\begin{array}{l}\text { Meta-regression } \\
\text { REML estimate of between-study variance } \\
\% \text { residual variation due to heterogeneity } \\
\text { Proportion of between-study variance explained } \\
\text { With Knapp-Hartung modification }\end{array}$} & \multicolumn{2}{|r|}{$\begin{array}{l}\text { Number of obs } \\
\text { tau2 } \\
\text { I-squared_res } \\
\text { Adj R-squared }\end{array}$} & $\begin{array}{lr}= & 19 \\
= & .03506 \\
= & 86.78 \% \\
= & -5.87 \%\end{array}$ \\
\hline loges & Coe & std. Err. & $t$ & $\mathrm{t} \mid$ & [95\% Conf. & Interva1 \\
\hline $\begin{array}{r}\text { amañomues a } \\
\text { _cons }\end{array}$ & $\begin{array}{l}-1.72 \mathrm{e}-07 \\
-.2492103\end{array}$ & $\begin{array}{r}2.46 e-06 \\
.074125\end{array}$ & $\begin{array}{l}-0.07 \\
-3.36\end{array}$ & 0. & $\begin{array}{l}-5.35 e-06 \\
-.4056004\end{array}$ & $\begin{array}{r}5.01 \\
-.092\end{array}$ \\
\hline
\end{tabular}

Los resultados de la meta-regresión dieron un p-valor de 0.945 a un IC de 0.95 , en este caso, tampoco se observa significación estadística que permita explicar la heterogeneidad del tamaño del efecto.

En tercer lugar, se procedió a realizar una meta-regresión sobre la variable moderada años de seguimiento de los participantes en cada estudio, arrojando los siguientes resultados:

Tabla 21. Meta-regresión de la variable años de seguimiento

\begin{tabular}{|c|c|c|c|c|c|c|}
\hline \multicolumn{4}{|c|}{$\begin{array}{l}\text { Meta-regression } \\
\text { REML estimate of between-study variance } \\
\% \text { residual variation due to heterogeneity } \\
\text { Proportion of between-study variance explained } \\
\text { With Knapp-Hartung modification }\end{array}$} & \multicolumn{2}{|r|}{$\begin{array}{l}\text { Number of obs } \\
\text { tau2 } \\
\text { I-squared_res } \\
\text { Adj R-squared }\end{array}$} & \multirow{2}{*}{$\begin{array}{lr}= & 19 \\
= & .009467 \\
= & 49.87 \% \\
= & 71.41 \% \\
& \\
\end{array}$} \\
\hline logES & coef. & std. Err. & $t$ & $P>|t|$ & [95\% Conf. & \\
\hline $\begin{array}{r}\text { ñosdesegu o } \\
\text { _cons }\end{array}$ & $\begin{array}{r}.0183901 \\
-.5061069\end{array}$ & $\begin{array}{l}.0040129 \\
.0675737\end{array}$ & $\begin{array}{r}4.58 \\
-7.49\end{array}$ & $\begin{array}{l}0.000 \\
0.000\end{array}$ & $\begin{array}{l}.0099235 \\
-.648675\end{array}$ & $\begin{array}{r}.026856 \\
-.363538\end{array}$ \\
\hline
\end{tabular}

En este caso, se observa un p-valor de 0.00 al 0.95 de IC. Estos resultados indican significación estadística con tendencia positiva entre los años de seguimiento de los participantes y los resultados del tamaño del efecto de cada estudio. Además presenta un $R^{2}$ ajustado que logra explicar un $71.41 \%$ de la heterogeneidad observada. 
En cuarto lugar, se realizó un ANOVA sobre la variable moderadora Región de estudio, dando los siguientes resultados:

Tabla 22. Análisis de varianza sobre la variable Región de estudio

\begin{tabular}{|c|c|c|c|c|c|c|}
\hline \multirow[b]{3}{*}{ Source } & \multirow{2}{*}{$\begin{array}{l}\text { Number of obs } \\
\text { Root MSE }\end{array}$} & $=$ & 19 & \multicolumn{2}{|c|}{ R-squared } & \multirow{2}{*}{$\begin{array}{l}=0.3263 \\
=0.2421\end{array}$} \\
\hline & & $=$. & 73788 & Adj & R-squared & \\
\hline & Partial ss & df & MS & & $\mathrm{F}$ & Prob > F \\
\hline Model & .2340559 & 2 & .11702795 & & 3.87 & 0.0424 \\
\hline Región & .2340559 & 2 & .11702795 & & 3.87 & 0.0424 \\
\hline Residual & .483236548 & 16 & .030202284 & & & \\
\hline Total & .717292448 & 18 & .03984958 & & & \\
\hline
\end{tabular}

El ANOVA arrojó un p-valor de 0.0424 a un IC de 0.95 , lo que indica que existe significación estadística entre la región donde se realizó el estudio y la heterogeneidad del tamaño del efecto. También se observa un $R^{2}$ ajustado de 0.2421 , lo que denota que este modelo logra explicar el $24.21 \%$ de la variabilidad del tamaño del efecto.

Como puede observarse los años de seguimiento y la región de estudio son significativas para explicar la variación del RR. En cuanto a la primera, es lógico que los estudios epidemiológicos que tienen diferentes períodos temporales generen heterogeneidad en el RR, ya que en los estudios con menor duración el observador puede perderse de capturar información sobre los participantes. En segundo lugar, dado que la variabilidad de las regiones donde se han realizado los estudios explica en parte la heterogeneidad del $R R$, puede suponerse que existe algún tipo de práctica de investigación no homogénea.

\subsubsection{Variables Extrínsecas}

Por último, se realizó una meta-regresión con la variable moderadora año de publicación, de la cual se obtienen los siguientes resultados: 
Tabla 23. Meta-regresión de la variable año de publicación

\begin{tabular}{|c|c|c|c|c|c|c|}
\hline $\begin{array}{l}\text { Meta-regressi } \\
\text { REML estimate } \\
\% \text { residual va } \\
\text { Proportion of } \\
\text { With Knapp-Ha }\end{array}$ & $\begin{array}{l}\text { between-s } \\
\text { tion due } \\
\text { tween-stuc } \\
\text { ng modific }\end{array}$ & $\begin{array}{l}\text { udy varian } \\
\text { heterogen } \\
\text { variance } \\
\text { tion }\end{array}$ & ained & & $\begin{array}{l}\text { Number of obs } \\
\text { tau2 } \\
\text { I-squared_res } \\
\text { Adj R-squared }\end{array}$ & $\begin{array}{rr}= & 19 \\
= & .0312 \\
= & 83.60 \% \\
= & 5.79 \%\end{array}$ \\
\hline loges & Coef. & Std. Err. & $t$ & $P>|t|$ & [95\% Conf. & Interva1] \\
\hline $\begin{array}{r}\text { añopub1 ica n } \\
\text { _cons }\end{array}$ & $\begin{array}{r}.00837 \\
-17.03568\end{array}$ & $\begin{array}{l}.0078873 \\
15.81596\end{array}$ & $\begin{array}{r}1.06 \\
-1.08\end{array}$ & $\begin{array}{l}0.303 \\
0.296\end{array}$ & $\begin{array}{l}-.0082706 \\
-50.40444\end{array}$ & $\begin{array}{l}.0250107 \\
16.33308\end{array}$ \\
\hline
\end{tabular}

Se observa un p-valor de 0.303 a un IC de 0.95 y un $R^{2}$ ajustado de $5.79 \%$, según estos resultados la variable moderadora año de publicación no tiene significación estadística para explicar la variabilidad del tamaño del efecto. 


\subsection{Resultados de nivel Moderado vs. Alto}

En este apartado, se evaluaron los cambios en el riesgo relativo de personas aparentemente sanas con un nivel de actividad física moderado, frente a personas con un nivel de actividad física alto ${ }^{6}$. Los resultados son los siguientes:

Tabla 24. Resultado de Riesgo Relativo nivel Moderado vs. Alto

\begin{tabular}{|c|c|c|c|c|}
\hline Study & RR & [95\% Conf. & Interva1] & \% Weight \\
\hline Manson (a) (1999) & I 0.835 & 0.659 & 1.059 & 6.02 \\
\hline Holtermann (a) (2013 & I 1.022 & 0.943 & 1.108 & 7.67 \\
\hline I-Min Lee (1998) & | 1.127 & 0.884 & 1.435 & 5.95 \\
\hline Girotra (2012) & I 0.825 & 0.670 & 1.016 & 6.37 \\
\hline Shibata (a) (2010) & I 0.845 & 0.493 & 1.447 & 3.00 \\
\hline Morris (1989) & I 0.510 & 0.212 & 1.228 & 1.47 \\
\hline Park (2012) & I 0.560 & 0.468 & 0.669 & 6.73 \\
\hline Barengo (2004) & I 0.590 & 0.514 & 0.677 & 7.17 \\
\hline Sesso (1999) & I 0.857 & 0.592 & 1.240 & 4.46 \\
\hline Wagner (2002) & I 1.253 & 0.950 & 1.653 & 5.53 \\
\hline Rist (2011) & I 0.770 & 0.619 & 0.958 & 6.25 \\
\hline Wannamethee (a) (200 & I 0.643 & 0.481 & 0.859 & 5.36 \\
\hline Shibata (b) (2011) & I $\quad 0.679$ & 0.328 & 1.406 & 1.98 \\
\hline Wannamethee (b) (199 & I 0.454 & 0.235 & 0.877 & 2.29 \\
\hline Everett (2011) & I 0.913 & 0.743 & 1.122 & 6.40 \\
\hline Tanasescu (2003) & I 0.711 & 0.484 & 1.044 & 4.31 \\
\hline Yu (2003) & I 0.805 & 0.553 & 1.170 & 4.41 \\
\hline Khaw (2006) & I 0.784 & 0.707 & 0.869 & 7.49 \\
\hline Holtermann (b) (2009 & | 0.991 & 0.861 & 1.140 & 7.14 \\
\hline$D+L$ pooled RR & | 0.806 & 0.717 & 0.907 & 100.00 \\
\hline
\end{tabular}

Heterogeneity chi-squared $=99.42(\mathrm{~d} . \mathrm{f} .=18) \mathrm{p}=0.000$

I-squared (variation in RR attributable to heterogeneity) $=\mathbf{8 1 . 9 \%}$

Estimate of between-study variance Tau-squared $=0.0456$

Test of RR=1: $z=3.58 \mathrm{p}=0.000$

\footnotetext{
${ }^{6}$ Para la comparación de los niveles de actividad física se utilizó la siguiente clasificación en METs:

1. Bajo: 0 a 3 METs

2. Moderado: 3 a 6 METs

3. Alto: $>6 \mathrm{METs}$
} 
Figura 9. Forest Plot de nivel Moderado vs. Alto

\section{Moderado vs. Alto}

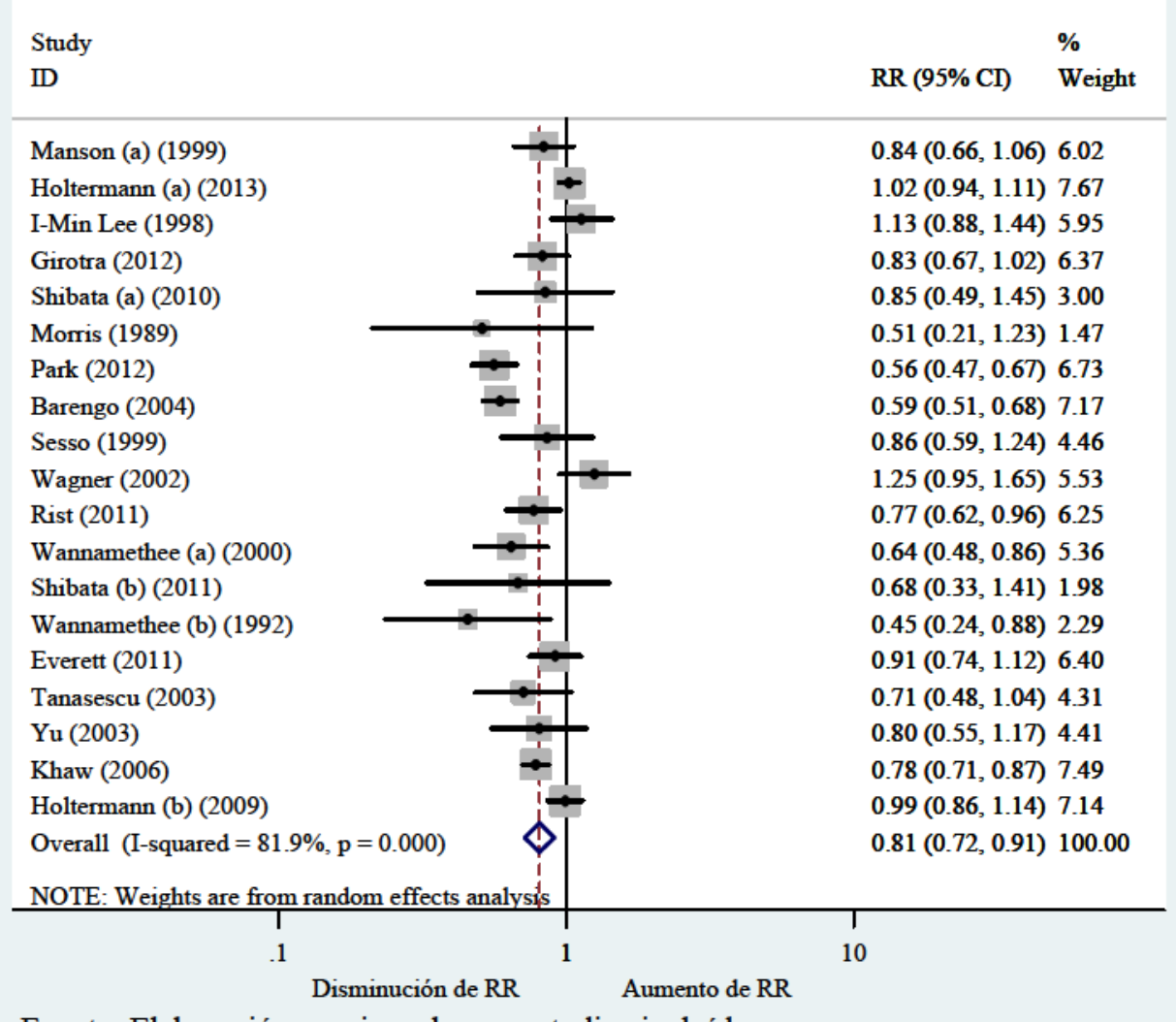

Fuente: Elaboración propia en base a estudios incluídos

En la figura 9 se observa una reducción del riesgo relativo $(\mathrm{RR}<1)$ en la mayoría de los estudios con la excepción de tres (Holtermann, 2013; I-Min Lee, 1998; Wagner, 2002). El pooled de los estudios da como resultado $0.81(0.72-0.91)$ con un $p$-valor $=0.000$ de modo tal que es significativo estadísticamente. Esta estimación indica que las personas que realizan actividad física en niveles altos obtienen una ventaja en cuanto a la reducción del riesgo cardiovascular de un 19\%, en relación con las que realizan actividad física en niveles moderados.

Al igual que para las estimaciones anteriores, se utilizó el modelo de efectos aleatorios:

$$
T_{i}=\mu_{\theta}+u_{i}+\varepsilon_{i}
$$

donde se asume $\theta_{i} \sim N\left(\mu_{\theta} ; \tau^{2}\right)$. 
Se seleccionó este modelo para contemplarse tanto la variabilidad intra-estudios, así como la variabilidad entre-estudios, pudiendo generalizar los resultados a una población mayor (Sánchez-Meca, Marín-Martínez \& Medina, 2006).

El estadístico $\mathrm{I}^{2}$ describe el porcentaje de la variación total de los estudios debido a su heterogeneidad (Higgins et. al, 2003). Un valor de $0 \%$ indica que no hay heterogeneidad observada, valores del $25 \%$ al $50 \%$ indican baja heterogeneidad, del $50 \%$ al $75 \%$ heterogeneidad moderada y $>75 \%$ alta heterogeneidad (Higgins \& Thompson, 2004). Un valor elevado como el obtenido en el $\mathrm{RR}\left(\mathrm{I}^{2}=81.9 \%, \mathrm{p}=0.000\right)$ indica que no hubiera sido adecuado utilizar el modelo de efectos fijos (Harris et. al, 2008). Debido a la heterogeneidad detectada (estadístico $\mathrm{Q}, \mathrm{p}=0.000$ ) posteriormente se realizó un análisis de las variables moderadoras a fin de explicar la heterogeneidad observada.

\subsubsection{Evaluación de sesgo de publicación}

Para evaluar la calidad de los estudios y establecer si existe un sesgo de publicación se realizó un gráfico de embudo (funnel plot), el cual analiza la relación entre el tamaño muestral de los estudios y su tamaño del efecto.

\section{Figura 10. Funnel plot de nivel Moderado vs. Alto}

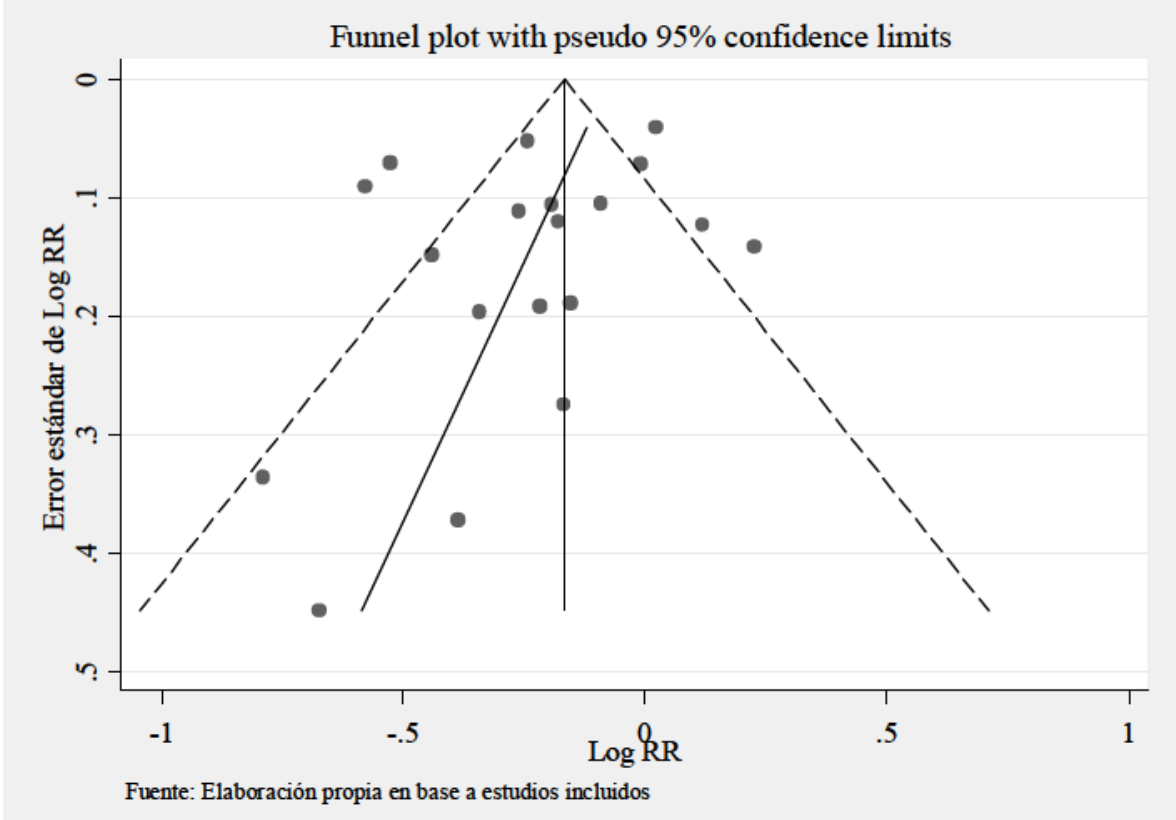


Los resultados del funnel plot indican que existe una asimetría hacia la izquierda con tendencia negativa. Al no ser simétrico el resultado y haber estudios fuera de la $\mathrm{V}$ invertida, debemos realizar una prueba más potente a fin de descartar un sesgo de publicación. Para esto realizamos el test de Egger del cual obtenemos los siguientes resultados:

\section{Tabla 25. Test de Egger para sesgo de publicación}

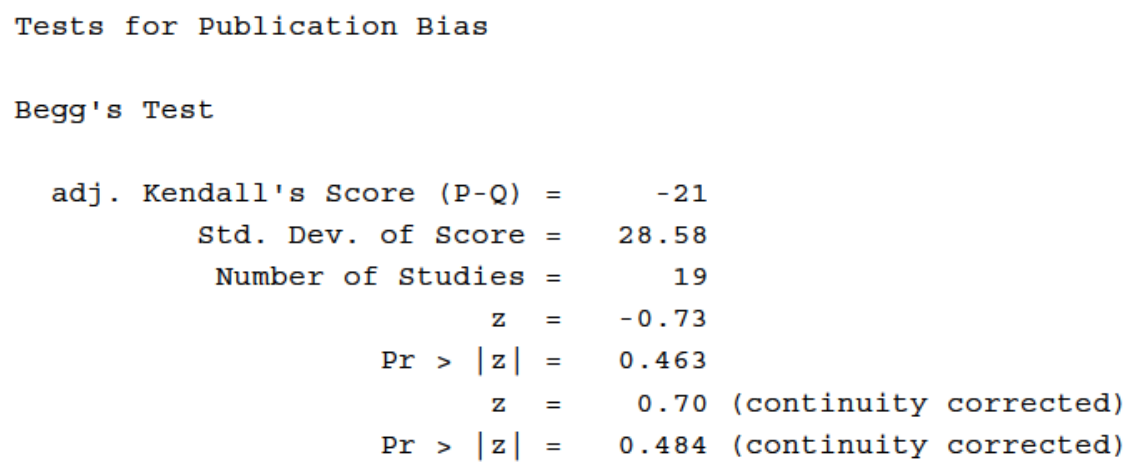

\begin{tabular}{r|rrrrrr}
\hline Std_Eff & Coef. & Std. Err. & $t$ & $\mathrm{P}>|\mathrm{t}|$ & [95\% Conf. Interval] \\
\hline slope & -.10858 & .1098164 & -0.99 & 0.337 & -.3402723 & .1231123 \\
bias & -.916302 & 1.047192 & -0.88 & 0.394 & -3.125684 & 1.29308 \\
\hline
\end{tabular}




\section{Figura 11. Gráfico de Egger para sesgo de publicación}

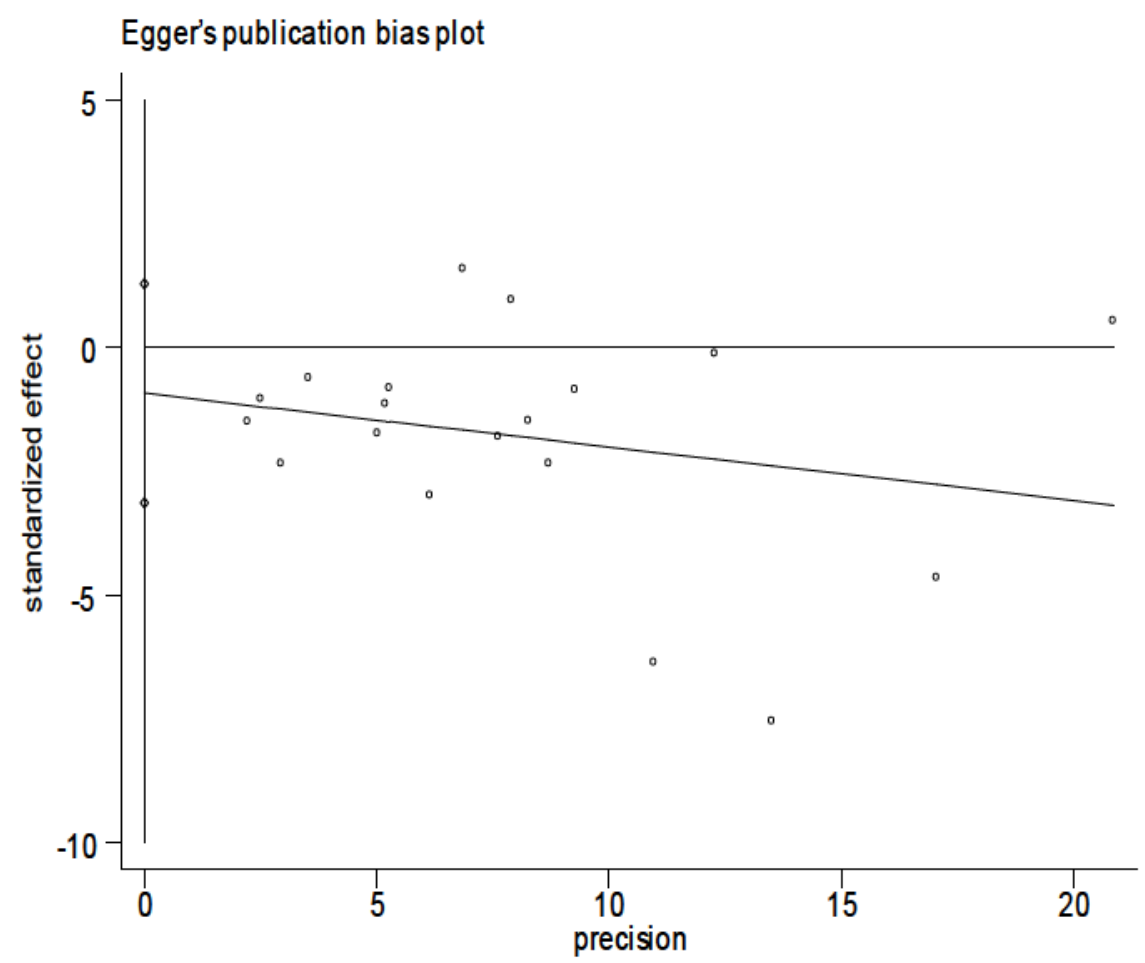

En el gráfico de Egger se observa que la ordenada al origen se encuentra cercana a 0 con lo cual se puede presumir la no existencia de sesgo, sumando a ello observamos que el p-valor del test es igual a 0.394 por lo tanto descartamos la hipótesis nula y por ende desestimamos la presunción de sesgo.

\subsubsection{Análisis de las variables moderadoras}

Debido a que se ha encontrado heterogeneidad en el resultado del tamaño del efecto, se procede a evaluar las variables moderadoras a fin de determinar cuáles podrían explicar esta heterogeneidad.

\subsubsection{Variables sustantivas}

En principio, se procede a realizar una meta-regresión sobre la variable moderadora porcentaje de varones y se obtienen los siguientes resultados: 
Tabla 26. Meta-regresión de la variable porcentaje de varones

\begin{tabular}{|c|c|c|c|c|c|c|}
\hline \multicolumn{5}{|c|}{$\begin{array}{l}\text { Meta-regression } \\
\text { REML estimate of between-study variance } \\
\% \text { residual variation due to heterogeneity } \\
\text { Proportion of between-study variance explained } \\
\text { With Knapp-Hartung modification }\end{array}$} & $\begin{array}{l}\text { Number of obs } \\
\text { tau2 } \\
\text { I-squared_res } \\
\text { Adj R-squared }\end{array}$ & $\begin{array}{lr}= & 19 \\
= & .0416 \\
= & 81.66 \% \\
= & -1.09 \%\end{array}$ \\
\hline $\log E$ & Coef. & d. Er & $t$ & $P>|t|$ & [95\% Conf. & Interval] \\
\hline $\begin{array}{r}\text { orcentaje s } \\
\text { _cons }\end{array}$ & $\begin{array}{r}.000819 \\
-.2621493\end{array}$ & $\begin{array}{l}.0014363 \\
.1021762\end{array}$ & $\begin{array}{r}0.57 \\
-2.57\end{array}$ & $\begin{array}{l}0.576 \\
0.020\end{array}$ & $\begin{array}{l}-.0022114 \\
-.4777222\end{array}$ & $\begin{array}{r}.0038493 \\
-.0465764\end{array}$ \\
\hline
\end{tabular}

Estos resultados muestran un p-valor de 0.57.6 a un IC de 0.95. En función de esto, no se observa significatividad estadística en esta variable moderadora para explicar la heterogeneidad del tamaño del efecto.

Siguiendo con el análisis, se procedió a realizar una meta-regresión sobre la variable moderadora edad media de los participantes para determinar su influencia en la heterogeneidad del tamaño del efecto, los resultados fueron los siguientes:

Tabla 27. Meta-regresión de la variable edad de los participantes

\begin{tabular}{|c|c|c|c|c|c|c|}
\hline $\begin{array}{l}\text { Meta-regressi } \\
\text { REML estimate } \\
\% \text { residual va } \\
\text { Proportion of } \\
\text { with Knapp-Ha }\end{array}$ & $\begin{array}{l}\text { between-s } \\
\text { tion due } \\
\text { tween-stuc } \\
\text { ng modific }\end{array}$ & $\begin{array}{l}\text { ddy varian } \\
\text { heterogen } \\
\text { variance } \\
\text { cion }\end{array}$ & ained & & $\begin{array}{l}\text { Number of obs } \\
\text { tau2 } \\
\text { I-squared_res } \\
\text { Adj R-squared }\end{array}$ & $\begin{array}{rr}= & 19 \\
= & .03955 \\
= & 79.77 \% \\
= & 3.89 \%\end{array}$ \\
\hline loges & coef. & std. Err. & $t$ & $P>|t|$ & [95\% Conf. & Interva1] \\
\hline $\begin{array}{r}\text { edadmedia } \\
\text { _cons }\end{array}$ & $\begin{array}{r}-.0067055 \\
.1550357\end{array}$ & $\begin{array}{r}.005869 \\
.3277542\end{array}$ & $\begin{array}{r}-1.14 \\
0.47\end{array}$ & $\begin{array}{l}0.269 \\
0.642\end{array}$ & $\begin{array}{l}-.0190879 \\
-.5364653\end{array}$ & $\begin{array}{l}.0056769 \\
.8465366\end{array}$ \\
\hline
\end{tabular}

La meta-regresión arrojó un p-valor de 0.269 al 0.95 del IC, y un $R^{2}$ ajustado de $3.89 \%$, en consecuencia, no se observa significación estadística, por lo tanto, la edad media de los participantes no explica la heterogeneidad del tamaño del efecto.

Luego, se realizó un ANOVA sobre la variable moderadora tipo de patología cardíaca con el objetivo de evaluar su influencia en el tamaño del efecto, obteniendo los siguientes resultados: 
Tabla 28. Análisis de varianza de la variable patología cardíaca

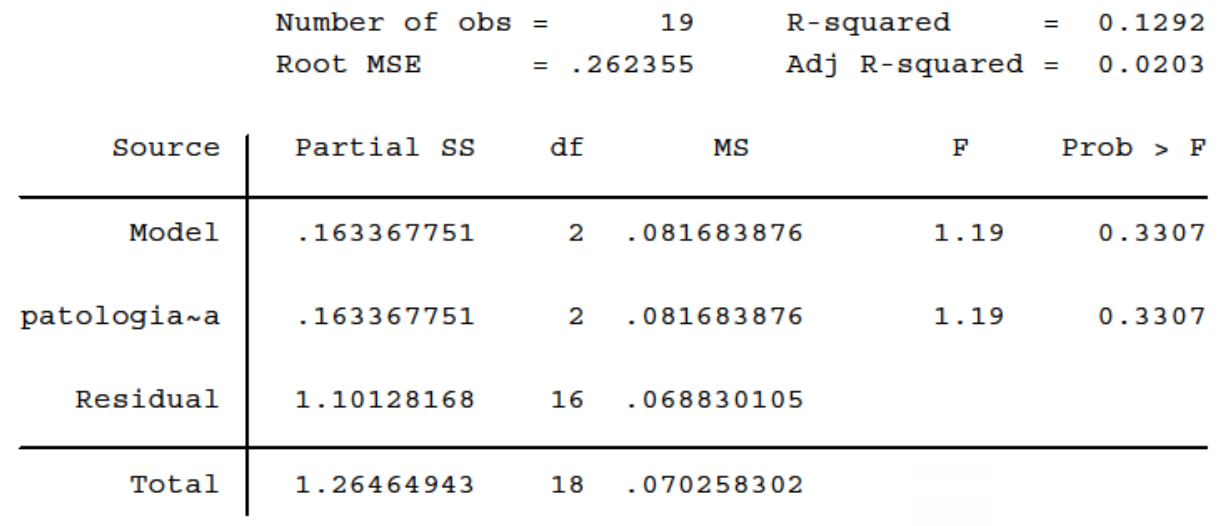

En este caso se observa un p-valor de 0.33 al 0.95 del IC y con un $R^{2}$ ajustado de 0.02 .

De acuerdo a estos resultados no observamos que la variable moderadora patología cardíaca influya en la heterogeneidad del tamaño del efecto.

\subsubsection{Variables Metodológicas}

Se procedió a realizar una meta-regresión sobre la variable moderadora tamaño muestral, de la cual se obtuvieron los siguientes resultados:

Tabla 29. Meta-regresión sobre la variable tamaño muestral

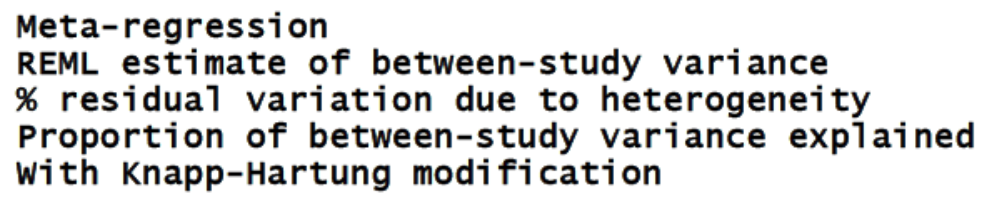

\begin{tabular}{r|rrrrrr}
\hline logES & Coef. & std. Err. & $t$ & $\mathrm{P}>|\mathrm{t}|$ & [95\% Conf. Interva1] \\
\hline tamañomues a & $-1.41 e-06$ & $2.96 e-06$ & -0.48 & 0.641 & $-7.66 \mathrm{e}-06$ & $4.85 \mathrm{e}-06$ \\
_cons & -.1855372 & .0855197 & -2.17 & 0.045 & -.3659679 & -.0051064 \\
\hline
\end{tabular}

Como se puede observar la variable moderadora tamaño muestral no explica la heterogeneidad del tamaño del efecto ya que presenta un p-valor de 0.64 a un 0.95 del IC.

En segundo lugar, se procedió a realizar una meta-regresión para evaluar la variable moderadora años de seguimiento de los participantes y los resultados son los siguientes: 
Tabla 30. Meta-regresión de la variable años de seguimiento

\begin{tabular}{|c|c|c|c|c|c|c|}
\hline \multicolumn{4}{|c|}{$\begin{array}{l}\text { Meta-regression } \\
\text { REML estimate of between-study variance } \\
\% \text { residual variation due to heterogeneity } \\
\text { Proportion of between-study variance explained } \\
\text { With Knapp-Hartung modification }\end{array}$} & \multicolumn{2}{|r|}{$\begin{array}{l}\text { Number of obs } \\
\text { tau2 } \\
\text { I-squared_res } \\
\text { Adj R-squared }\end{array}$} & $\begin{array}{lr}= & 19 \\
= & .03577 \\
= & 71.65 \% \\
= & 13.08 \%\end{array}$ \\
\hline loges & $c$ & d. Er & $\mathrm{t}$ & $P>|t|$ & [95\% Conf. & Interval] \\
\hline $\begin{array}{r}\text { ñosdesegu } 0 \\
\text { _cons }\end{array}$ & $\begin{array}{r}.0102092 \\
-.3571565\end{array}$ & $\begin{array}{r}.0067742 \\
.110595\end{array}$ & $\begin{array}{r}1.51 \\
-3.23\end{array}$ & $\begin{array}{l}0.150 \\
0.005\end{array}$ & $\begin{array}{l}-.0040831 \\
-.5904915\end{array}$ & $\begin{array}{r}.0245016 \\
-.1238214\end{array}$ \\
\hline
\end{tabular}

En este caso, con un p-valor de 0.15 al 0.95 del IC, y con un $R^{2}$ ajustado del $13.08 \%$ no se observa significación estadística, por lo tanto, la variable moderadora años de seguimiento no permite explicar la heterogeneidad del tamaño del efecto.

En tercer lugar, se realizó un ANOVA sobre la variable moderadora región de estudio de la cual se obtuvieron los siguientes resultados:

Tabla 31. Análisis de varianza sobre variable Región de estudio

\begin{tabular}{|c|c|c|c|c|c|c|}
\hline \multirow[b]{3}{*}{ Source } & \multirow{2}{*}{$\begin{array}{l}\text { Number of obs } \\
\text { Root MSE }\end{array}$} & $=$ & 19 & \multicolumn{2}{|c|}{ R-squared } & \multirow{2}{*}{$\begin{array}{l}=0.5624 \\
=0.3436\end{array}$} \\
\hline & & $=.2$ & 14744 & $\operatorname{Adj}$ & R-squared & \\
\hline & Partial ss & df & MS & & $\mathrm{F}$ & Prob > F \\
\hline Model & .71126974 & 6 & .11854495 & & 2.57 & 0.0773 \\
\hline Region & .71126974 & 6 & .118544957 & & 2.57 & 0.0773 \\
\hline Residual & .553379689 & 12 & .046114974 & & & \\
\hline Total & 1. 26464943 & 18 & .070258302 & & & \\
\hline
\end{tabular}

En este caso, el p-valor da 0.07 y el $R^{2}$ ajustado $34.36 \%$, por lo tanto no se encuentra significación estadística y esta variable moderadora tampoco puede explicar la heterogeneidad del tamaño del efecto.

\subsubsection{Variables Extrínsecas}

Por último se realizó una meta-regresión con la variable moderadora año de publicación obteniendo los siguientes resultados: 
Tabla 32. Meta-regresión de la variable año de publicación

\begin{tabular}{|c|c|c|c|c|c|c|}
\hline $\begin{array}{l}\text { Meta-regressi } \\
\text { REML estimate } \\
\% \text { residual va } \\
\text { Proportion of } \\
\text { With Knapp-Ha }\end{array}$ & $\begin{array}{l}\text { between-s } \\
\text { tion due t } \\
\text { tween-stud } \\
\text { ig modific }\end{array}$ & $\begin{array}{l}\text { dy varian } \\
\text { heterogen } \\
\text { variance } \\
\text { ion }\end{array}$ & $\begin{array}{l}y \\
\text { yained }\end{array}$ & & $\begin{array}{l}\text { Number of obs } \\
\text { tau2 } \\
\text { I-squared_res } \\
\text { Adj R-squared }\end{array}$ & $\begin{array}{rr}= & 19 \\
= & .04455 \\
= & 80.93 \% \\
= & -8.26 \%\end{array}$ \\
\hline loges & coef. & std. Err. & $t$ & $P>|t|$ & [95\% conf. & Interval] \\
\hline $\begin{array}{r}\text { añopub1ica n } \\
\text { _cons }\end{array}$ & $\begin{array}{r}.0054057 \\
-11.05701\end{array}$ & $\begin{array}{l}.0101439 \\
20.34546\end{array}$ & $\begin{array}{r}0.53 \\
-0.54\end{array}$ & $\begin{array}{l}0.601 \\
0.594\end{array}$ & $\begin{array}{l}-.0159961 \\
-53.98217\end{array}$ & $\begin{array}{r}.0268074 \\
31.86814\end{array}$ \\
\hline
\end{tabular}

La meta-regresión arrojó y p-valor de 0.60 a un IC de 0.95 . Estos resultados no tienen significación estadística, con lo cual la variable moderadora año de publicación de los estudios no puede explicar la heterogeneidad de los tamaños del efecto. 


\subsection{Resultados de nivel Bajo vs. Alto}

En este apartado, se procedió a evaluar la relación entre el riesgo de enfermedades cardiovasculares y los niveles de actividad física Bajo vs. Alto en personas aparentemente sanas. Los resultados obtenidos son los siguientes:

Tabla 33. Resultado de Riesgo Relativo nivel Bajo vs. Alto

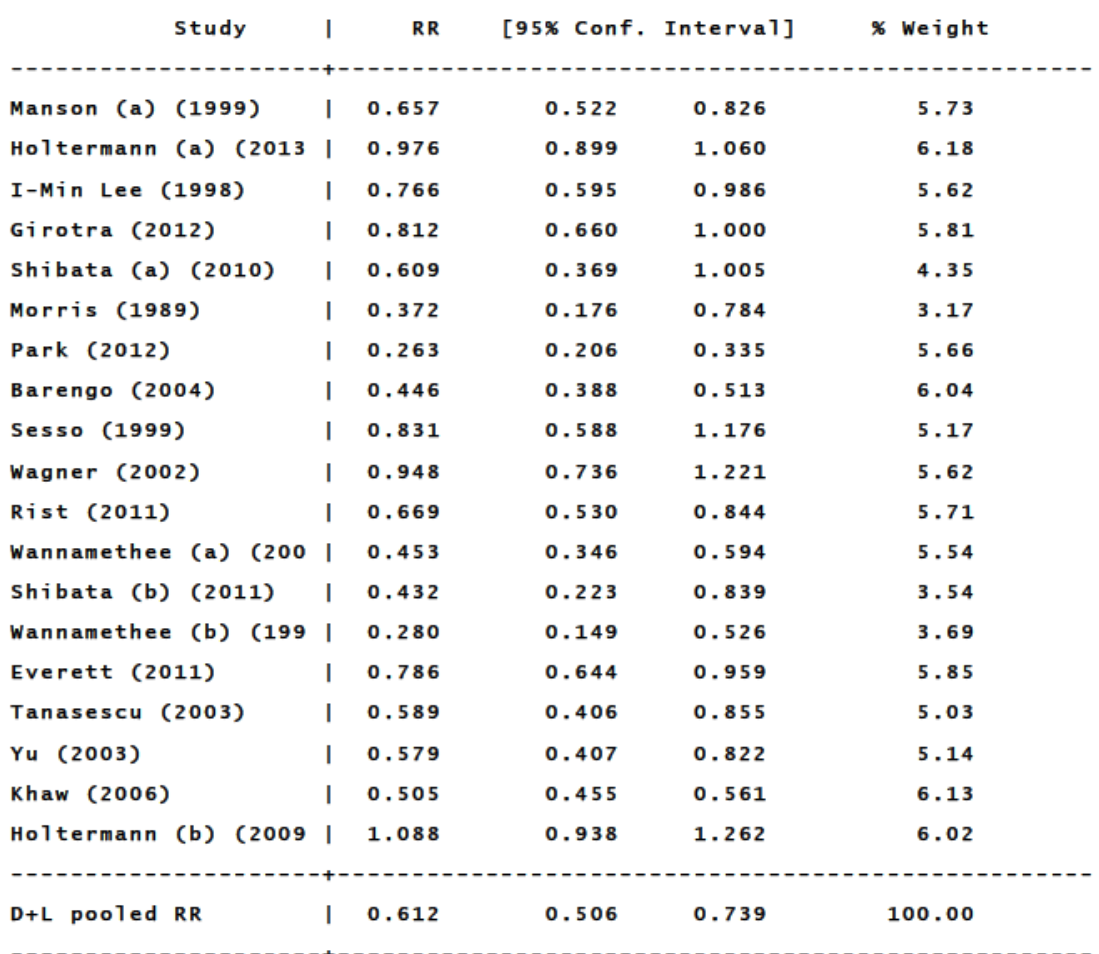

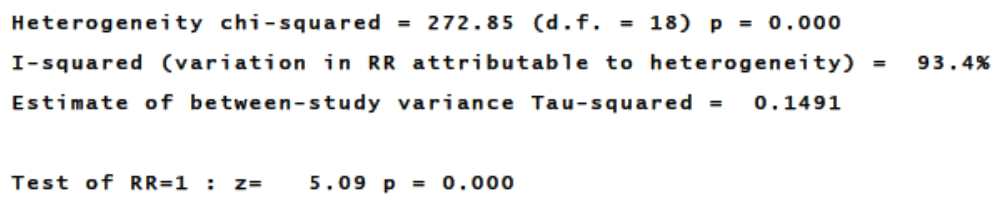




\section{Figura 12. Forest Plot de nivel Bajo vs. Alto}

Bajo vs. Alto

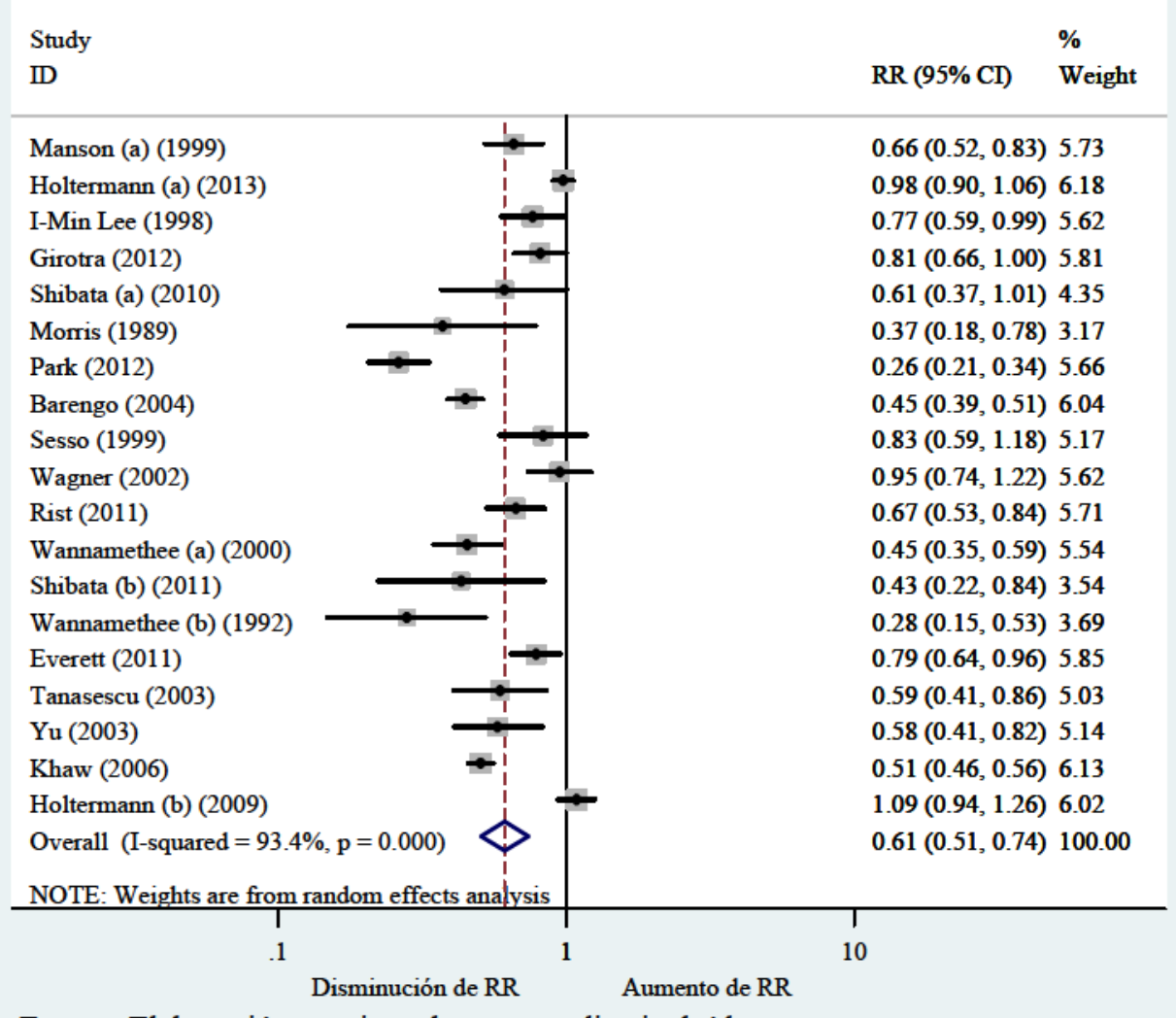

Fuente: Elaboración propia en base a estudios incluídos

En la figura 12 se observa una reducción del riesgo relativo $(\mathrm{R} R<1)$ en la mayoría de los estudios con la excepción de uno (Holtermann, 2009). El pooled de los estudios da como resultado $0.61(0.51-0.74)$ con un $\mathrm{p}$-valor $=0.000$, lo que demuestra que el mismo es significativo estadísticamente. Esta estimación indica que las personas que realizan actividad física en niveles altos obtienen una reducción del riesgo cardiovascular de un $39 \%$ en relación con las que realizan actividad física en niveles bajos.

$\mathrm{Al}$ igual que para las estimaciones anteriores, se utilizó el modelo de efectos aleatorios:

$$
T_{i}=\mu_{\theta}+u_{i}+\varepsilon_{i}
$$

donde se asume $\theta_{i} \sim N\left(\mu_{\theta} ; \tau^{2}\right)$. 
Se seleccionó este modelo para contemplarse tanto la variabilidad intra-estudios, así como la variabilidad entre-estudios, pudiendo generalizar los resultados a una población mayor (Sánchez-Meca, Marín-Martínez \& Medina, 2006).

El estadístico $\mathrm{I}^{2}$ describe el porcentaje de la variación total de los estudios debido a su heterogeneidad (Higgins et. al, 2003). Un valor de $0 \%$ indica que no hay heterogeneidad observada, valores del $25 \%$ al $50 \%$ indican baja heterogeneidad, del $50 \%$ al $75 \%$ heterogeneidad moderada y $>75 \%$ alta heterogeneidad (Higgins \& Thompson, 2004). Un valor elevado como el obtenido en el $R R\left(I^{2}=93.4 \%, p=0.000\right)$ indica que no sería adecuado haber utilizado el modelo de efectos fijos (Harris et. al, 2008). Debido a la heterogeneidad detectada (estadístico Q, p=0.000) posteriormente se realizará un análisis de las variables moderadoras a fin de explicar la heterogeneidad observada.

\subsubsection{Evaluación de sesgo de publicación}

Para evaluar la existencia de un posible sesgo de publicación se realizó un gráfico de embudo (Funnel Plot) el cual analiza el tamaño muestral de cada estudio frente al tamaño del efecto obtenido.

\section{Figura 13. Funnel Plot de nivel Bajo vs. Alto}

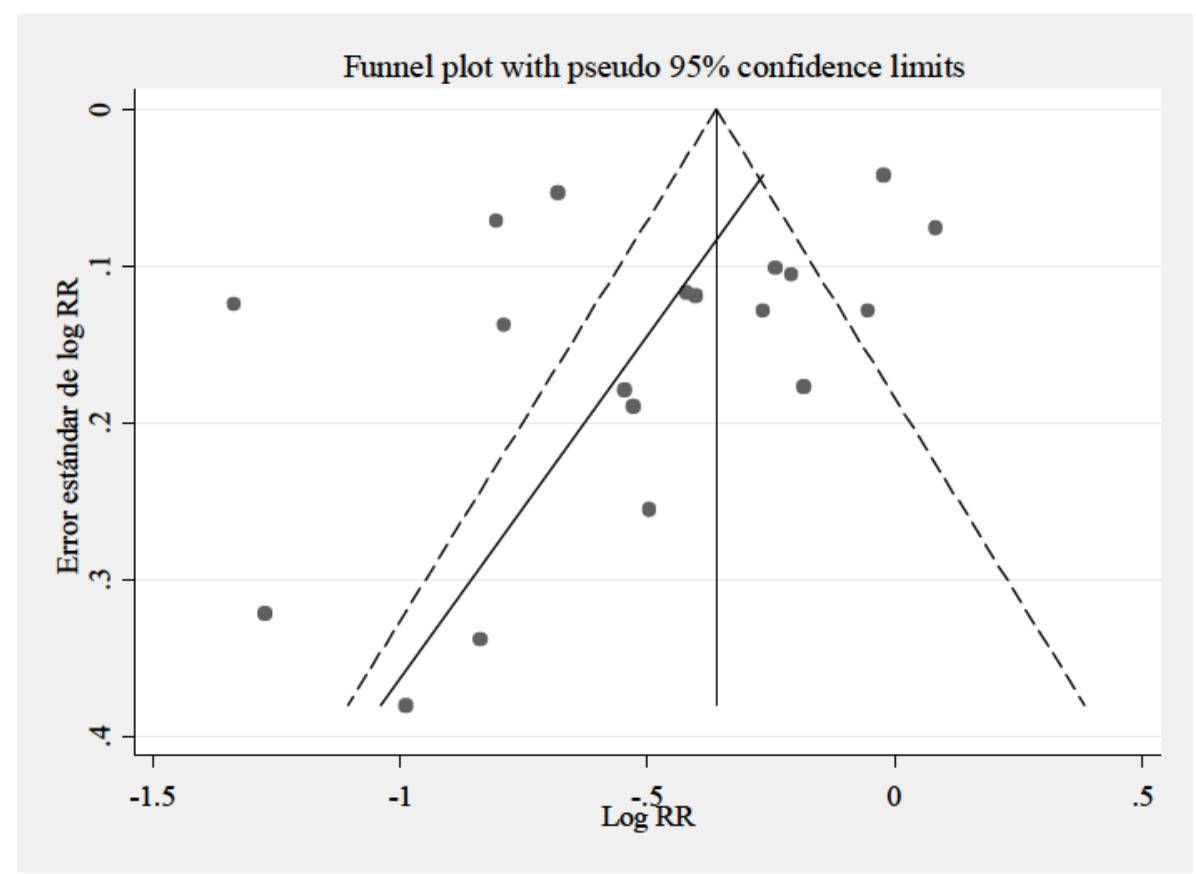


El gráfico indica una asimetría hacia la izquierda dando la posibilidad a la existencia de un sesgo de publicación. Sin embargo, al tratarse de una técnica visual, y por tanto subjetiva, es aconsejable, complementar el estudio de sesgo con pruebas estadísticas más objetivas, como la de Egger (Egger, Smith, Schneider \& Minder, 1997):

\section{Tabla 34. Test de Egger para sesgo de publicación}

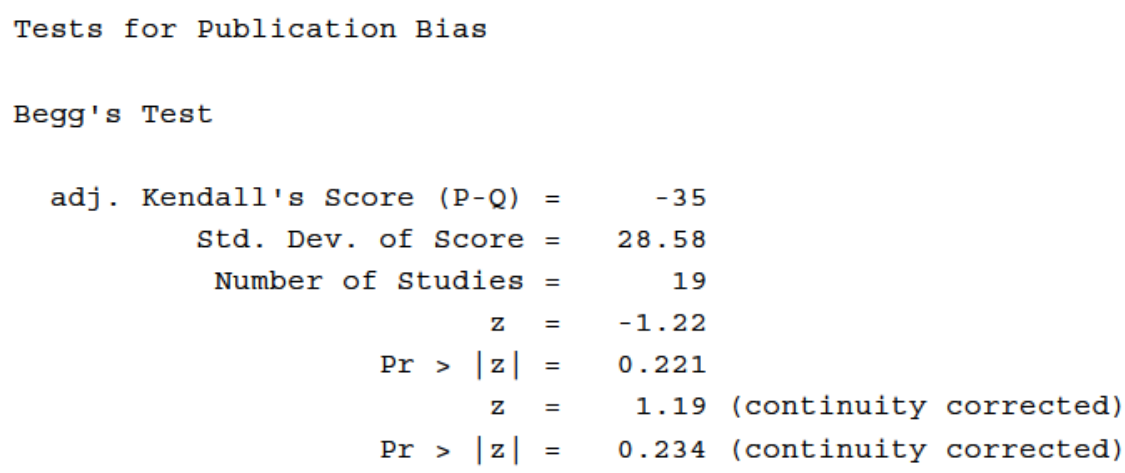

\begin{tabular}{r|rrrrrr}
\hline Std_Eff & Coef. & Std. Err. & $t$ & $\mathrm{P}>|\mathrm{t}|$ & [95\% Conf. Interval] \\
\hline slope & -.23577 & .1934174 & -1.22 & 0.240 & -.6438451 & .1723052 \\
bias & -1.90007 & 1.791952 & -1.06 & 0.304 & -5.680758 & 1.880618 \\
\hline
\end{tabular}

\section{Figura 14. Gráfico de Egger para sesgo de publicación}

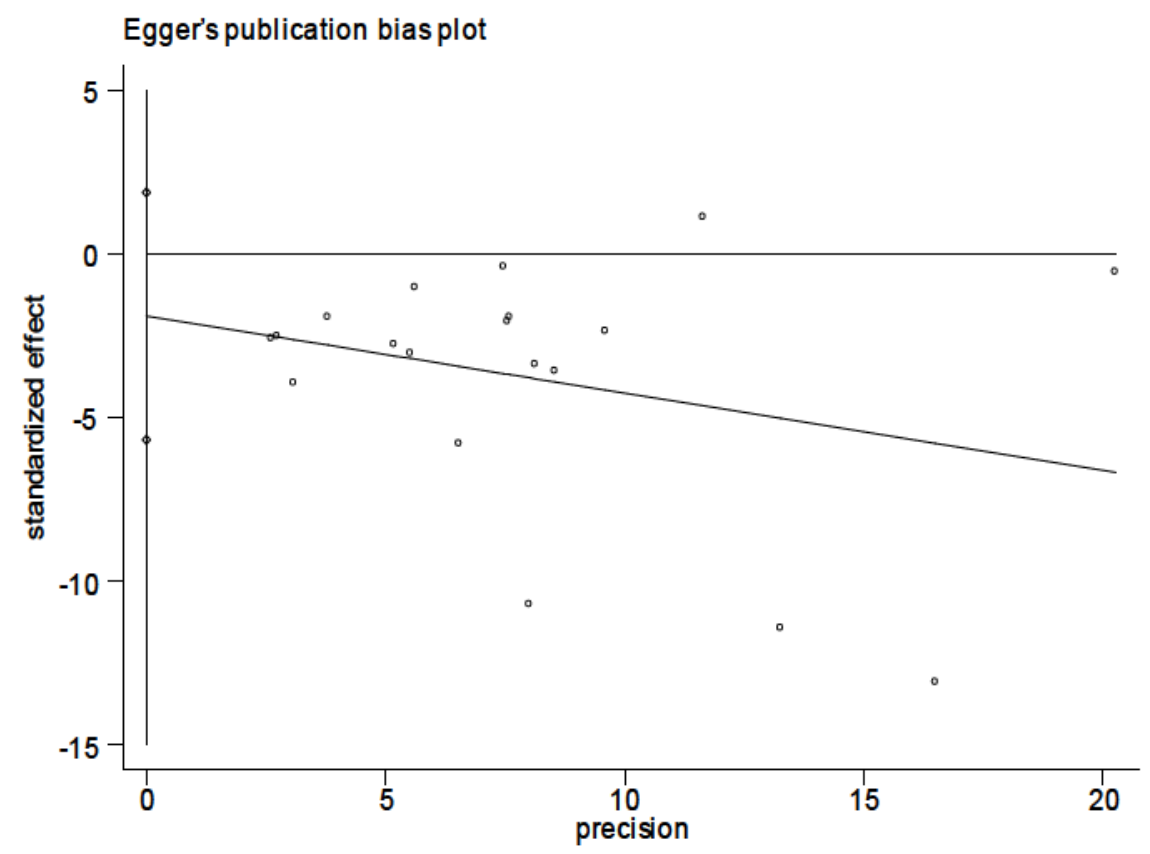


Los resultados del test de Egger muestran un p-valor de 0.304, de esta manera, no se encuentra significación estadística que indique un sesgo de publicación, por otro lado, se puede observar en el gráfico de Egger que la ordenada al origen se encuentra cercana a 0 , lo que también indica ausencia de sesgo de publicación.

\subsubsection{Análisis de las variables moderadoras}

Luego de haber analizado mediante el modelo de efectos aleatorios el tamaño del efecto y obtener significación estadística indicando heterogeneidad, procedemos a evaluar las variables moderadoras con el objetivo de detectar cuales pueden estar influyendo en la heterogeneidad del tamaño del efecto. En el caso de las variables moderadoras categóricas se realizará un análisis de varianza (ANOVA), y las variables moderadoras continuas se someterán a meta-regresiones.

\subsubsection{Variables sustantivas}

En el caso de la variable moderadora porcentaje de varones se realizó una metaregresión obteniendo los siguientes resultados:

Tabla 35. Meta-regresión de la variable porcentaje de varones

\begin{tabular}{|c|c|c|c|c|c|c|}
\hline \multicolumn{4}{|c|}{$\begin{array}{l}\text { Meta-regression } \\
\text { REML estimate of between-study variance } \\
\% \text { residual variation due to heterogeneity } \\
\text { Proportion of between-study variance explained } \\
\text { With Knapp-Hartung modification }\end{array}$} & \multicolumn{2}{|r|}{$\begin{array}{l}\text { Number of obs } \\
\text { tau2 } \\
\text { I-squared_res } \\
\text { Adj R-squared }\end{array}$} & $\begin{array}{lr}= & 19 \\
= & .1398 \\
= & 93.48 \% \\
= & -6.40 \%\end{array}$ \\
\hline loges & coef. & std. Err. & $t$ & $P>|t|$ & [95\% Conf. & Interva1] \\
\hline $\begin{array}{r}\text { orcentaje s } \\
\text { _cons }\end{array}$ & $\begin{array}{r}.0002708 \\
-.5068459\end{array}$ & $\begin{array}{r}.002299 \\
.1696073\end{array}$ & $\begin{array}{r}0.12 \\
-2.99\end{array}$ & $\begin{array}{l}0.908 \\
0.008\end{array}$ & $\begin{array}{l}-.0045796 \\
-.8646859\end{array}$ & $\begin{array}{r}.0051212 \\
-.1490058\end{array}$ \\
\hline
\end{tabular}

De acuerdo a la meta-regresión, se observa un p-valor de 0.90 , por lo tanto, no hay significación estadística que indique que esta variable moderadora pueda explicar la heterogeneidad del tamaño del efecto.

En segundo lugar, se evaluó la variable moderadora edad media de los participantes obteniéndose los siguientes resultados: 
Tabla 36. Meta-regresión de la variable edad media

\begin{tabular}{|c|c|c|c|c|c|c|}
\hline \multicolumn{4}{|c|}{$\begin{array}{l}\text { Meta-regression } \\
\text { REML estimate of between-study variance } \\
\% \text { residual variation due to heterogeneity } \\
\text { Proportion of between-study variance explained } \\
\text { With Knapp-Hartung modification }\end{array}$} & \multicolumn{2}{|r|}{$\begin{array}{l}\text { Number of obs } \\
\text { tau2 } \\
\text { I-squared_res } \\
\text { Adj R-squared }\end{array}$} & $\begin{array}{lr}= & 19 \\
= & .1214 \\
= & 92.409 \\
= & 7.579\end{array}$ \\
\hline logES & $c$ & d. Err & $\mathrm{t}$ & $P>|t|$ & [95\% Conf. & Interval] \\
\hline $\begin{array}{r}\text { edadmedia } \\
\text { _cons }\end{array}$ & $\begin{array}{r}-.0138308 \\
.2721772\end{array}$ & $\begin{array}{l}.0095627 \\
.5322762\end{array}$ & $\begin{array}{r}-1.45 \\
0.51\end{array}$ & $\begin{array}{l}0.166 \\
0.616\end{array}$ & $\begin{array}{l}-.0340062 \\
-.8508273\end{array}$ & $\begin{array}{l}.0063447 \\
1.395182\end{array}$ \\
\hline
\end{tabular}

En este caso, la variable moderadora edad media presenta un p-valor de 0.16 tomando un IC del 0.95 , y un $R^{2}$ ajustado de $7.57 \%$. Estos resultados no indican significación estadística para explicar la heterogeneidad del tamaño del efecto.

En tercer lugar, se realizó un ANOVA sobre la variable moderadora tipo de patología cardíaca con el objetivo de evaluar su influencia en el tamaño del efecto, obteniendo los siguientes resultados:

Tabla 37. Análisis de varianza sobre la variable patología cardíaca

\begin{tabular}{|c|c|c|c|c|c|c|}
\hline \multirow{3}{*}{ Source } & \multirow{2}{*}{$\begin{array}{l}\text { Number of obs } \\
\text { Root MSE }\end{array}$} & \multirow{2}{*}{\multicolumn{2}{|c|}{$\begin{array}{rr}= & 19 \\
= & .410645\end{array}$}} & \multicolumn{2}{|c|}{ R-squared } & \multirow{2}{*}{$\begin{array}{l}=0.0840 \\
=-0.0305\end{array}$} \\
\hline & & & & Adj & R-squared & \\
\hline & Partial ss & df & MS & & $\mathrm{F}$ & Prob > F \\
\hline Model & .247395518 & 2 & .12369775 & & 0.73 & 0.4957 \\
\hline patologia a & .247395518 & 2 & .12369775 & & 0.73 & 0.4957 \\
\hline Residual & 2.69807272 & 16 & .16862954 & & & \\
\hline Total & 2.94546824 & 18 & .16363712 & & & \\
\hline
\end{tabular}

Con un p-valor de 0.49 , no se observa significación estadística que permita explicar la heterogeneidad del tamaño del efecto.

\subsubsection{Variables Metodológicas}

Inicialmente, se realizó una meta-regresión sobre la variable moderadora tamaño muestral, obteniendo los siguientes resultados: 
Tabla 38. Meta-regresión de la variable tamaño muestral

\begin{tabular}{|c|c|c|c|c|c|c|}
\hline $\begin{array}{l}\text { Meta-regressi } \\
\text { REML estimate } \\
\% \text { residual va } \\
\text { Proportion of } \\
\text { With Knapp-Ha }\end{array}$ & $\begin{array}{l}\text { between- } \\
\text { tion due } \\
\text { tween-stu } \\
\text { ig modifi }\end{array}$ & $\begin{array}{l}\text { ly vari } \\
\text { leterog } \\
\text { larianc } \\
\text { on }\end{array}$ & lained & & $\begin{array}{l}\text { Number of obs } \\
\text { tau2 } \\
\text { I-squared_res } \\
\text { Adj R-squared }\end{array}$ & $\begin{array}{rr}= & 19 \\
= & .1381 \\
= & 93.32 \% \\
= & -5.10 \%\end{array}$ \\
\hline loges & Coef. & std. Err. & $t$ & $P>|t|$ & [95\% Conf. & Inte \\
\hline $\begin{array}{r}\text { Imañomues a } \\
\text { _cons }\end{array}$ & $\begin{array}{l}-2.02 \mathrm{e}-06 \\
-.4463704\end{array}$ & $\begin{array}{l}4.30 \mathrm{e}-06 \\
.1314522\end{array}$ & $\begin{array}{l}-0.47 \\
-3.40\end{array}$ & & $\begin{array}{l}-.0000111 \\
-.7237104\end{array}$ & $\begin{array}{r}7.04 \mathrm{e}- \\
-.16903\end{array}$ \\
\hline
\end{tabular}

La meta-regresión con un p-valor de 0.64 al 0.95 del IC, no muestra significación estadística que permita explicar la heterogeneidad del tamaño del efecto.

Luego de ello, se evaluó la variable moderadora años de seguimiento de los participantes, los resultados obtenidos son los siguientes:

Tabla 39. Meta-regresión de la variable años de seguimiento

\begin{tabular}{|c|c|c|c|c|c|c|}
\hline \multicolumn{4}{|c|}{$\begin{array}{l}\text { Meta-regression } \\
\text { REML estimate of between-study variance } \\
\% \text { residual variation due to heterogeneity } \\
\text { Proportion of between-study variance explained } \\
\text { With Knapp-Hartung modification }\end{array}$} & \multicolumn{2}{|r|}{$\begin{array}{l}\text { Number of obs } \\
\text { tau2 } \\
\text { I-squared_res } \\
\text { Adj R-squared }\end{array}$} & $\begin{array}{lr}= & 19 \\
= & .08706 \\
= & 83.66 \% \\
= & 33.73 \%\end{array}$ \\
\hline loges & coef. & std. Err. & $t$ & $P>|t|$ & [95\% conf. & Interva1 \\
\hline $\begin{array}{r}\text { añosdesegu o } \\
\text { _cons }\end{array}$ & $\begin{array}{r}.0274016 \\
-.8595888\end{array}$ & $\begin{array}{l}.0096911 \\
.1550664\end{array}$ & $\begin{array}{r}2.83 \\
-5.54\end{array}$ & $\begin{array}{l}0.012 \\
0.000\end{array}$ & $\begin{array}{l}.0069551 \\
-1.18675\end{array}$ & $\begin{array}{r}.0478481 \\
-.5324273\end{array}$ \\
\hline
\end{tabular}

De acuerdo a la meta-regresión, se observa que esta variable moderadora tiene significación estadística al arrojar un p-valor de 0.012 al 0.95 del IC, por lo tanto, permite explicar la heterogeneidad del tamaño del efecto en un $33.73 \%$.

Por último, se realizó un ANOVA para evaluar el efecto de la variable moderadora región de estudio, obteniendo los siguientes resultados: 
Tabla 40. Análisis de varianza de la variable Región de estudio

\begin{tabular}{|c|c|c|c|c|c|c|}
\hline \multirow[b]{2}{*}{ Source } & $\begin{array}{l}\text { Number of ob } \\
\text { Root MSE }\end{array}$ & $\begin{array}{l}= \\
=.\end{array}$ & $\begin{array}{r}19 \\
4079\end{array}$ & \multicolumn{2}{|c|}{ R-squared } & $\begin{array}{l}=0.7159 \\
=0.5738\end{array}$ \\
\hline & Partial ss & df & MS & & $\mathrm{F}$ & Prob > F \\
\hline Model & 2.10861585 & 6 & .35143597 & & 5.04 & 0.0084 \\
\hline Paisesdes O & 2.10861585 & 6 & .35143597 & & 5.04 & 0.0084 \\
\hline Residual & .836852385 & 12 & .06973769 & & & \\
\hline Total & 2.94546824 & 18 & .16363712 & & & \\
\hline
\end{tabular}

El ANOVA arroja un p-valor de 0.0084 , por lo tanto, existe significación estadística que indica que la variable moderadora región de estudio logra explicar la variabilidad del tamaño del efecto en un 57\%. En función de esto, se puede presumir que las prácticas de investigación aplicadas en distintas regiones difieren en algún sentido, provocando heterogeneidad en el tamaño del efecto.

\subsubsection{Variables Extrínsecas}

Se realizó una meta-regresión con la variable moderadora año de publicación obteniéndose los siguientes resultados:

Tabla 41. Meta-regresión de la variable año de publicación

\begin{tabular}{|c|c|c|c|c|c|c|}
\hline \multicolumn{4}{|c|}{$\begin{array}{l}\text { Meta-regression } \\
\text { REML estimate of between-study variance } \\
\% \text { residual variation due to heterogeneity } \\
\text { Proportion of between-study variance explained } \\
\text { With Knapp-Hartung modification }\end{array}$} & \multicolumn{2}{|r|}{$\begin{array}{l}\text { Number of obs } \\
\text { tau2 } \\
\text { I-squared_res } \\
\text { Adj R-squared }\end{array}$} & $\begin{array}{lr}= & 19 \\
= & .1327 \\
= & 92.03 \% \\
= & -1.04 \%\end{array}$ \\
\hline loges & c & d. Err & $t$ & 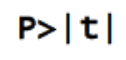 & [95\% Conf. & Interval] \\
\hline $\begin{array}{r}\text { ñopubtica n } \\
\text { _cons }\end{array}$ & $\begin{array}{r}.0155962 \\
-31.75968\end{array}$ & $\begin{array}{l}.0145109 \\
29.09473\end{array}$ & $\begin{array}{r}1.07 \\
-1.09\end{array}$ & $\begin{array}{l}0.297 \\
0.290\end{array}$ & $\begin{array}{r}-.0150191 \\
-93.1442\end{array}$ & $\begin{array}{r}.0462114 \\
29.62484\end{array}$ \\
\hline
\end{tabular}

La meta-regresión muestra un p-valor no significativo de 0.29 al $95 \%$ de IC, en consecuencia, esta variable no logra explicar la heterogeneidad del tamaño del efecto. 


\section{CAPÍTULO IV}

\section{Discusión}

A fin de realizar el meta-análisis y resolver el problema de análisis propuesto, se formularon las siguientes preguntas:

¿Es eficaz la práctica de actividad física para reducir el riesgo de padecer enfermedades cardiovasculares?

¿Cuál es el porcentaje de reducción del riesgo relativo que proporciona la práctica de actividad física en enfermedades cardiovasculares?

¿Existen diferencias en la reducción del riesgo relativo entre los niveles de actividad física Bajo, Moderado y Alto?

Para responder a estar preguntas y como base para realizar este meta-análisis se establecieron los siguientes objetivos:

- Verificar la relación entre la práctica de actividad física y la disminución del riesgo relativo de padecer enfermedades cardiovasculares en estudios de cohortes.

- Diferenciar la modificación del riesgo relativo entre diferentes niveles de actividad física (Bajo, Moderado, Alto).

De acuerdo a la búsqueda bibliográfica se identificaron 246 estudios. Luego de un análisis exhaustivo, se concluyó que un total de 19 estudios cumplían con los criterios de inclusión.

En la primer etapa, se analizaron los niveles de actividad física Bajo vs. Moderado utilizando el modelo de efectos aleatorios. De acuerdo a este análisis, se obtuvo un tamaño del efecto medio de $0.78(0.70-0.86 ; 95 \%$ IC) con un p-valor $=0.000$, lo que indica que es significativo estadísticamente. Luego para evaluar y descartar la posibilidad de un sesgo de publicación se realizó el test de Egger el cual arrojó un pvalor de 0.67 (I.C. 0.95), de esta manera que se confirmó la ausencia de sesgo de publicación. Por último, debido a la presencia de heterogeneidad, se procedió a realizar 
un análisis de las variables moderadoras, con el cual se determinó que los años de seguimiento ( $\mathrm{p}=0.000$; 95\% IC) explican un $71.41 \%$ de la variabilidad del tamaño del efecto y la región de estudio ( $\mathrm{p}=0.0424 ; 95 \%$ IC) explicaba un $24.21 \%$ de la variabilidad del TE.

Estos resultados indican que las personas que realizan actividad física en forma moderada tienen menor riesgo de padecer enfermedades cardiovasculares en comparación con las personas inactivas o que realizan actividad física en un nivel bajo.

Los resultados de este análisis son similares a los obtenidos en un estudio meta-analítico realizado por Li (2012), donde se observó un fuerte efecto protector de la actividad física en un nivel moderado tanto en hombres ( $R R=0,89 ; 95 \%$ IC; $0,82-0,97 ; p=0,008)$ como en mujeres $(\mathrm{RR}=0,83 ; 95 \% \mathrm{IC} ; 0,67-1,03 ; \mathrm{p}=0,089)$. Es importante mencionar que cuatro de los estudios incluidos (Manson, 1999; Wannamethee, 1992; Lee, 1998; Sesso, 1999) también fueron utilizados por Li (2012), en consecuencia el $21.05 \%$ de los estudios incluidos en esta tesis afecta la comparación de los TE.

Otro meta-análisis, analizó los efectos de la actividad física moderada frente a la inactividad en el ámbito laboral y en el tiempo libre, en relación con los accidentes cerebrovasculares, y mostró un efecto protector para los individuos que realizaban actividad física en el trabajo $(\mathrm{RR}=0,64 ; 95 \% \mathrm{IC} ; 0,48-0,87)$ así como también para los que realizaban actividad física en el tiempo libre $(\mathrm{RR}=0,85,95 \% \mathrm{IC} ; 0,78-0,93)$ (Wendel-Vos et. al, 2004). De los estudios incluidos en ese meta-análisis, dos de ellos fueron seleccionados para esta tesis (Wannamethee, 1992; Lee 1998), en función de esto el $10.5 \%$ de los estudios afecta la comparación del TE.

Por último, Blair (2003) realizó un meta-análisis donde observó a individuos moderadamente activos en comparación con personas inactivas, en relación con accidentes cerebrovasculares isquémicos y hemorrágicos. En este estudio, se encontraron resultados similares entre los individuos moderadamente activos frente a los inactivos $(\mathrm{RR}=0,83$ en estudios de cohorte; $\mathrm{R}=0,52$ para estudios de casos $\mathrm{y}$ controles; $\mathrm{RR}=0,80$ para los dos combinados). En este caso, dos de los estudios seleccionados por Blair (Wannamethee, 1992; Lee, 1998) fueron incluidos en esta tesis, por lo tanto, el $10.5 \%$ de los estudios afectan la comparación del TE. 
En la segunda etapa, se analizaron los niveles de actividad física Moderado vs. Alto utilizando el modelo de efectos aleatorios. En este análisis, se observó un tamaño del efecto medio de $0.81(0.72-0.91 ; 95 \%$ IC) con un $\mathrm{p}$-valor $=0.000$ lo que indica significatividad estadística. Posteriormente, a fin de evaluar y descartar la posibilidad de un sesgo de publicación se realizó el test de Egger, con el que se observó un p-valor de 0.394 (I.C. 0.95), con lo que se confirmó la ausencia de un posible sesgo de publicación. Finalmente y debido a la presencia de heterogeneidad, se procedió a realizar un análisis de las variables moderadoras, mediante el cual no se obtuvo significatividad estadística en ninguna de las variables y por ende no se pudo explicar la heterogeneidad detectada.

De acuerdo a los resultados obtenidos, se puede asumir que las personas que realizan actividad física con una intensidad alta tienen un menor riesgo de enfermedades cardiovasculares en relación con las personas que realizan actividad física en forma moderada. Para comparar estos resultados podemos citar el meta-análisis realizado por Wendel-Vos (2004), en donde se observó que altos niveles de actividad física en el trabajo tienen un efecto protector contra accidentes cerebrovasculares, en relación con niveles de actividad física moderados ( $\mathrm{R}=0.77$; 95\% IC; 0.60-0.98), así como también los niveles altos de actividad física en el tiempo libre proveen un efecto protector contra accidentes cerebrovasculares en relación con niveles moderados $(\mathrm{RR}=0.84 ; 95 \% \mathrm{IC}$; $0.63-1.11)$.

En la tercer etapa, se analizaron los niveles de actividad física Bajo vs. Alto utilizando el modelo de efectos aleatorios. Se obtuvo un tamaño del efecto medio de 0.61 (0.51$0.74 ; 95 \%$ IC) con un p-valor $=0.000$ que indica significatividad estadística. Para descartar un sesgo de publicación, se procedió a realizar el test de Egger obteniéndose un p-valor de 0.304, lo que indica ausencia de sesgo. Al haberse encontrado heterogeneidad en los resultados, se realizó un análisis de las variables moderadoras. De este análisis se desprendió que, la variables años de seguimiento $(\mathrm{p}=0.012 ; 0.95 \%$ IC) explican un $33.73 \%$ de la variabilidad del TE y la región de estudio ( $\mathrm{p}=0.0084 ; 95 \%$ IC) explica un $57 \%$ de la variación del TE.

Los resultados de este análisis indican que las personas que realizan actividad física con intensidades altas en relación con los individuos que la realizan con una intensidad baja, tienen un menor riesgo frente a las enfermedades cardiovasculares. Para comparar 
nuestros resultados, podemos citar el meta-análisis realizado por Li (2012), en donde se encontró una asociación positiva entre intensidades altas de actividad física en comparación con intensidades bajas y la disminución del riesgo de enfermedades cardiovasculares, tanto en hombres que realizan actividad física en su trabajo $(R R=0.91$; 0.84-0.97; 95\% IC) como en mujeres ( $\mathrm{RR}=0.84 ; 0.77-0.92 ; 95 \%$ IC), así como también en hombres que realizan actividad física en su tiempo libre $(\mathrm{RR}=0.76 ; 0.70-0.82 ; 95 \%$ IC) y en mujeres $(\mathrm{RR}=0.73 ; 0.68-0.78 ; 95 \%$ IC $)$. Otro estudio realizado por WendelVos (2004), determinó que altas intensidades de actividad física en el trabajo tienen un efecto protector contra accidentes cerebrovasculares, en comparación con intensidades bajas de actividad física ( $\mathrm{RR}=0.57 ; 0.43-0.77 ; 95 \%$ IC), así como también las intensidades altas de actividad física en el tiempo libre proveen un efecto protector contra accidentes cerebrovasculares, en relación con intensidades bajas $(R R=0.79 ; 0.69$ 0.91; 95\% IC). Por último, en el meta-análisis realizado por Blair (2003), se observó que en estudios de cohortes, los individuos que realizaban actividad física con intensidades altas obtenían un efecto protector $(\mathrm{RR}=0.75 ; 0.69-0.82 ; 95 \%$ IC), así como también en estudios de casos y controles ( $R R=0.36 ; 0.25-0.52 ; 95 \%$ IC), frente a accidentes cerebrovasculares isquémicos y hemorrágicos en comparación con personas que la realizaban con intensidades bajas. 


\section{Conclusión}

De acuerdo a los estudios citados anteriormente (Li, 2012; Wendel-Vos, 2004; Blair, 2003) y al tamaño del efecto medio obtenido en el estudio meta-analítico sobre los niveles de actividad física Bajo vs. Moderado, se verifica que los resultados obtenidos son congruentes y similares. En función de ello, se puede concluir que la práctica de actividad física en forma moderada ofrece un efecto protector sobre los individuos que la realizan, disminuyendo en un $22 \%$ el riesgo de estos a padecer enfermedades cardiovasculares.

En el caso del análisis de los niveles de actividad física Moderado vs. Alto, de acuerdo al tamaño del efecto medio obtenido y comparándolo con el estudio de Wendel-Vos (2004), se puede señalar que los resultados obtenidos presentan similitud. De esta manera, se concluye que la práctica de actividad física en niveles altos ofrece un efecto protector sobre los individuos que la realizan, disminuyendo en un $19 \%$ el riesgo de estos a padecer enfermedades cardiovasculares, en relación con niveles de actividad física moderada. No obstante, cabe destacar que se detectó heterogeneidad en el TE y no pudo ser explicada por ninguna de las variables moderadoras.

Finalmente, evaluados los niveles de actividad física Bajo vs. Alto, y comparando el tamaño del efecto medio obtenido en relación con diferentes estudios (Wendel-Vos, 2004; Li, 2012; Blair, 2003), se puede concluir que intensidades altas de actividad física proveen un efecto protector en relación con intensidades bajas. En consecuencia y según los resultados obtenidos, el riesgo de padecer enfermedades cardiovasculares disminuye en un $39 \%$. 


\section{Bibliografía}

Barengo, N.C., Hu, G., Lakka, T.A., Pekkarinen, H., Nissinen, A., \& Tuomilehto, J. (2004). Low physical activity as a predictor for total and cardiovascular disease mortality in middle-aged men and women in Finland. European Heart Journal, 25 (24), 2204-2211. doi: 10.1016/j.ehj.2004.10.014.

Becker, B. J. (1988). Synthesizing standardized mean-change measures. British Journal of Mathematical and Statistical Psychology, 41 (2), 257-278. doi: 10.1111/j.2044-8317.1988.tb00901.x.

Blair, S.N., Kohl, H.R., Paffenbarger, R.S. (1989). Physical fitness and all-cause mortality: A prospective study of healthy men and women. JAMA, 262 (17), 23952401. doi:10.1001/jama.1989.03430170057028.

Carlson, K. D., \& Schmidt, F. L. (1999). Impact of experimental design on effect size: Findings from the research literature on training. Journal of Applied Psychology, 84 (6), 851-862.

Recuperado

de

http://www.management.pamplin.vt.edu/directory/Articles/Carlson5.pdf.

Cohen J. (1988). Statistical power analysis for the behavioral sciences. $2^{\mathrm{a}}$ ed. Nueva York: Academic Press.

Cooper, H. (2010) .Research synthesis and Meta-analysis: A step-by-step approach. 3ra edition. Thousand Oaks, CA: Sage.

Egger, M., Smith, G.D., Schneider, M., Minder, C. (1997). Bias in meta-analysis detect by a simple, graphical test. BMJ, 315, 629-634. Recuperado de http://www.ncbi.nlm.nih.gov/pmc/articles/PMC2127453/pdf/9310563.pdf.

Ekelund, L., Haskell, W.L., Johnson, J.L., et. al. (1988). Physical fitness as a predictor of cardiovascular mortality in asymptomatic North American men. N. Engl. J. Med, 319 (21), 1379-1384. doi: 10.1056/NEJM198811243192104.

Letón Molina, E., Pedromino Marino, A. (2001). Introducción Al Análisis de Datos en Meta-Análisis. Diaz de Santos.

Everett, B.M., Conen, D., Buring, J.E., Moorthy, M.V., Lee, I.M., \& Albert, C. (2011). Physical Activity and the Risk of Incident Atrial Fibrillation in Women. Circ. Cardiovascular Qual Outcomes, 4 (3), 321-327. doi: 10.1161/CIRCOUTCOMES.110.951442. 
Gielen, S., Schuler, G., \& Adams, V. (2010). Cardiovascular Effects of Exercise Training: Molecular Mechanisms. Circulation, 122 (12), 1221-1238. doi: 10.1161/CIRCULATIONAHA.110.939959.

Girotra, S., Kitzman, D.W., Kop, W.J., Stein, P.K., Gottdiener, J.S., \& Mukamal, K.J. (2012). Heart Rate Response to a Timed Walk \& Cardiovascular Outcomes in Older Adults: The Cardiovascular Health Study. Cardiology, 122(2), 69-75. doi: $10.1159 / 000338736$.

Harris R., J., Bradburn, M., J., Deeks, J., J., Harbord, R., M., Altman, D., G., Sterne, J., A., C. (2008). Metan: fixed- and random-effects meta-analysis. The Stata Journal 8 (1), 3-28. Recuperado de http://www2.sofi.su.se/sbc/so/files/datorovningar/Harris_et_al.pdf. Haskell W., Lee, I., Pate, R., Powell, K., Blair, S., Franklin, B., Macera, C., Heath, G., Thompson, P., \& Bauman, A. (2007). Physical Activity and Public Health Updated Recommendation for Adults From the American College of Sports Medicine and the American Heart Association. Circulation, 116 (9), 1081-1093. doi: 10.1161/CIRCULATIONAHA.107.185649.

Hedges, L. V. (1981). Distribution theory for Glass's estimator of effect size and related estimators. Journal of Educational Statistics, 6 (2), 107-128. doi: $10.3102 / 10769986006002107$.

Higgins, J. P. T. (2004). Controlling the risk of spurious findings from meta-regression. Statistics in Medicine, 23 (11), 1663-1682. dio: 10.1002/sim.1752.

Higgins, J. P. T., S. G. Thompson, J. J. Deeks, and D. G. Altman. (2003). Measuring inconsistency in meta-analyses. British Medical Journal, 327, 557-560. doi: http://dx.doi.org/10.1136/bmj.327.7414.557.

Holtermann, A., Marott, J.L., Gyntelberg, F., Søgaard, K., Suadicani, P., Mortensen, O.S., Prescott, E., \& Schnohr, P. (2013). Does the Benefit on Survival from Leisure Time Physical Activity Depend on Physical Activity at Work? A Prospective Cohort Study. PLOS ONE, 8 (1). doi:10.1371/journal.pone.0054548.

Holtermann, A., Mortensen, O.S., Burr, H., Søgaard, K., Gyntelberg, F., \& Suadicani, P. (2009). The interplay between physical activity at work and during leisure time - risk of ischemic heart disease and all-cause mortality in middle-aged Caucasian men. Scand. J. Work Environ Health, 35 (6), 466-474. doi:10.5271/sjweh.1357.

Jette, M., Sidney, K., Quenneville, J., Landry, F. (1992). Relation between cardiorespiratory fitness and selected risk factors for coronary heart disease in a population of Canadian men and women. Canadian Medican Association Journal, 146 
http://www.ncbi.nlm.nih.gov/pmc/articles/PMC1488567/pdf/cmaj00297-0097.pdf.

Kemi, O.J., Haram, P.M., Loennechen, J.P., Osnes, J.B., Skomedal, T., Wisløff, U., Ellingsen, O. (2005). Moderate vs. high exercise intensity: Differential effects on aerobic fitness, cardiomyocyte contractility, and endothelial function. Cardiovascular Research 67(1), 161 - 172. doi:10.1016/j.cardiores.2005.03.010.

Kettel Khan, L., Sobush, K., Keener, D., Goodman, K., Lowry, A., Kakietek, J., Zaro, S. (2011). Recommended Community Strategies and Measurements to Prevent Obesity in the United States. CDC, Recommended and Reports, 58 (RR07), 1-26. Recuperado de http://www.cdc.gov/MMWR/preview/mmwrhtml/rr5807al.htm.

Khaw, K.T., Jakes, R., Bingham, S., Welch, A., Luben, R., Day, N., \& Wareham, N. (2006). Work and leisure time physical activity assessed using a simple, pragmatic, validated questionnaire and incident cardiovascular disease and all-cause mortality in men and women: The European Prospective Investigation into Cancer in Norfolk prospective population study. International Journal of Epidemiology, 35 (4), 10341043. doi:10.1093/ije/dyl079.

Lee, C.D., Folsom, A.R. \& Blair, S.N. (2003). Physical Activity and Stroke Risk: A Meta-Analysis. $\quad$ Stroke, $34 \quad$ (10), 2475-2481, doi: 10.1161/01.STR.0000091843.02517.9D.

Lee, I.M., Sesso, H.D., Oguma, Y., Paffenbarger, R.S. (2003). Relative intensity of physical activity and risk of coronary heart disease. Circulation, 107 (8), $1110-1116$. doi: 10.1161/01.CIR.0000052626.63602.58.

Lee, I.M., Hennekens, C.H., Berger, K., Buring J.E., \& Manson, J.E. (1999). Exercise and Risk of Stroke in Male Physicians. Stroke, 30 (1), 1-6. doi: 10.1161/01.STR.30.1.1. Leon, A.S., Connett, J., Jacobs, D.R., et. al. (1987). Leisure-time physical activity levels and risk of coronary heart disease and death. The Multiple Risk Factor Intervention Trial. JAMA, 258 (17), 2388-2395. doi:10.1001/jama.1987.03400170074026.

Li, A., Siegrist, J. (2012). Physical Activity and Risk of Cardiovascular Disease-A

Meta-Analysis of Prospective Cohort Studies. Int. J. Environ. Res. Public Health, 9 (2), 391-407. doi:10.3390/ijerph9020391.

Lipsey, M.W. \& Wilson, D.B. (2001). Practical meta-analysis. Thousand Oaks. CA: Sage.

López Chicarro, J., Fernández Vaquero, A. (2006). Fisiología del Ejercicio. 3ra Edición. Editorial Médica Panamericana. Madrid. 
Manson, J.E., Hu, F.B., Rich-Edwards, J.W., Colditz, G.A., Stampfer M.J., Willet W.C., Speizer, F.E., \& Hennekens, C.H. (1999). A Prospective Study Of Walking As

Compared With Vigorous Exercise In The Prevention Of Coronary Heart Disease In

Women. The New England Journal of Medicine, 341 (9), 650-658. doi:

10.1056/NEJM199908263410904.

Marín Martínez, F., Sánchez Meca, J. \& López, J. A. (2009). El meta-análisis en el ámbito de las Ciencias de la Salud: una metodología imprescindible para la eficiente acumulación del conocimiento. Fisioterapia, 31, 107-114. Recuperado de http://www.um.es/metaanalysis/pdf/5016.pdf.

McMurray, R.G., Ainsworth, B.E., Harrell, J.S., Griggs, T.R., Williams, O.D. (1998). Is physical activity or aerobic power more influential on reducing cardiovascular disease risk factors?. Medicine \& Science in Sports \& Exercise, $30 \quad$ (10), 1521-1529. doi: 10.1097/00005768-199810000-0000.

Morris, J.N., Clayton, D.G., Everitt, M.G., Semmence, A.M., \& Burgess E.H. (1989). Exercise in leisure time: coronary attack and death rates. Br Heart J, 63 (6), 325-334. doi:10.1136/hrt.63.6.325.

Morris, J.N., Everitt, M.G., Pollard, R. (1980). Vigorous exercise in leisure-time: protection against coronary heart disease. Lancet, 2 (8206), 1207-1210. doi:10.1016/S0140-6736(80)92476-9.

Morris, S. B. (2003). Estimating Effect Size from the Pretest-Posttest-Control Design. Paper presented at the 18th annual conference of the Society for Industrial and Organizational Psychology, Orlando, FL. doi:10.1177/1094428106291059.

Morris, S. B., \& DeShon, R. P. (2002). Combining effect size estimates in metaanalysis with repeated measures and independent-groups designs. Psychological Methods, 7 (1), 105-125. Recuperado de http://psych.colorado.edu/ willcutt/pdfs/morris_2002.pdf.

Naylor, C.D. (1997). Meta-analysis and the meta-epidemiology of clinical research. BMJ, 315 (7109), 617-619. Recuperado de http://www.ncbi.nlm.nih.gov/pmc/articles/PMC2127435/pdf/9310553.pdf.

Paffenbarger, R.S., Hyde, R.T., Wing, A.L. (1986). Physical activity, all-cause mortality, and longevity of college alumni. New England Journal Medicine, 314 (10), 605-613. doi: 10.1056/NEJM198603063141003.

Paffenbarger, R.S., Hyde, R.T., Wing, A.L. (1990). Physical activity and physical fitness as determinants of health and longevity. In Bouchard C, Shephard RJ, Stephens 
T et al (eds): Exercise, Fitness, and Health: a Consensus of Current Knowledge, Human Kinetics, Champaign, Ill, 33-48.

Park, S., Lee, J., Kang, D.Y., Rhee, C.W., \& Park, B.J. (2012). Indoor Physical Activity Reduces All-Cause and Cardiovascular Disease Mortality Among Elderly Women. Journal of Preventive Medicine and Public Health, 45 (1), 21-28. doi: 10.3961/jpmph.2012.45.1.21.

Powell, K.E., Thompson, P.D., Caspersen, C.J., et. al. (1987). Physical activity and the incidence of coronary heart disease. Annu Rev Public Health, 8, 253-287. doi: 10.1146/annurev.pu.08.050187.001345

Rist, P.M., Lee, I.M., Kase, C.S., Gaziano, J.M., \& Kurth, T. (2011). Physical Activity and Functional Outcomes from Cerebral Vascular Events in Men. Stroke, 42 (12), 3352-3356. doi:10.1161/STROKEAHA.111.619544.

Sackett, D, et. al. (1991). Clinical Epidemiology: A basic science for clinical medicine 2nd ed. Little, Brown \& Company.

Sánchez Meca, J. Botella, J. (2010). Revisiones sistemáticas y meta-análisis: herramientas para la práctica profesional. Papeles del Psicólogo, 31 (1), 7-17. Recuperado de http://www.um.es/metaanalysis/pdf/5029.pdf.

Sánchez-Meca, J., Marín-Martínez, F., \& Huedo-Medina, T. (2006). Modelo de efectos fijos y modelo de efectos aleatorios [Fixed-effects and random-effects models]. In J.L.R. Martín, A. Tobías, \& T. Seoane (Coords.), Revisiones Sistemáticas en Ciencias de la Vida. Toledo: FISCAM.

Segunda Encuesta Nacional de Factores de Riesgo para Enfermedades No Transmisibles. (2011). Primera Edición. Buenos Aires. Ministerio de Salud de la Nación.

Sesso, D.H., Paffenbarger, R.S., Ha, T., \& Lee, I.M. (1999). Physical Activity and Cardiovascular Disease Risk in Middle-aged and Older Women. American Journal of Epidemiology, 150 (4), 408-416. Recuperado de http://aje.oxfordjournals.org/.

Shibata, Y., Hayasaka, S., Yamada, T., Goto, Y., Ojima, T., Ishikawa, S., Kayaba, K., Gotoh, T., \& Nakamura, Y. (2010). Physical Activity and Cardiovascular Disease in Japan: The Jichi Medical School Cohort Study. J. Epidemiology, 20 (3), 225-230. doi: 10.2188/jea.JE20090051.

Shibata, Y., Hayasaka, S., Yamada, T., Ojima, T., Ishikawa, S., Kayaba, K., Gotoh, T., \& Nakamura, T. (2011). Physical Activity and Risk of Fatal or Non-Fatal 
Cardiovascular Disease Among CVD Survivors. Circ. J., 75 (6), 1368 - 1372. doi: 10.1253/circj.CJ-10-0970.

Siscovick, D.S., Weiss, N.S., Hallstrom, A.P., et. al. (1982). Physical activity and $\begin{array}{lllll}\text { primary cardiac } & \text { arrest. JAMA, } 248 \quad \text { (23), }\end{array}$ doi:10.1001/jama.1982.03330230025025.

Siscovick, D.S. (1990). Risks of exercising: sudden cardiac death and injuries. In Bouchard C, Shephard RJ, Stephens T et al (eds): Exercise, Fitness, and Health: a Consensus of Current Knowledge, Human Kinetics, Champaign, Ill, 707-713.

Siscovick, D.S., LaPorte, R.E., Newman, J.M. (1985). The disease-specific benefits and risks of physical activity and exercise. Public Health Report, 100 (2), 180-188. Recuperado de http://www.ncbi.nlm.nih.gov/pmc/articles/PMC1424738/pdf/pubhealthrep001000070.pdf.

Skerrett, P., J. \& Manson, J. (2002). Reduction in risk of coronary heart disease and diabetes. In Handbook of exercise in Diabetes. Edited by Ruderman, N., Devlin, J., T., Schneider, S., H., Kriska, A., Alexandria, V., A. American Diabetes Association, $155-$ 182.

Slattery, M.L. \& Jacobs, D.R. (1988). Physical fitness and cardiovascular disease mortality. The US railroad study, Am J Epidemiol, 127 (3), 571-580.

Sobolski, J., Kornitzer, M., De Backer, G., et. al. (1987). Protection against ischemic heart disease in the Belgian physical fitness study: Physical fitness rather than physical activity? Am J Epidemiol, 125 (4), 601-610.

Sofi, F., Capalbo, A., Cesari, F., Abbate, R., Gensini, G.F. (2008). Physical activity during leisure time and primary prevention of coronary heart disease: An updated metaanalysis of cohort studies. Eur. J. Cardiovasc. Prev. Rehabil. 15 (3), 247-257. doi: 10.1097/HJR.0b013e3282f232ac.

Spina, R.J., Ogawa, T., Martin, W.H., Coggan, A.R., Holloszy, J.O. \& Ehsani, A. A. (1992). Exercise training prevents decline in stroke volume during exercise in young healthy subjects. Journal of applied physiology, 72 (6), 2458-2462.

Sport for All - Physical Activity and the Prevention of Disease. (2007). Council of Europe, Strasbourg, France.

Swain, D.P. \& Franklin, B.A. (2006). Comparison of Cardioprotective Benefits of Vigorous Versus Moderate Intensity Aerobic Exercise. Am J Cardiol, 97, 141-147. Recuperado de https://www.sakr.ch/DOCS_PUBLIC/document_07.pdf. 
Tanasescu, M., Leitzmann, M.F., Rimm, E.B., \& Hu, F.B. (2003). Physical Activity in Relation to Cardiovascular Disease and Total Mortality Among Men With Type 2 Diabetes. Circulation, 107 (19), 2435-2439. doi: 10.1161/01.CIR.0000066906.11109.1F.

Urrútia, G. \& Bonfill, X. Declaración PRISMA: una propuesta para mejorarla publicación de revisiones sistemáticas y meta-análisis. Med Clin (Barc), 135 (11), 507511. Recuperado de http://www.prismastatement.org/PRISMA\%20Spanish\%20Sept\%202010.pdf.

Vella, C. A. \& Robergs, R. A. (2005). A review of the stroke volume response to upright exercise in healthy subjects. Br J Sports Med, 39 (9), 190-195. doi:10.1136/bjsm.2004.013037.

Wagner, A., Simon, C., Evans, A., Ferrières, J., Montaye, M., Ducimetière, P., \& Arveiler, D. (2002). Physical Activity and Coronary Event Incidence in Northern Ireland and France: The Prospective Epidemiological Study of Myocardial Infarction (PRIME). Circulation, 105 (19), 2247-2252. doi: 10.1161/ 01.CIR.0000016345.58696.4F.

Wannamethee, G., Shaper, A.G. (1992). Physical activity and stroke in British middle aged men. BMJ, 304 (6827), 597-601. doi: 10.1136/bmj.304.6827.597.

Wannamethee, S.G., Shaper, A.G., \& Walker, M. (2000). Physical Activity and Mortality in Older Men With Diagnosed Coronary Heart Disease. Circulation, 102 (12), 1358-1363. doi: 10.1161/01.CIR.102.12.1358.

Wendel-Vos, G.C.W., Schuit, A.J., Feskens, E.J.M., Boshuizen, H.C., Verschuren, W.M.M., Saris, W.H.M. \& Kromhout, D. (2004). Physical activity and stroke. A metaanalysis of observational data. International Journal of Epidemiology, 33 (4), 787-798. doi:10.1093/ije/dyh168.

Wilmore, J.H., Costill, D.L. (2004). Fisiología del Esfuerzo y del Deporte. 5ta Edición. Editorial Paidotribo.

Yu, S., Yarnell, J.W.G., Sweetnam, P.M., \& Murray, L. (2003). What level of physical activity protects against premature cardiovascular death? The Caerphilly study. Heart, 89 (5), 502-506. doi:10.1136/heart.89.5.502.

Yusuf, S., Reddy, S., Ounpuu, S. \& Anand, S. (2001). Global burden of cardiovascular diseases: Part I: General considerations, the epidemiologic transition, risk factors, and impact of urbanization. Circulation, 104, 2746-2753. doi: 10.1161/hc4601.099487. 
Zhou, A., Obuchowski, N. \& McClish D. (2002). Issues in meta-analysis for diagnostic tests. In: Zhou A, Obuchowski N, McClish D, eds. Statistical methods in diagnostic medicine. New York: Wiley \& Sons, Inc, 222-240. 


\section{ANEXO I}

\section{Resultados de Odds Ratio:}

Tabla 42. Resultado de Odds Ratio nivel Bajo vs. Moderado

\begin{tabular}{|c|c|c|c|c|c|}
\hline Study & I & OR & [95\% Conf. & Interval] & \% Weight \\
\hline Manson (a) (1999) & 1 & 0.786 & 0.633 & 0.976 & 5.78 \\
\hline Holtermann (a) (2013 & I & 0.948 & 0.874 & 1.027 & 6.98 \\
\hline I-Min Lee (1998) & I & 0.673 & 0.556 & 0.813 & 6.06 \\
\hline Girotra (2012) & 1 & 0.980 & 0.763 & 1.261 & 5.41 \\
\hline Shibata (a) (2010) & I & 0.713 & 0.435 & 1.168 & 3.15 \\
\hline Morris (1989) & 1 & 0.728 & 0.448 & 1.183 & 3.21 \\
\hline Park (2012) & I & 0.468 & 0.372 & 0.588 & 5.65 \\
\hline Barengo (2004) & I & 0.738 & 0.676 & 0.805 & 6.93 \\
\hline Sesso (1999) & I & 0.970 & 0.677 & 1.389 & 4.29 \\
\hline Wagner (2002) & I & 0.750 & 0.568 & 0.989 & 5.13 \\
\hline Rist (2011) & 1 & 0.863 & 0.734 & 1.015 & 6.34 \\
\hline Wannamethee (a) $(200$ & I & 0.667 & 0.523 & 0.852 & 5.48 \\
\hline Shibata (b) (2011) & I & 0.603 & 0.348 & 1.045 & 2.78 \\
\hline Wannamethee (b) (199 & 1 & 0.610 & 0.418 & 0.890 & 4.11 \\
\hline Everett (2011) & I & 0.857 & 0.702 & 1.046 & 5.96 \\
\hline Tanasescu (2003) & I & 0.827 & 0.586 & 1.166 & 4.44 \\
\hline Yu (2003) & 1 & 0.717 & 0.514 & 0.999 & 4.56 \\
\hline Khaw (2006) & 1 & 0.594 & 0.546 & 0.646 & 6.96 \\
\hline Holtermann (b) (2009 & I & 1.112 & 0.995 & 1.242 & 6.78 \\
\hline$D+L$ pooled $O R$ & I & 0.762 & 0.678 & 0.857 & 100.00 \\
\hline
\end{tabular}

\footnotetext{
Heterogeneity chi-squared $=137.66(\mathrm{~d} . \mathrm{f} .=18) \mathrm{p}=0.000$

I-squared (variation in OR attributable to heterogeneity) $=\mathbf{8 6 . 9}$

Estimate of between-study variance Tau-squared $=0.0493$
}

Test of $O R=1: z=4.55 p=0.000$ 


\section{Figura 15. Forest Plot de Odds Ratio Bajo vs. Moderado}

\section{Bajo vs. Moderado}

\begin{tabular}{|c|c|c|}
\hline \multirow{2}{*}{$\begin{array}{l}\text { Study } \\
\text { ID }\end{array}$} & \multirow[b]{2}{*}{ OR $(95 \% \mathrm{Cl})$} & $\%$ \\
\hline & & Weight \\
\hline Manson (a) (1999) & $0.79(0.63,0.98)$ & 5.78 \\
\hline Holtermann (a) (2013) & $0.95(0.87,1.03)$ & 6.98 \\
\hline I-Min Lee (1998) & $0.67(0.56,0.81)$ & 6.06 \\
\hline Girotra (2012) & $0.98(0.76,1.26)$ & 5.41 \\
\hline Shibata (a) (2010) & $0.71(0.43,1.17)$ & 3.15 \\
\hline Morris (1989) & $0.73(0.45,1.18)$ & 3.21 \\
\hline Park (2012) & $0.47(0.37,0.59)$ & 5.65 \\
\hline Barengo (2004) & $0.74(0.68,0.81)$ & 6.93 \\
\hline Sesso (1999) & $0.97(0.68,1.39)$ & 4.29 \\
\hline Wagner (2002) & $0.75(0.57,0.99)$ & 5.13 \\
\hline Rist (2011) & $0.86(0.73,1.01)$ & 6.34 \\
\hline Wannamethee (a) (2000) & $0.67(0.52,0.85)$ & 5.48 \\
\hline Shibata (b) (2011) & $0.60(0.35,1.05)$ & 2.78 \\
\hline Wannamethee (b) (1992) & $0.61(0.42,0.89)$ & 4.11 \\
\hline Everett (2011) & $0.86(0.70,1.05)$ & 5.96 \\
\hline Tanasescu (2003) & $0.83(0.59,1.17)$ & 4.44 \\
\hline Yu (2003) & $0.72(0.51,1.00)$ & 4.56 \\
\hline Khaw (2006) & $0.59(0.55,0.65)$ & 6.96 \\
\hline Holtermann (b) (2009) & $1.11(1.00,1.24)$ & 6.78 \\
\hline Overall (I-squared $=86.9 \%, p=0.000)$ & $0.76(0.68,0.86)$ & 100.00 \\
\hline NOTE: Weights are from random effects analysis & & \\
\hline $\begin{array}{l}T \\
.1\end{array}$ & $\begin{array}{l}T \\
10\end{array}$ & \\
\hline
\end{tabular}

Fuente: Elaboración propia en base a estudios incluídos 
Tabla 43. Resultados de Odds Ratio nivel Moderado vs. Alto

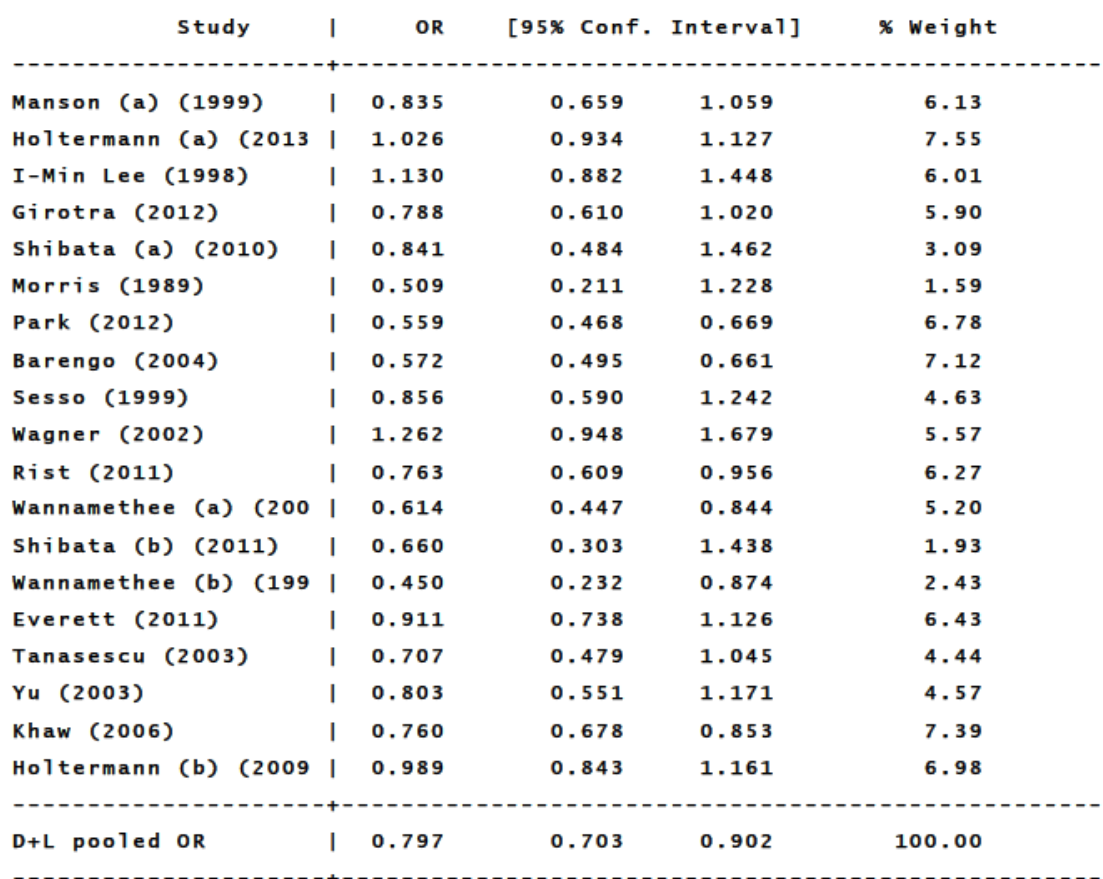

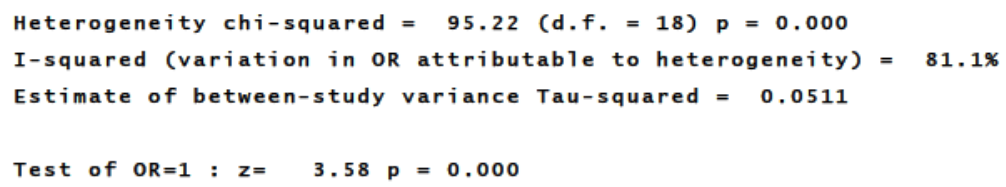


Figura 16. Forest Plot de Odds Ratio Moderado vs. Alto

\section{Moderado vs. Alto}

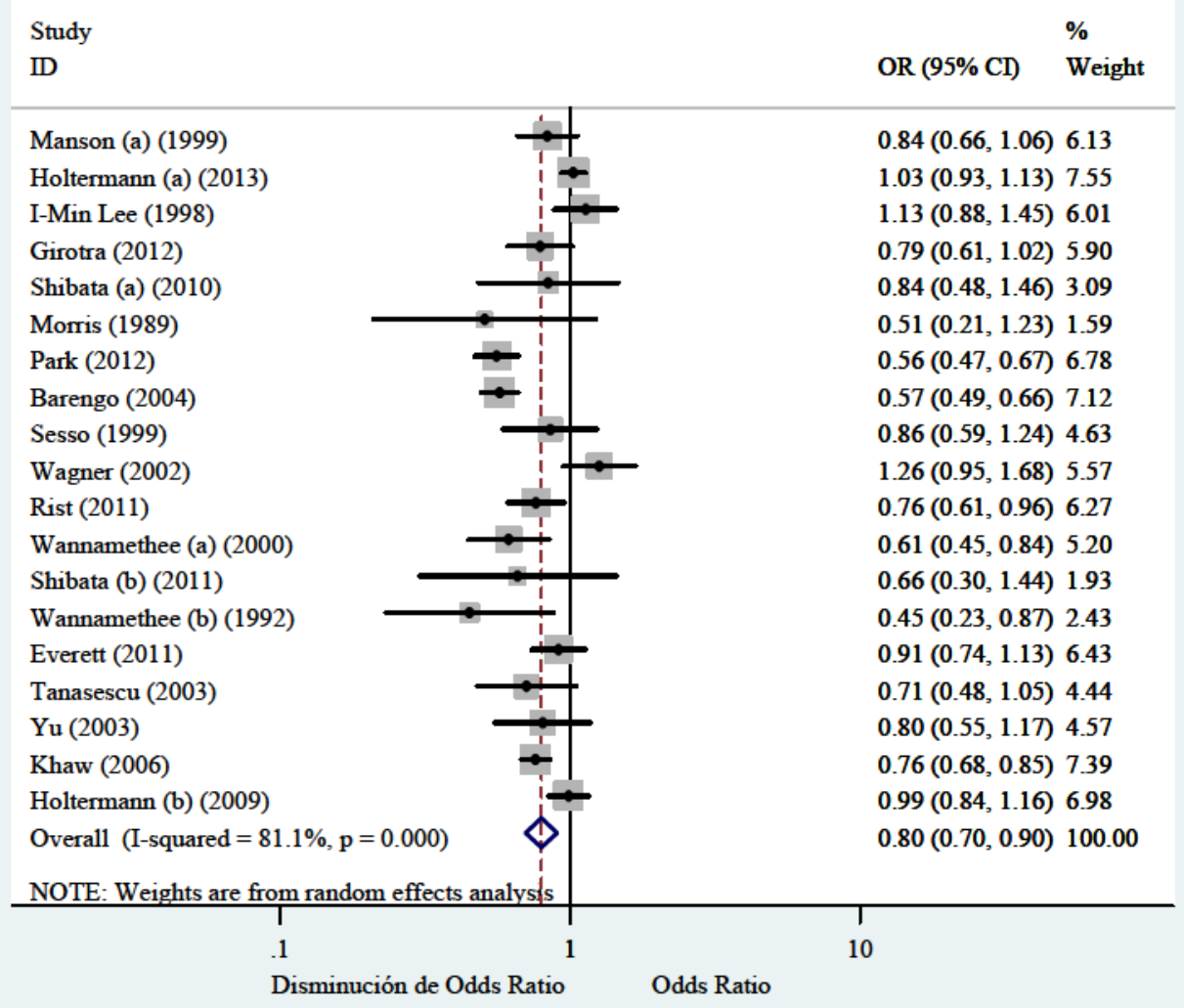

Fuente: Elaboración propia en base a estudios incluídos 
Tabla 44. Resultados de Odds Ratio nivel Bajo vs. Alto

\begin{tabular}{|c|c|c|c|c|c|}
\hline Study & I & OR & {$[95 \%$ Conf. } & Interval] & \% Weight \\
\hline Manson (a) (1999) & I & 0.656 & 0.522 & 0.826 & 5.74 \\
\hline Holtermann (a) (2013 & I & 0.972 & 0.883 & 1.071 & 6.11 \\
\hline I-Min Lee (1998) & I & 0.760 & 0.586 & 0.985 & 5.62 \\
\hline Girotra (2012) & I & 0.773 & 0.597 & 1.000 & 5.63 \\
\hline Shibata (a) (2010) & I & 0.599 & 0.358 & 1.005 & 4.41 \\
\hline Morris (1989) & I & 0.371 & 0.176 & 0.782 & 3.35 \\
\hline Park (2012) & 1 & 0.262 & 0.205 & 0.334 & 5.68 \\
\hline Barengo (2004) & I & 0.422 & 0.364 & 0.489 & 6.00 \\
\hline Sesso (1999) & I & 0.830 & 0.586 & 1.177 & 5.23 \\
\hline Wagner $(2002)$ & I & 0.946 & 0.728 & 1.230 & 5.61 \\
\hline Rist (2011) & I & 0.659 & 0.518 & 0.838 & 5.70 \\
\hline Wannamethee (a) (200 & I & 0.410 & 0.304 & 0.553 & 5.45 \\
\hline Shibata (b) (2011) & I & 0.398 & 0.195 & 0.813 & 3.49 \\
\hline Wannamethee (b) (199 & I & 0.275 & 0.145 & 0.519 & 3.83 \\
\hline Everett (2011) & I & 0.781 & 0.636 & 0.958 & 5.83 \\
\hline Tanasescu (2003) & I & 0.585 & 0.401 & 0.853 & 5.09 \\
\hline $\mathrm{Yu}(2003)$ & I & 0.576 & 0.404 & 0.821 & 5.20 \\
\hline Khaw (2006) & I & 0.452 & 0.401 & 0.509 & 6.07 \\
\hline Holtermann (b) (2009 & I & 1.100 & 0.930 & 1.302 & 5.94 \\
\hline$D+L$ pooled $O R$ & I & 0.595 & 0.486 & 0.728 & 100.00 \\
\hline
\end{tabular}

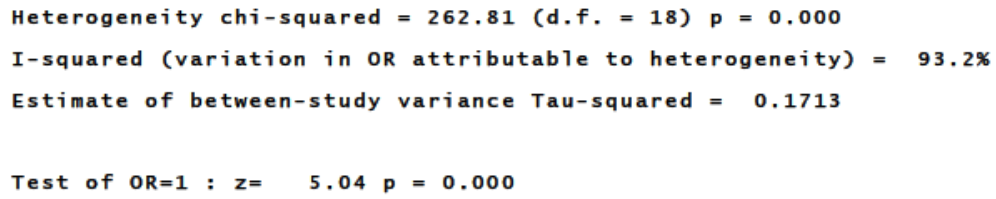


Figura 17. Forest Plot de Odds Ratio Bajo vs. Alto

\section{Bajo vs. Alto}

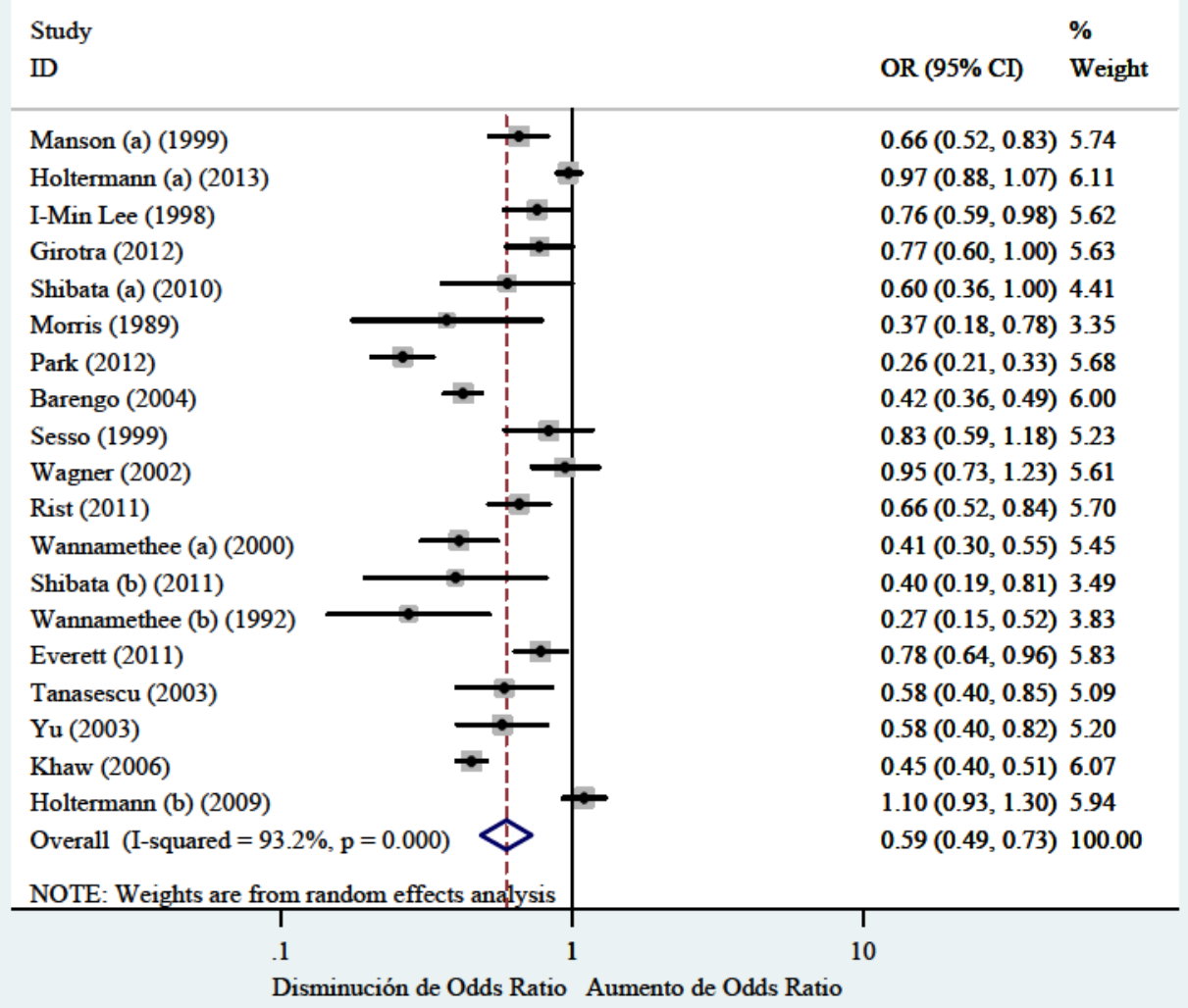

Fuente: Elaboración propia en base a estudios incluídos 
ANEXO II

Resultados de nivel Bajo vs. Moderado:

Figura 18. Meta-regresión sobre la variable porcentaje de varones

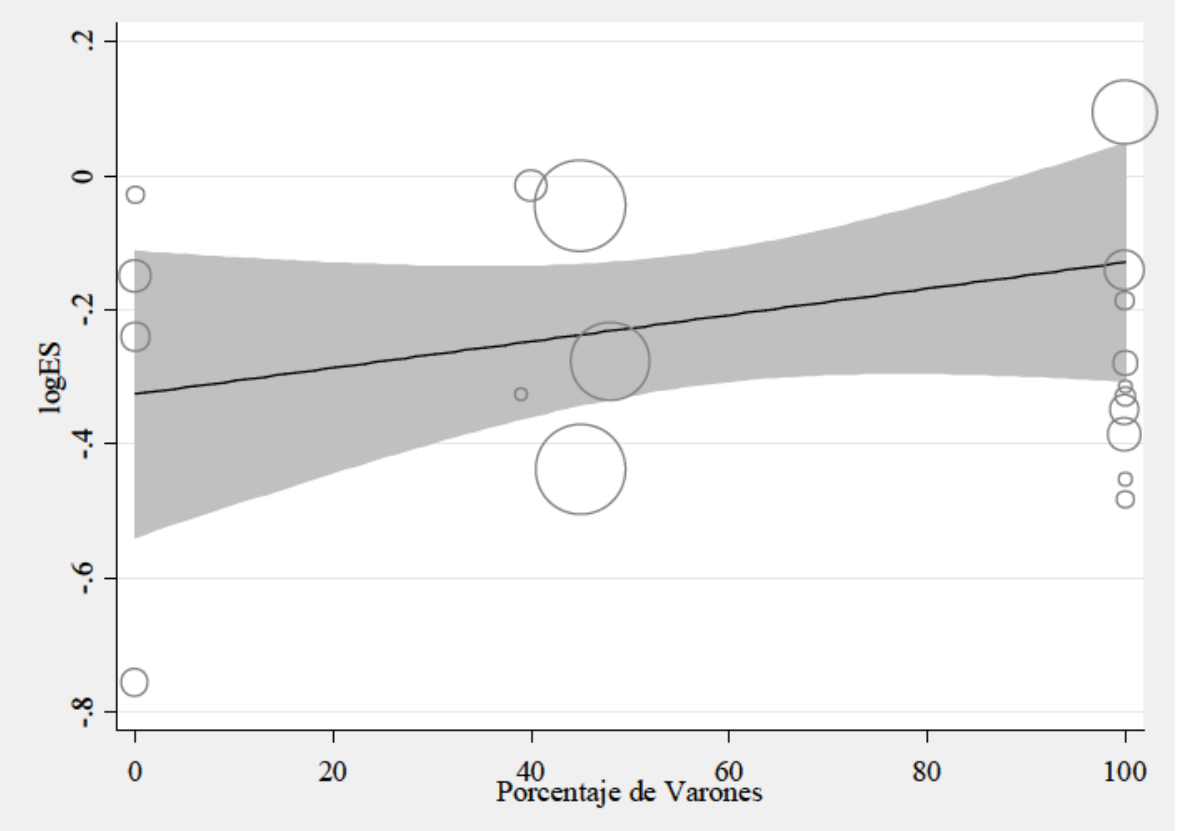

Figura 19. Meta-regresión sobre la variable edad de los participantes

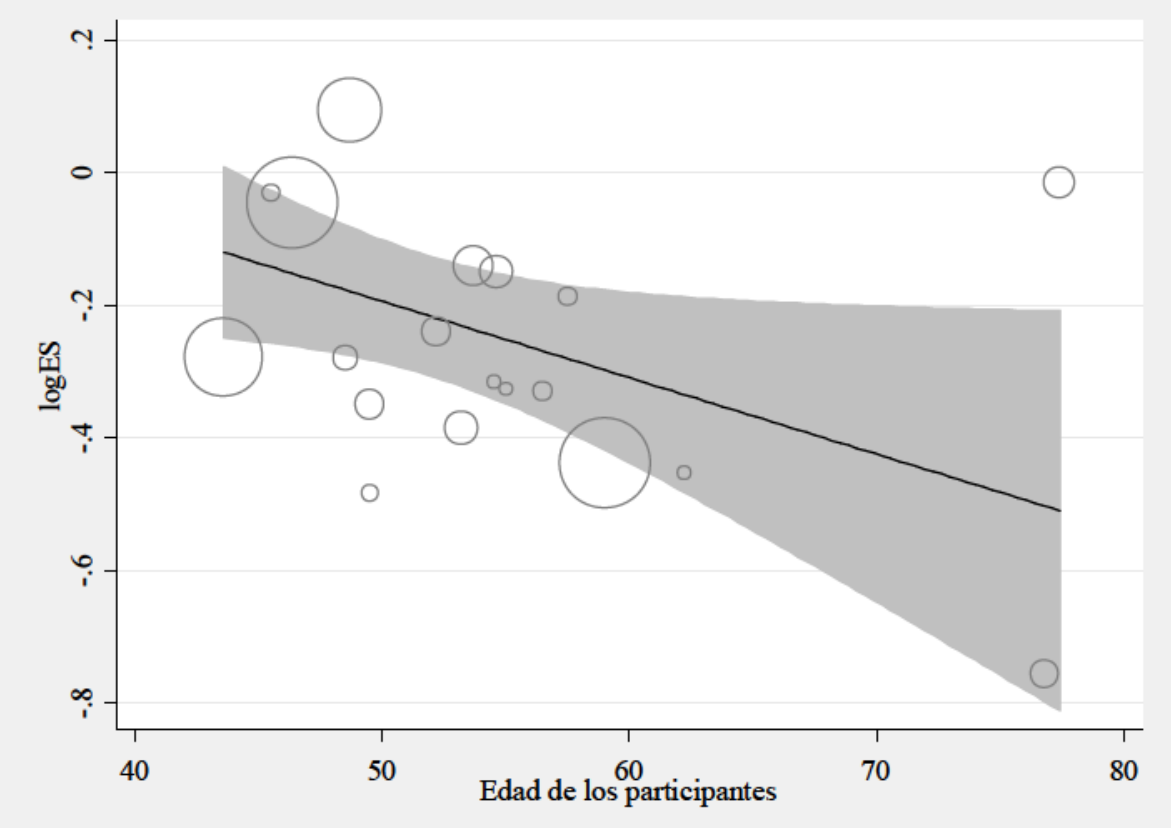


Figura 20. Meta-regresión de la variable tamaño muestral

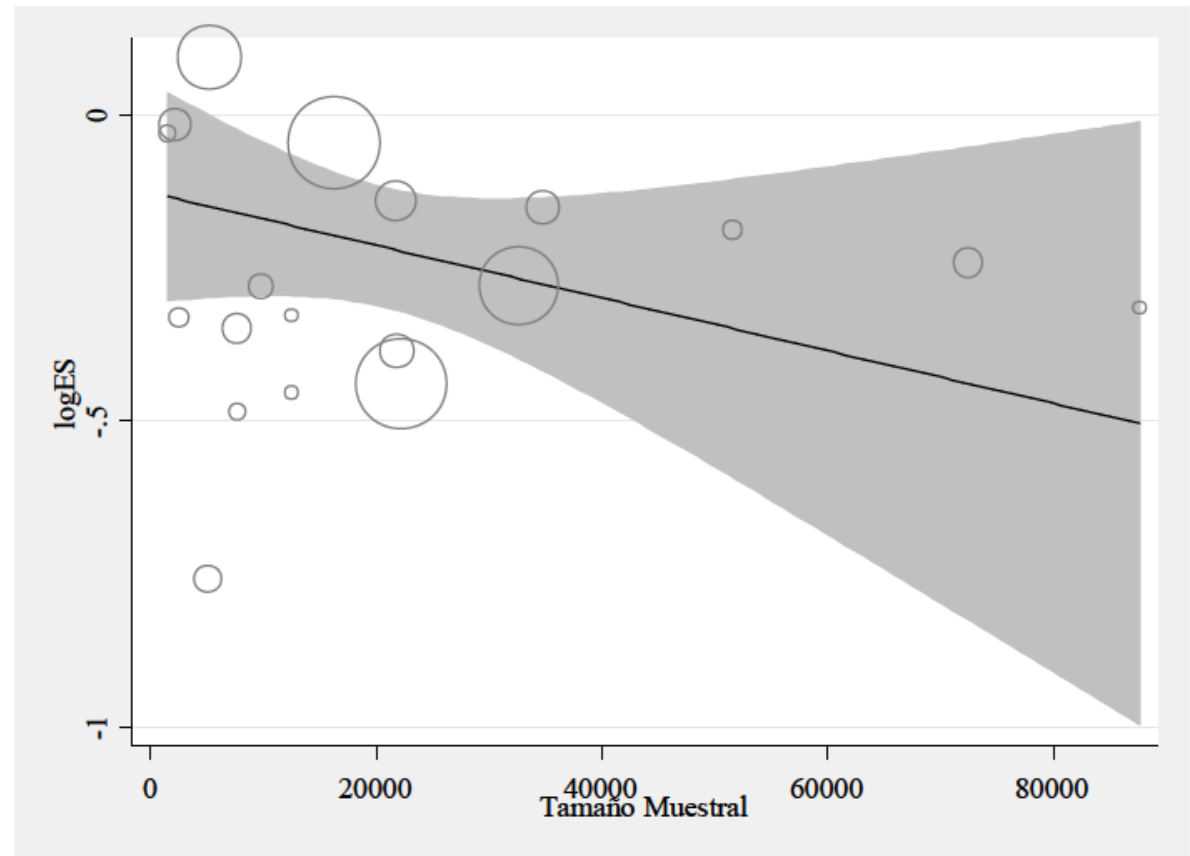

Figura 21. Meta-regresión de la variable años de seguimiento

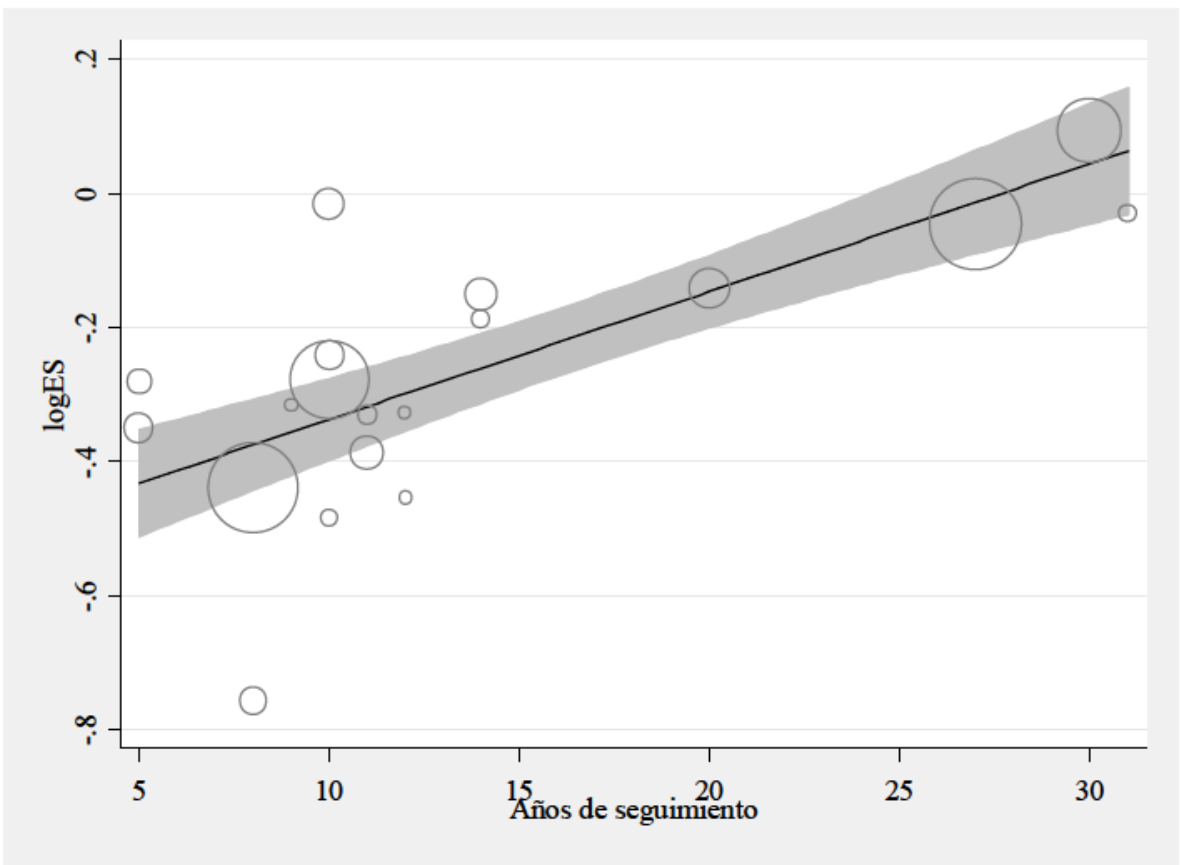


Figura 22. Meta-regresión de la variable año de publicación

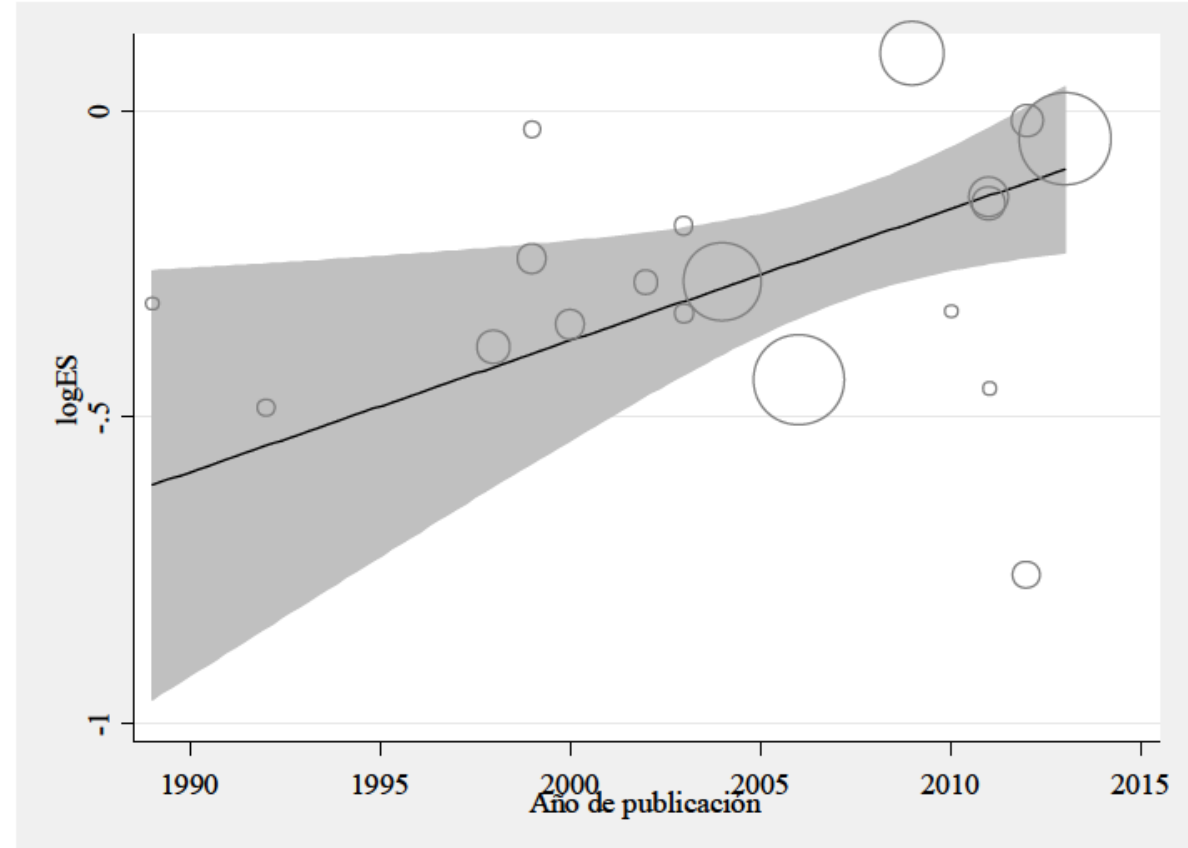

Resultados nivel Moderado vs. Alto:

Figura 23. Meta-regresión de la variable porcentaje de varones

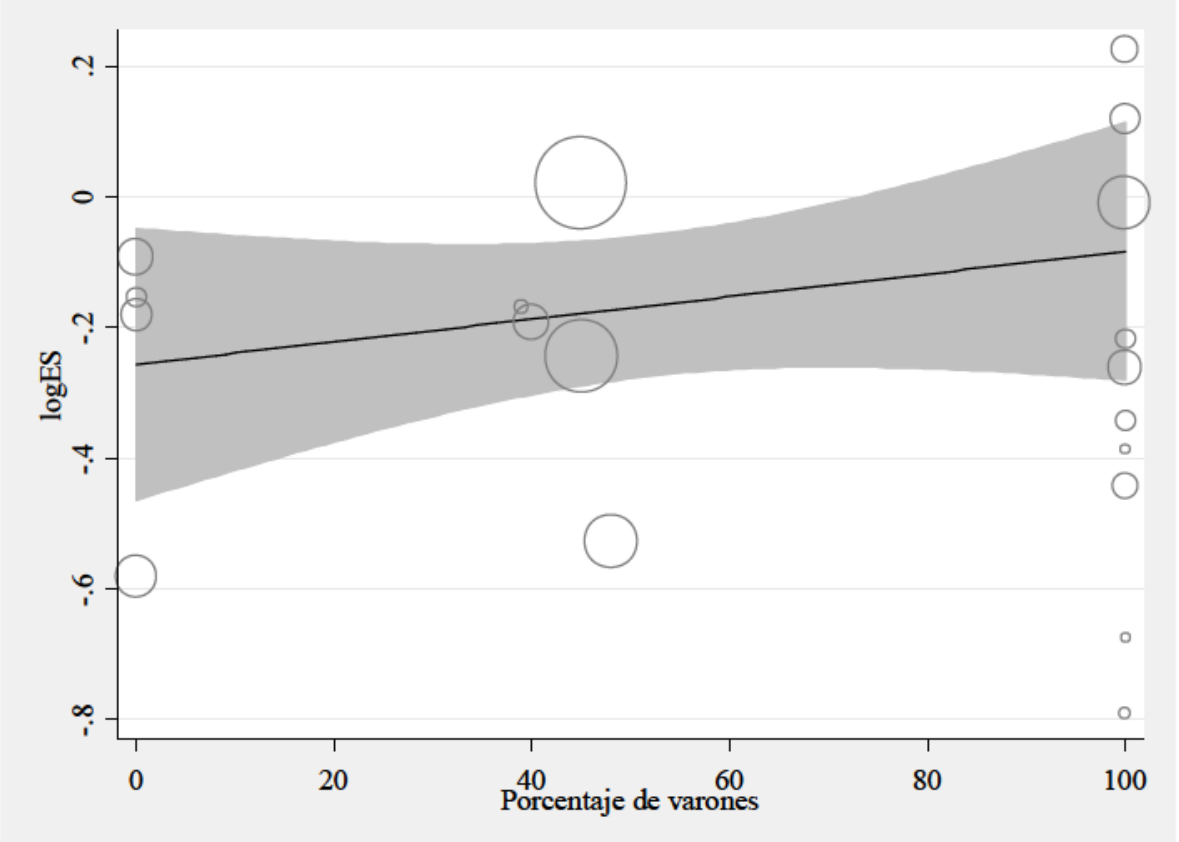


Figura 24. Meta-regresión de la variable edad media

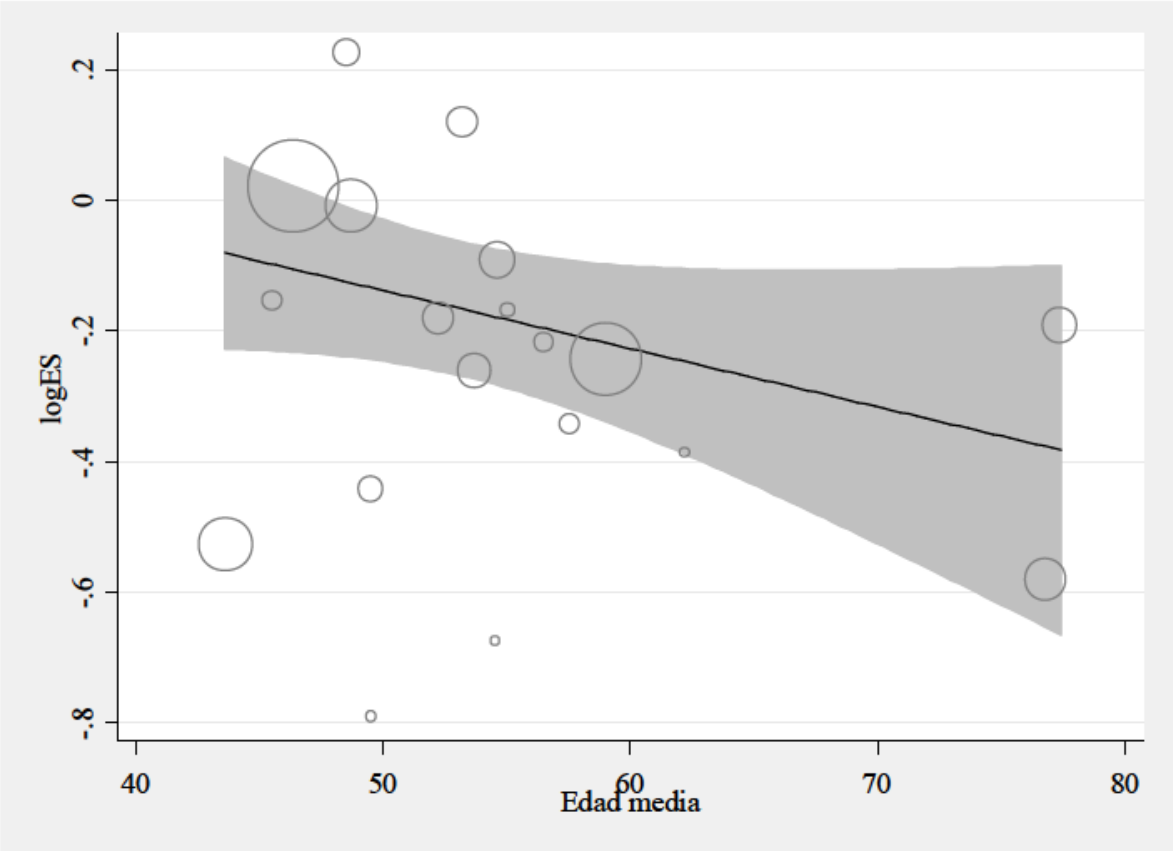

Figura 25. Meta-regresión de la variable tamaño muestral

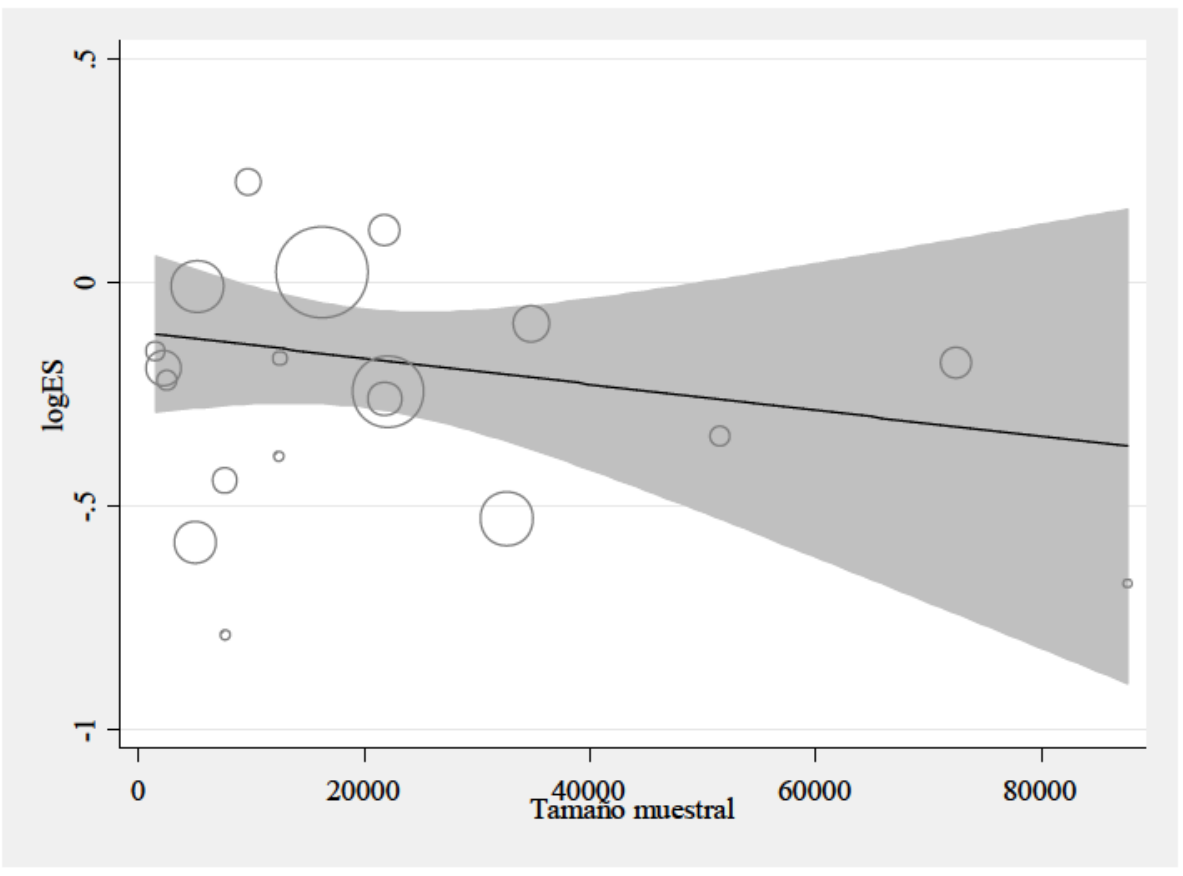


Figura 26. Meta-regresión de la variable años de seguimiento

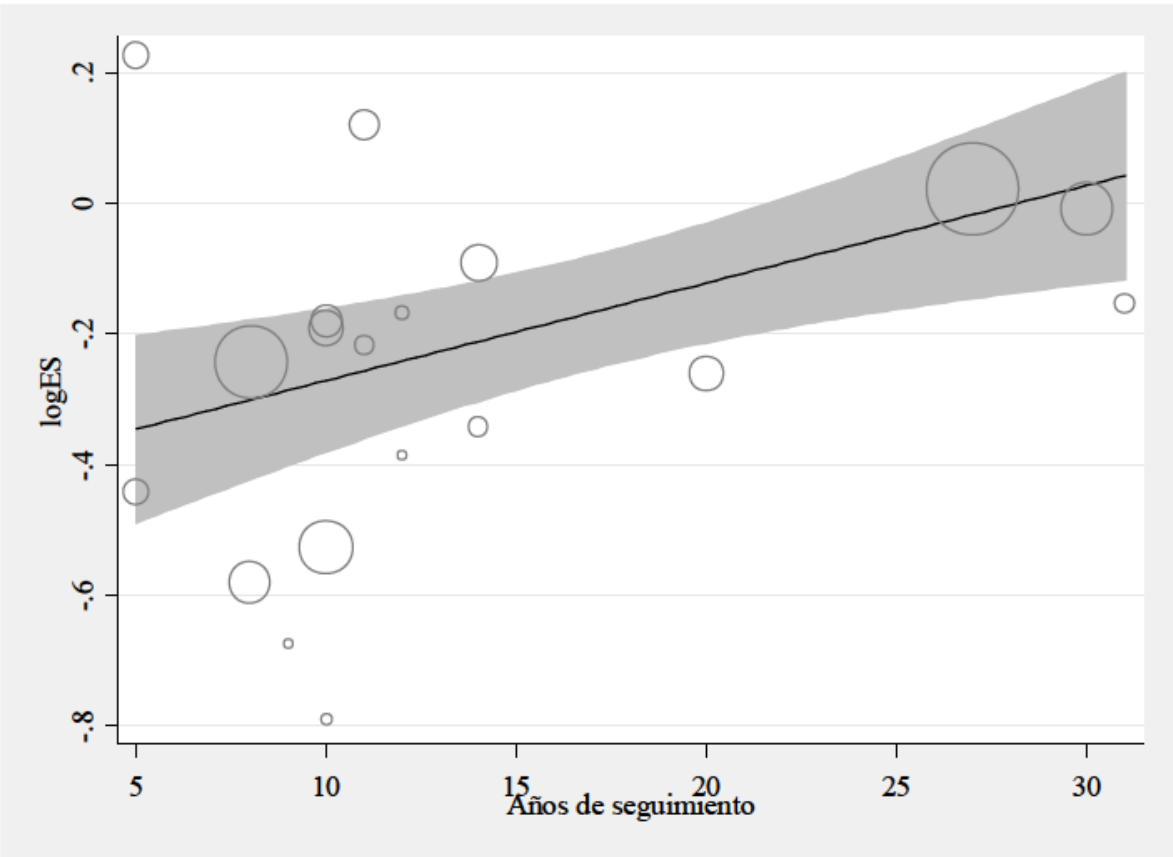

Figura 27. Meta-regresión de la variable año de publicación

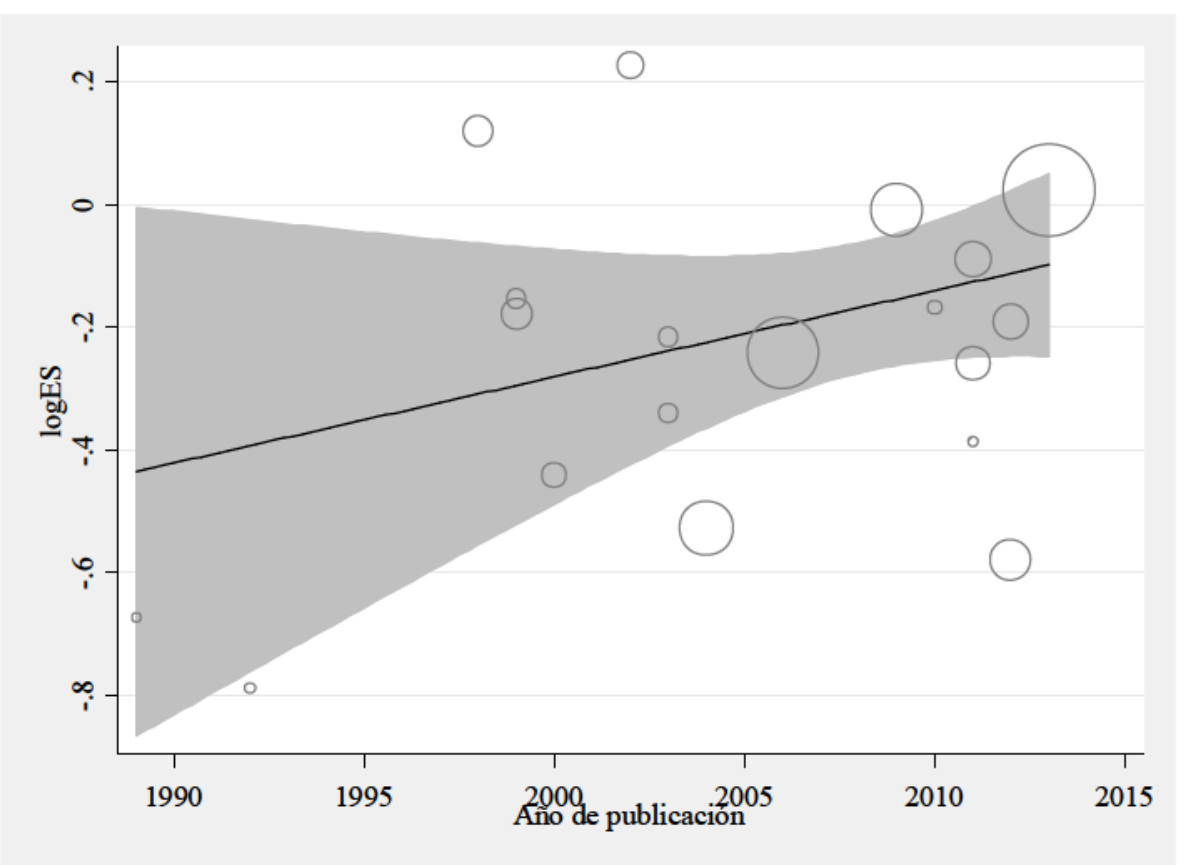


Resultados nivel Bajo vs. Alto:

Figura 28. Meta-regresión de la variable porcentaje de varones

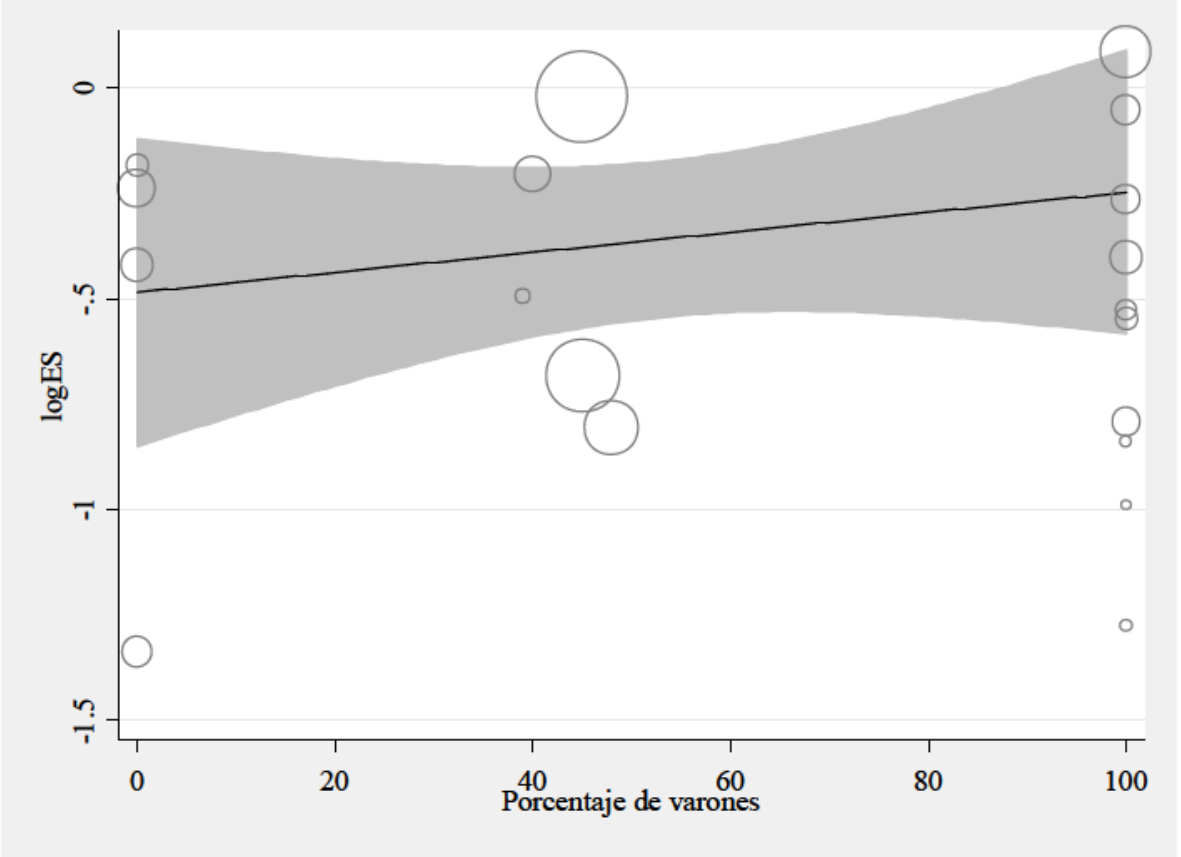

Figura 29. Meta-regresión de la variable edad media

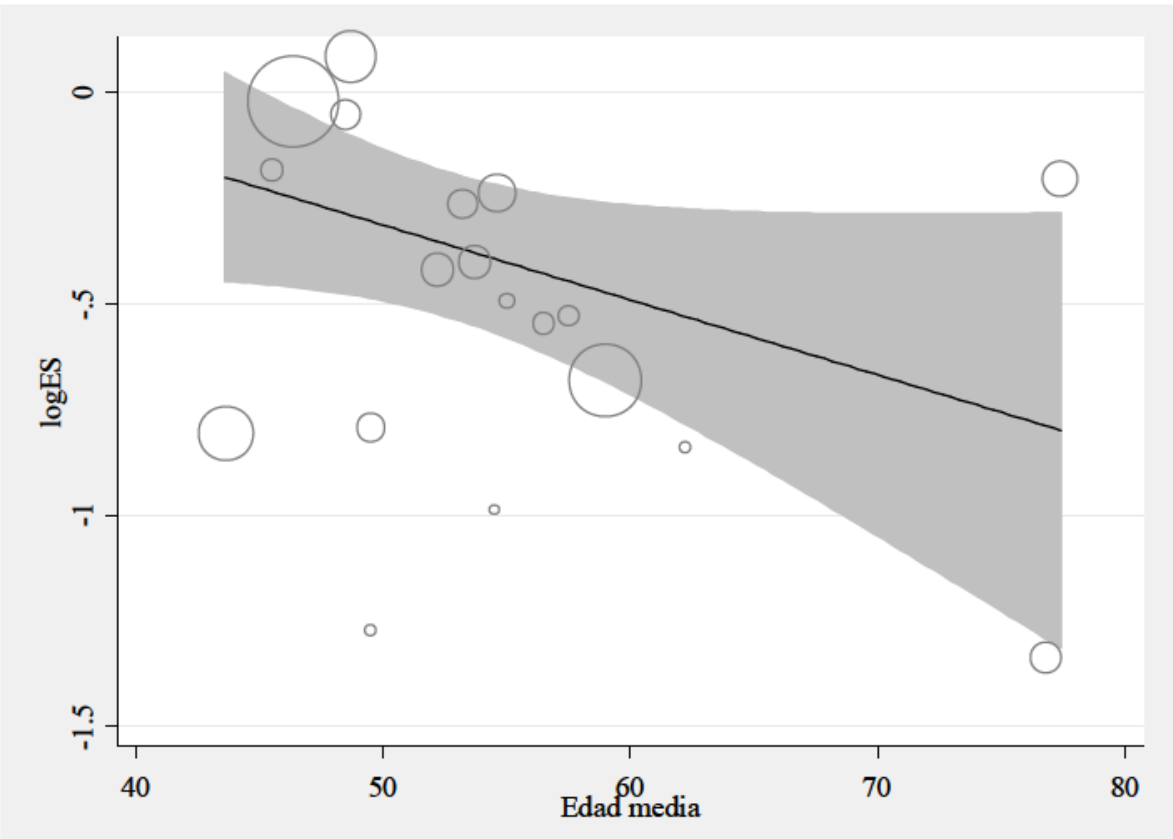


Figura 30. Meta-regresión sobre la variable tamaño muestral

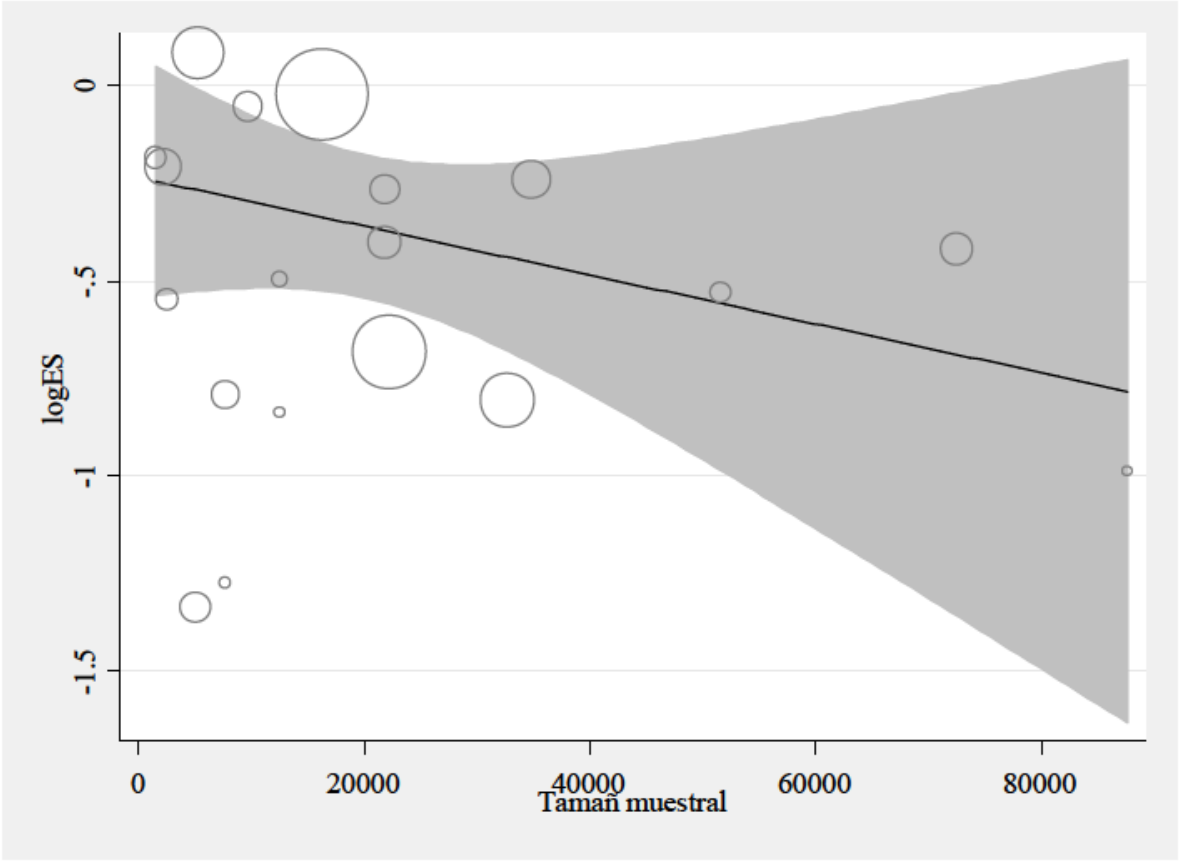

Figura 31. Meta-regresión de la variable años de seguimiento

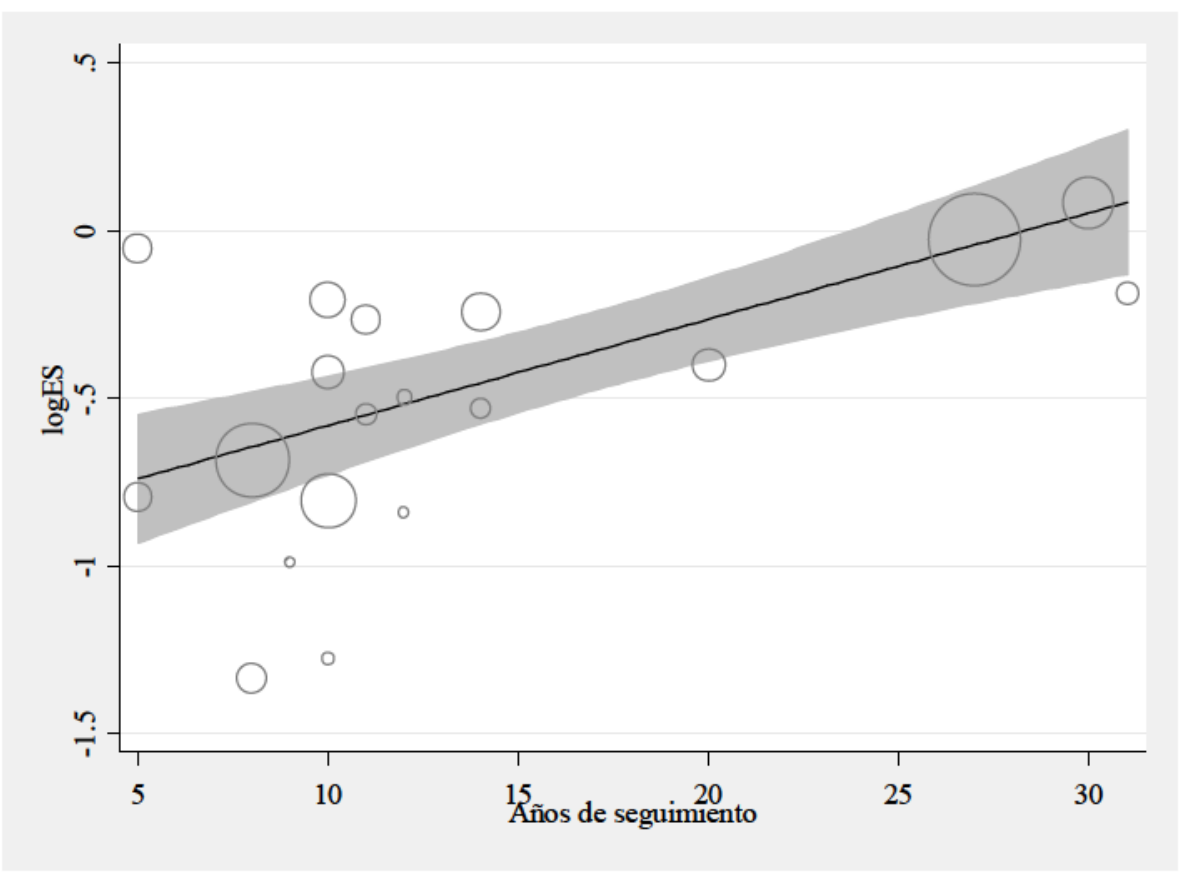


Figura 32. Meta-regresión de la variable año de publicación

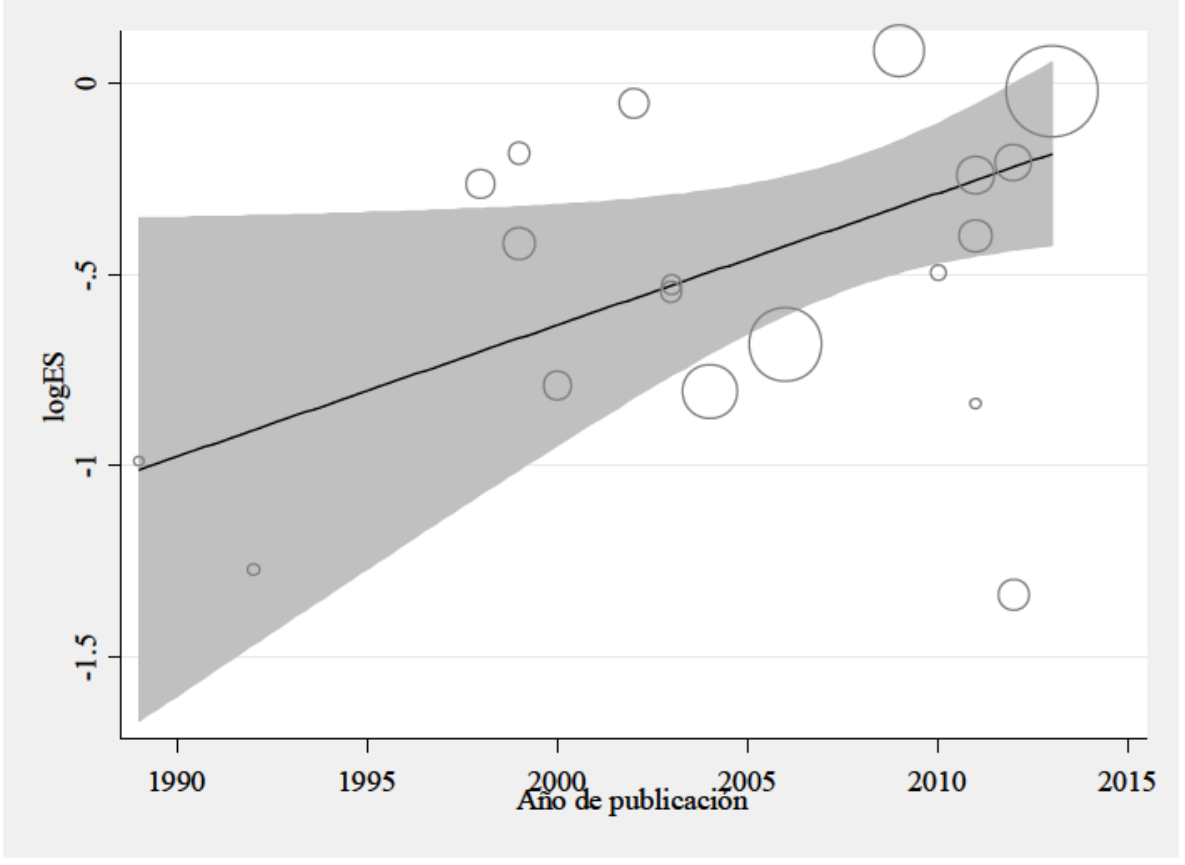




\section{ANEXO III}

\section{BÚSQUEDA DE ESTUDIOS EN PUBMED:}

\section{1. ¿Qué es PUBMED?}

PUBMED es uno de los mejores sistemas de búsqueda existentes hasta el momento referente a temas de ciencias de la salud. Es un proyecto que fue desarrollado por el NCBI (National Center for Biotechnology Information) dentro de la NLM (National Library of Medicine) puede ser consultado gratuitamente por cualquier persona que cuente con acceso a internet y una computadora.

Este sistema de búsqueda permite el acceso a bases de datos compiladas por la NLM, siendo una de las más importantes MEDLINE. Esta base de datos dispone de artículos que pueden visualizarse gratuitamente desde el resumen hasta texto completo.

\subsection{Vocabulario en MEDLINE}

MEDLINE utiliza un vocabulario controlado denominado MeSH (Medical Subject Headings). Este vocabulario controlado está compuesto por términos llamados descriptores que se organizan de manera jerárquica y están categorizados en una estructura de árbol MeSH que se actualiza anualmente. Actualmente los descriptores están estructurados de la siguiente manera:

Tabla 45. Descriptores MeSH

\begin{tabular}{|l|l|}
\hline & DESCRIPTORES \\
\hline A & Anatomía \\
\hline B & Organismos \\
\hline C & Enfermedades \\
\hline D & Químicos y Drogas \\
\hline E & $\begin{array}{l}\text { Análisis, Técnicas y Diagnóstico } \\
\text { Terapéutico y Equipamiento }\end{array}$ \\
\hline F & Psiquiatría y Psicología \\
\hline
\end{tabular}




\begin{tabular}{|l|l|}
\hline G & Fenómenos y Procesos \\
\hline H & Disciplinas y Ocupaciones \\
\hline I & $\begin{array}{l}\text { Antropología, Educación, Sociología y } \\
\text { Fenómenos Sociales }\end{array}$ \\
\hline J & Tecnología, industria, Agricultura \\
\hline K & Humanidades \\
\hline L & Ciencias de la Información \\
\hline M & Denominaciones de Grupos. \\
\hline N & Cuidado de la Salud \\
\hline V & Características de Publicaciones \\
\hline Z & Localizaciones Geográficas \\
\hline
\end{tabular}

Adaptado de http://www.nlm.nih.gov/mesh/2014/mesh_browser/MeSHtree.html 